\title{
Neolignans and Sesquiterpenes from Leaves and Embryogenic Cultures of Ocotea catharinensis (Lauraceae)
}

\author{
Mariko Funasaki, ${ }^{a}$ Ana Luísa L. Lordello, ${ }^{b}$ Ana Maria Viana, ${ }^{c}$ Claudete Santa-Catarina, ${ }^{d}$ \\ Eny I. S. Floh, ${ }^{d}$ Massayoshi Yoshida ${ }^{a, e}$ and Massuo J. Kato ${ }^{*, a}$
}

anstituto de Química, Universidade de São Paulo, CP 26077, 05513-970 São Paulo-SP, Brazil

${ }^{b}$ Departamento de Química, Universidade Federal do Paraná, CP 19081, 81531-990 Curitiba-PR, Brazil

'Departamento de Botânica, Centro de Ciências Biológicas, Universidade Federal de Santa Catarina, 88040-900 Florianópolis-SC, Brazil

${ }^{d}$ Instituto de Biociências, Universidade de São Paulo, CP 11461, 05508-900 São Paulo-SP, Brazil

${ }^{e}$ Centro de Biotecnologia da Amazônia, Av. Gov. Danilo de Matos Areosa, 690, 69075-351 Manaus-AM, Brazil

\begin{abstract}
Foram isoladas de extratos de folhas de Ocotea catharinensis Mez (Lauraceae) quatorze neolignanas sendo nove benzofurânicas (incluindo três novas substâncias 1e, $2 \mathbf{f}$ e 4b), uma secobenzofurânica inédita (3b), duas biciclo[3.2.1] octânicas (incluindo a nova 5c), dois novos dímeros biciclo[3.2.1] octânicos (7a e 7b) e ainda dois sesquiterpenos (incluindo o novo humulanol 9). Nos embriões somáticos de $O$. catharinensis foram identificadas sete neolignanas incluindo uma nova neolignana biciclo[3.2.1] octânica (4a).
\end{abstract}

The extracts from leaves of Ocotea catharinensis Mez (Lauraceae) were found to contain fourteen neolignans and two sesquiterpenes: nine benzofuran types (including three new compounds 1e, $2 \mathbf{f}$ and $\mathbf{4 b}$ ), one new seco-benzofuran type (3b), two bicyclo[3.2.1] octane types (including the new compound 5c), two new dimers of bicyclo[3.2.1] (including a new humulanol 9). In addition, seven neolignans were also showed to occur in somatic embryos of $O$. catharinensis including one new bicyclo[3.2.1] loctane type (4a).

Keywords: Ocotea catharinensis, benzofuran neolignans, bicyclo[3.2.1]octane neolignans, humulane sesquiterpene, somatic embryos

\section{Introduction}

Ocotea catharinensis (Lauraceae) is a woody plant species found in southern Atlantic forest in Brazil, which produces excellent quality of timber. The extensive logging over the past thirty years associated with difficulties for propagation has led its natural population to be significant decrease. Since $O$. catharinensis has been included as endangered species, a somatic embryogenic system was developed aiming to a massive propagation. ${ }^{1,2}$

The Ocotea has been one of the most phytochemically investigated Lauraceous genus and their major secondary compounds were showed to be phenylpropanoidderived including several sub-classes of neolignans. ${ }^{3}$ Previous phytochemical studies carried out in leaves

*e-mail: majokato@iq.usp.br of $O$. catharinensis collected at Horto Florestal (Serra da Cantareira), São Paulo State, Brazil, reported the occurrence of benzofuran $(\mathbf{1 b}, \mathbf{1 c}, \mathbf{1 d}, \mathbf{2 b}, \mathbf{2 c}, \mathbf{2 d}, \mathbf{2 e}$ and $\mathbf{2 h})$ and bicyclo[3.2.1] octane (5a, 5b and $\mathbf{5 d})$ neolignans. ${ }^{4}$ Representatives of both sub-classes of neolignans have also been previously isolated from barks and woods of a specimen collected in São Paulo State, ${ }^{5,6}$ and also from wood and leaves of $O$. porosa ("imbuia") collected in Rio Grande do Sul State, southern Brazil. ${ }^{7-9}$

This work describes the isolation and characterization of major secondary compounds from leaves collected at Vale do Itajaí, Santa Catarina State, Brazil and from embryogenic cultures developed from the same plant source. The extracts from leaves afforded seven new neolignans $\mathbf{1 e}, \mathbf{2} \mathbf{f}, \mathbf{3} \mathbf{b}$, $\mathbf{4 b}, \mathbf{5 c}, \mathbf{7 a}, \mathbf{7 b}$, besides seven previously reported ones 1a ${ }^{6,10} \mathbf{1 d},{ }^{6} \mathbf{2 a},{ }^{6,10,11} \mathbf{2 d},{ }^{4} \mathbf{2 e},{ }^{6} \mathbf{2 g},{ }^{12} \mathbf{5 e} \cdot{ }^{13}$ Additionally, a new sesquiterpene of humulane type (9), besides known 
<smiles>C=CC1(C=O)C(C)C(Br)OC2=C(OC)C(=O)[C@@H](OC)C[C@@H]21</smiles>

1a $\mathrm{Ar}=\alpha-\mathrm{Pi}, \beta-\mathrm{Me}$

1b $\mathrm{Ar}=\beta-\mathrm{Pi}, \alpha-\mathrm{Me}$

$1 \mathrm{c} \mathrm{Ar}=\beta-\mathrm{Pi}, \beta-\mathrm{Me}$

1d $\mathrm{Ar}=\beta-\mathrm{Ve}, \beta-\mathrm{Me}$

1e $\mathrm{Ar}=\alpha-\mathrm{Tp}, \beta-\mathrm{Me}$<smiles>[R]C1=CC(=O)[C@]2([R])O[C@H](Br)[C@@H](C)[C@]2(CC=C)C1</smiles>

2a $\mathrm{Ar}=\alpha-\mathrm{Pi}, \mathrm{R}^{1}=\mathrm{H}, \quad \mathrm{R}^{2}=\mathrm{H}$

2b Ar $=\beta-P i, \quad R^{1}=H, \quad R^{2}=H$

2c Ar $=\beta-P i, \quad R^{1}=H, \quad R^{2}=M e$

2d Ar $=\alpha-V e, R^{1}=H, \quad R^{2}=H$

2e $\mathrm{Ar}=\beta$-Ve, $\mathrm{R}^{1}=\mathrm{H}, \quad \mathrm{R}^{2}=\mathrm{H}$

2f $\mathrm{Ar}=\beta$-Ve, $\mathrm{R}^{1}=\mathrm{OMe}, \mathrm{R}^{2}=\mathrm{H}$

2g Ar $=\alpha-M p, R^{1}=H, \quad R^{2}=H$

2h Ar $=\beta-T p, R^{1}=H, \quad R^{2}=H$<smiles>[Y6]C(=O)[C@H](C)[C@]1(CC=C)C[C@H](OC)C=C([R])C1=O</smiles>

$3 a \mathrm{R}=\mathrm{H}$

$3 b \mathrm{R}=\mathrm{OMe}$<smiles>[R7]C1=C[C@@H](OC)C[C@@]2(CC=C)[C@@H](C)[C@@H]([AlH2])O[C@]12O</smiles>

4a $\mathrm{Ar}=\alpha-\mathrm{Pi}, \quad \mathrm{R}^{1}=\mathrm{OMe}, \mathrm{R}^{2}=\mathrm{H}$

4b $\mathrm{Ar}=\beta-\mathrm{Mp}, \mathrm{R}^{1}=\mathrm{OMe}, \mathrm{R}^{2}=\mathrm{H}$

4c $A r=\beta-V e, R^{1}=H, \quad R^{2}=M e$

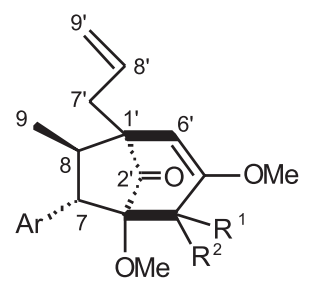

5a $\mathrm{Ar}=\mathrm{Pi}, \quad \mathrm{R}^{1}=\alpha-\mathrm{OH}, \mathrm{R}^{2}=\mathrm{H}$

$\mathbf{5 b} \mathrm{Ar}=\mathrm{Pi}, \quad \mathrm{R}^{1}=\beta-\mathrm{OH}, \mathrm{R}^{2}=\mathrm{H}$

$5 c \mathrm{Ar}=\mathrm{Pi}, \quad \mathrm{R}^{1} \mathrm{R}^{2}=\mathrm{O}$

$5 \mathrm{~d} A r=\mathrm{Mp}, \mathrm{R}^{1}=\alpha-\mathrm{OH}, \mathrm{R}^{2}=\mathrm{H}$

5e $\mathrm{Ar}=\mathrm{Mp}, \mathrm{R}^{1} \mathrm{R}^{2}=\mathrm{O}$

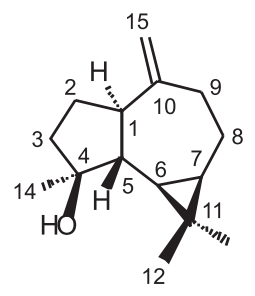

8 spathulenol

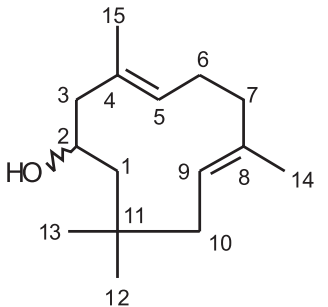

9

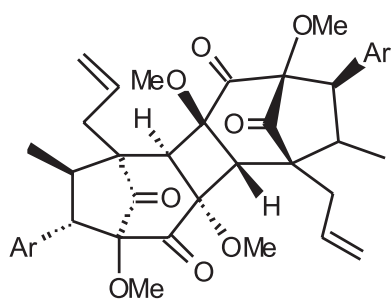

$7 \mathrm{a} \mathrm{Ar}=\mathrm{Pi}$

7b $\mathrm{Ar}=\mathrm{Mp}$

\footnotetext{
$\mathrm{Pi}=$ piperonyl (3,4-methylenedioxyphenyl)

Ve = veratryl $(3,4$-dimethoxyphenyl)

$\mathrm{Mp}=$ 5-methoxy-3,4-methylenedioxyphenyl

$\mathrm{Tp}=3,4,5$-trimethoxyphenyl
}

spathulenol $(\mathbf{8})^{4,14}$ were isolated as well. The embryogenic cultures were shown to contain a new benzofuran neolignan $\mathbf{4 a}$, six already known benzofuran $\mathbf{1 c},{ }^{6,12} \mathbf{1 d},{ }^{6} \mathbf{2 a},{ }^{6,10,11} \mathbf{2} \mathbf{b},{ }^{11,12}$ $\mathbf{2 e},{ }^{6}$ and a bicyclo[3.2.1] octane neolignan $\mathbf{6}^{15}$

\section{Results and Discussion}

The defatted fraction of hexane extracts from $O$. catharinensis leaves and from $O$. catharinensis somatic embryos were submitted to flash chromatography followed by prep. TLC and/or circular chromatography (Chromatotron $\AA)$. This procedure yielded nine new compounds (1e, 2f, 3b, 4a, 4b, 5c, 7a, 7b and 9), besides armenin-B (1a), ${ }^{6,10} \mathbf{1 c},{ }^{6,12}$ 5' $^{\prime}$-methoxyporosin (1d), ${ }^{6}$ ferrearin-C (2a) ${ }^{6,10,11}$ ferrearin-B (2b) $,{ }^{11,12} \mathbf{2 d},{ }^{4}$ ferrearin-E $(\mathbf{2 e}),{ }^{6}$ ferrearin-G $(\mathbf{2 g}),{ }^{12} \mathbf{5 e},{ }^{13} \mathbf{6}^{15}$ and spathulenol $(\mathbf{8}){ }^{4,14}$

The molecular formula of compound $\mathbf{1 e}$ was determined by HRESI as $\mathrm{C}_{23} \mathrm{H}_{30} \mathrm{O}_{7}$. Its ${ }^{1} \mathrm{H}$ NMR spectrum was quite similar to that of armenin-B (1a) $\left(\mathrm{C}_{21} \mathrm{H}_{24} \mathrm{O}_{6}\right)$, previously isolated from Licaria armeniaca ${ }^{10}$ and from 
Table 1. ${ }^{1} \mathrm{H}$ and ${ }^{13} \mathrm{C}$ NMR data of $\mathbf{1 e}, \mathbf{2 f}, \mathbf{3 b}, \mathbf{4 a}, \mathbf{4 b}$ and $\mathbf{6}\left[\delta, J(\mathrm{~Hz}), 200 \mathrm{MHz}\right.$ and $\left.50 \mathrm{MHz}, \mathrm{CDCl}_{3}\right]$

\begin{tabular}{|c|c|c|c|c|c|c|c|c|c|c|}
\hline \multirow[t]{2}{*}{ position } & \multicolumn{2}{|l|}{ 1e } & \multicolumn{2}{|l|}{$2 f$} & \multicolumn{2}{|l|}{$3 \mathbf{b}$} & \multirow{2}{*}{$\begin{array}{c}\mathbf{4 a} \\
{ }^{1} \mathrm{H}\end{array}$} & \multicolumn{2}{|l|}{$4 b$} & \multirow{2}{*}{$\frac{6}{{ }^{13} \mathrm{C}}$} \\
\hline & ${ }^{1} \mathrm{H}$ & ${ }^{13} \mathrm{C}$ & ${ }^{1} \mathrm{H}$ & ${ }^{13} \mathrm{C}$ & ${ }^{1} \mathrm{H}$ & ${ }^{13} \mathrm{C}$ & & ${ }^{1} \mathrm{H}$ & ${ }^{13} \mathrm{C}$ & \\
\hline 1 & & 131.8 & & 131.3 & & 131.3 & & & 132.5 & 129.4 \\
\hline 2 & 6.34 (brs) & 102.5 & $6.63-6.76(\mathrm{~m})$ & 110.8 & $7.38(\mathrm{~d}, 2.0)$ & 109.9 & $6.99(\mathrm{~d}, 1.5)$ & 6.45 (brs) & 106 & 113.9 \\
\hline 3 & & 153.4 & & 148.2 & & 148.7 & & & 148 & 148.2 \\
\hline 4 & & 136.1 & & 148.6 & & 149.9 & & & 134.2 & 147.3 \\
\hline 5 & & 153.3 & $6.63-6.76(\mathrm{~m})$ & 109.7 & $6.80(\mathrm{~d}, 8.4)$ & 110.8 & $6.64(\mathrm{~d}, 7.8)$ & & 143.1 & 110.6 \\
\hline 6 & 6.45 (brs) & 101.1 & $6.63-6.76(\mathrm{~m})$ & 118.5 & $7.52(\mathrm{dd}, 8.4 ; 2.0)$ & 122.8 & $6.73(\mathrm{dd}, 7.8 ; 1.5)$ & 6.42 (brs) & 106 & 122.8 \\
\hline 7 & $5.20(\mathrm{~d}, 1.9)$ & 92.5 & $5.36(\mathrm{~d}, 9.3)$ & 82.4 & & 202.3 & $4.43(\mathrm{~d}, 9.9)$ & $5.33(\mathrm{~d}, 9.7)$ & 82.3 & 57.1 \\
\hline 8 & $2.37-2.66(\mathrm{~m})$ & 44.3 & $2.75(\mathrm{~m})$ & 43.2 & $4.0(\mathrm{q}, 7.5)$ & 42.4 & $2.18-2.40(\mathrm{~m})$ & $2.72(\mathrm{~d}, 9.6)$ & 43.3 & 49.1 \\
\hline 9 & $1.15(\mathrm{~d}, 7.4)$ & 17.7 & $0.52(\mathrm{~d}, 7.4)$ & 11.4 & $1.10(\mathrm{~d}, 7.5)$ & 12.5 & $0.84(\mathrm{~d}, 6.8)$ & $0.57(\mathrm{~d}, 7.5)$ & 12.4 & 13.8 \\
\hline 1 , & & 47.3 & & 51.6 & & 50.4 & & & 49.9 & 50.8 \\
\hline 2 ' & & 31.6 & & 100.9 & & 196.5 & & & 102 & 77.4 \\
\hline 3 ' & & 77.3 & & 196.9 & & 152.8 & & & 152.5 & 89.9 \\
\hline $4^{\prime}$ & & 192.5 & $5.39(\mathrm{~s})$ & 102.4 & 5.72 (brs) & 132.9 & 4.78 (brs) & 4.92 (brs) & 97.5 & 193.7 \\
\hline 5 & $3.93(\mathrm{dd}, 12.1 ; 5.0)$ & 132.3 & & 169.3 & $4.3(\mathrm{~m})$ & 72.8 & $4.03-4.12(\mathrm{~m})$ & $4.08-4.13(\mathrm{~m})$ & 74.3 & 151.8 \\
\hline \multirow[t]{2}{*}{6} & $1.82(\mathrm{dd}, 12.0 ; 12.0)$ & 169.7 & $2.45(\mathrm{~d}, 16.5)$ & 41.7 & $2.80(\mathrm{dd}, 12.9 ; 9.9)$ & 34.6 & $2.00(\mathrm{dd}, 12.6 ; 5.0)$ & $1.90(\mathrm{dd}, 12.3 ; 4.8)$ & 31.7 & 119.3 \\
\hline & $2.09-2.25(\mathrm{~m})$ & & & & $2.20(\mathrm{dd}, 12.9 ; 5.3)$ & & $1.40(\mathrm{dd}, 12.6 ; 10.0)$ & $1.46(\mathrm{dd}, 10.0 ; 10.0)$ & & \\
\hline \multirow[t]{2}{*}{7} & $2.09-2.25(\mathrm{~m})$ & 41.3 & $2.31(\mathrm{dd}, 7.3 ; 5.5)$ & 40.1 & $2.50(\mathrm{dd}, 14.4 ; 6.4)$ & 39 & $2.18-2.40(\mathrm{~m})$ & $2.43(\mathrm{dd}, 14.2 ; 7.1)$ & 38.4 & 36.9 \\
\hline & & & & & $2.30(\mathrm{dd}, 14.4 ; 8.8)$ & & & $2.23(\mathrm{dd}, 14.2 ; 7.9)$ & & \\
\hline 8 & $5.54-5.65(\mathrm{~m})$ & 132.3 & $5.64-5.73(\mathrm{~m})$ & 133.4 & $5.58-5.68(\mathrm{~m})$ & 132.9 & $5.86-5.95(\mathrm{~m})$ & $5.90-5.95(\mathrm{~m})$ & 135 & 133.8 \\
\hline \multirow[t]{2}{*}{ 9' } & $5.03(\mathrm{dd}, 14.6 ; 1.4)$ & 120 & $5.00(\mathrm{dd}, 15.3 ; 1.9)$ & 118.7 & $5.05(\mathrm{dd}, 12.0 ; 2.0)$ & 118.6 & $5.03(\mathrm{dd}, 14.8 ; 1.8$ & $5.12(\mathrm{dd}, 14.4 ; 1.8)$ & 117.5 & 118.8 \\
\hline & $4.72(\mathrm{dd}, 11.2 ; 1.4)$ & & $4.93(\mathrm{dd}, 10.0 ; 1.9)$ & & & & $5.03(\mathrm{dd}, 14.8 ; 1.8$ & $5.08(\mathrm{dd}, 7.7 ; 1.8)$ & & \\
\hline $\mathrm{CH}_{2} \mathrm{O}_{2}$ & & & & & & & $5.86(\mathrm{~s})$ & $5.92(\mathrm{~s})$ & 101.3 & \\
\hline $\mathrm{MeO}-3$ & $3.80(\mathrm{~s})$ & 60.9 & $3.79(\mathrm{~s})$ & 55.8 & $3.82(\mathrm{~s})$ & 55.9 & & & & 55.8 \\
\hline $\mathrm{MeO}-4$ & $3.81(\mathrm{~s})$ & 60.5 & $3.78(\mathrm{~s})$ & 55.9 & $3.86(\mathrm{~s})$ & 55.8 & & & & 55.7 \\
\hline $\mathrm{MeO}-5$ & $3.82(\mathrm{~s})$ & 60.9 & & & & & & $3.85(\mathrm{~s})$ & 56.7 & \\
\hline $\mathrm{MeO}-3$ & $3.73(\mathrm{~s})$ & 59.3 & & & $3.58(\mathrm{~s})$ & 55.7 & $3.61(\mathrm{~s})$ & $3.67(\mathrm{~s})$ & 55.8 & 54.7 \\
\hline $\mathrm{MeO}-4$ & & & & & $3.34(\mathrm{~s})$ & 55 & & & & \\
\hline $\mathrm{MeO}-5$ ' & $3.56(\mathrm{~s})$ & 55.9 & $3.75(\mathrm{~s})$ & 55.6 & & & $3.34(\mathrm{~s})$ & $3.37(\mathrm{~s})$ & 55.1 & 55.5 \\
\hline $\mathrm{OH}-2$ ' & & & $3.66(\mathrm{~s})$ & & & & & & & \\
\hline COMe-4' & & & $3.66(\mathrm{~s})$ & & & & & & & $21.0 / 169.0$ \\
\hline
\end{tabular}

O. catharinensis. ${ }^{6}$ The only difference was assigned to the oxygenation pattern of the aromatic ring, which was determined as 3,4,5-trimethoxyphenyl in 1e instead of 3,4-methylenedioxyphenyl in 1a.

The ${ }^{1} \mathrm{H}$ NMR spectra of $\mathbf{2 f}\left(\mathrm{C}_{21} \mathrm{H}_{26} \mathrm{O}_{6}\right)$ and of ferrearin-E (2e) $\left(\mathrm{C}_{20} \mathrm{H}_{24} \mathrm{O}_{5}\right)$ isolated from $O$. catharinensis ${ }^{6}$ were similar. The difference between both compounds consisted of an additional methoxyl group at $\beta$-position (C-5', $\delta$ 169.3) of $\mathbf{2 f}$.

The molecular formula of compound $\mathbf{3 b}$ was determined by HRESI as $\mathrm{C}_{22} \mathrm{H}_{28} \mathrm{O}_{6}$. All the ${ }^{1} \mathrm{H}$ NMR data was similar to that of a neolignan 3a $\left(\mathrm{C}_{21} \mathrm{H}_{26} \mathrm{O}_{5}\right)$ previously isolated from $O$. porosa.${ }^{16}$ Nevertheless, the ${ }^{1} \mathrm{H}$ and ${ }^{13} \mathrm{C}$ NMR spectra indicated that $\mathbf{3 b}$ contained a methoxyl group at
C-3' $\left(\delta_{\mathrm{H}} 3.58, \delta_{\mathrm{C}} 55.7\right)$ which was evidenced by the signal of C-3' at $\delta_{\mathrm{C}} 152.8$ instead of $\delta_{\mathrm{C}} 132.7 \mathrm{in}$ 3a. Its absolute configuration was determined as $8 S, 1^{\prime} R, 5^{\prime} R$ based on the signal of optical rotation $\left([\alpha]_{D}^{21}=-17^{\circ}(\mathrm{MeOH}\right.$, $c=0.92 \mathrm{~g} / 100 \mathrm{~mL}$ ) and comparison with reported data for (-)-megaphone in which X-ray crystallographic studies was carried out. ${ }^{16}$

The compounds $\mathbf{4 a}$ and $\mathbf{4 b}$ were characterized as hexahydrobenzofuran neolignans by analysis of their ${ }^{1} \mathrm{H}$ and ${ }^{13} \mathrm{C}$ NMR spectra and comparison with $\mathbf{4 c}$ which was previously isolated from $O$. poros $a .{ }^{9}$ The aromatic rings were determined as 3,4-methylenedioxyphenyl and 5-methoxy3,4-methylenedioxyphenyl for $\mathbf{4 a}$ and $\mathbf{4 b}$, respectively. The relative stereochemistry between methyl and aryl groups 
was deduced from the anisotropic shielding effect caused by aromatic ring on cis methyl hydrogens as observed in the ${ }^{1} \mathrm{H}$ NMR spectra. The methyl (H-9) signals of $\mathbf{4 a}$ (trans) and 4b (cis) were observed at $\delta 0.84(\mathrm{~d}, 6.8 \mathrm{~Hz})$ and $0.57(\mathrm{~d}, 7.5$ $\mathrm{Hz}$ ), respectively. The placement of hydroxyl group at C-2' and methoxyl group at C-3' were possible by comparison of ${ }^{13} \mathrm{C}$ NMR data and those described for $\mathbf{4 c} .{ }^{9}$

The molecular formula of compound $\mathbf{5} \mathbf{c}$ was determined by HRESI as $\mathrm{C}_{21} \mathrm{H}_{22} \mathrm{O}_{6}$. Its ${ }^{1} \mathrm{H}$ NMR were similar to that observed for previously reported $\mathbf{5 e}\left(\mathrm{C}_{22} \mathrm{H}_{24} \mathrm{O}_{7}\right)$ isolated from Aniba simulans, ${ }^{13}$ but with a 3,4-methylenedioxy for $\mathbf{5 c}$ instead of 5-methoxy-3,4-methylenedioxy observed for 5 e.

Compounds $\mathbf{7 a}$ and $\mathbf{7 b}$ had their structures determined by analysis of their IR, MS and NMR spectra. The IR spectra of 7a/7b exhibited absorption bands at 1766 and $1714 \mathrm{~cm}^{-1}$, assignable to two carbonyl groups for each compound. Their ${ }^{1} \mathrm{H}$ and ${ }^{13} \mathrm{C}$ NMR spectra resembled those of $\mathbf{5} \mathbf{c} / \mathbf{5 e}$, respectively. Nevertheless, the ${ }^{13} \mathrm{C}$ NMR signals associated to the olefinic double bond at $\mathrm{C}^{\prime}-\mathrm{C6}^{\prime}$ were replaced by signals of quaternary carbinolic and methine signals at $\delta 88.4 / 52.6$ (7a) and $\delta 88.4 / 52.7$ (7b), respectively. The EIMS of $7 \mathbf{a} / 7 \mathbf{b}$ showed molecular ion at $m / z, 370$ and 400 , but the chemical ionization mass spectrometry (CIMS) provided a molecular ion peak $(\mathrm{M}+\mathrm{H})^{+}$at $\mathrm{m} / z .741$ and $m / z$ 801, respectively. These molecular ions combined with ${ }^{1} \mathrm{H}$ and ${ }^{13} \mathrm{C}$ NMR (PND and DEPT $135^{\circ}$ ), suggested a molecular formula $\mathrm{C}_{42} \mathrm{H}_{44} \mathrm{O}_{12}$ and $\mathrm{C}_{44} \mathrm{H}_{48} \mathrm{O}_{14}$ compatible with dimeric structures. Thus, the fragmentary ion peaks found at $\mathrm{m} / \mathrm{z} 371$ of $\mathbf{7 a}$ and 401 of $\mathbf{7 b}$ were assigned to the cleavage into monomers indicating that $\mathbf{7 a}$ and $\mathbf{7 b}$ were symmetric dimers of $\mathbf{5 a}$ and $\mathbf{5 e}$. Based on similarities to 5a and 5e all ${ }^{1} \mathrm{H}$ and ${ }^{13} \mathrm{C}$ NMR data were assigned with the aid of ${ }^{1} \mathrm{H}-{ }^{1} \mathrm{H}$ COSY and HETCOR spectra (Table 2). In order to determine the relative stereochemistry at the cyclobutane ring, NOESY spectra showed cross-peaks between H-8 and H-7', H-7' and H-6', H-6' and OMe-5'. Thus, among the cyclobutane syn-adducts at C-5' and C-6' four dimers would be expected (Figure 1). Dimers III and IV having cis configuration at the cyclobutane would involve a considerable steric hindrance between the bulky groups of bicyclooctane neolignan, which would prevent such arrangement. For these reason, dimers I and II having trans configuration were considered as the mostly probable structures.

The molecular formula of $9\left(\mathrm{C}_{15} \mathrm{H}_{26} \mathrm{O}\right)$ was deduced from MS and ${ }^{13} \mathrm{C} \mathrm{NMR}$ (PND and DEPT 135 $)$ spectral data. The spectral characteristics of compound 9 were closely related to those of $\alpha$-humulane ${ }^{17}$ except for the existence of a hydroxyl group (IR $v_{\max } / \mathrm{cm}^{-1}: 3425$ ) and two double bonds instead of three. All the ${ }^{1} \mathrm{H}$ and ${ }^{13} \mathrm{C}$ NMR signals were assigned by ${ }^{1} \mathrm{H}-{ }^{1} \mathrm{H}$
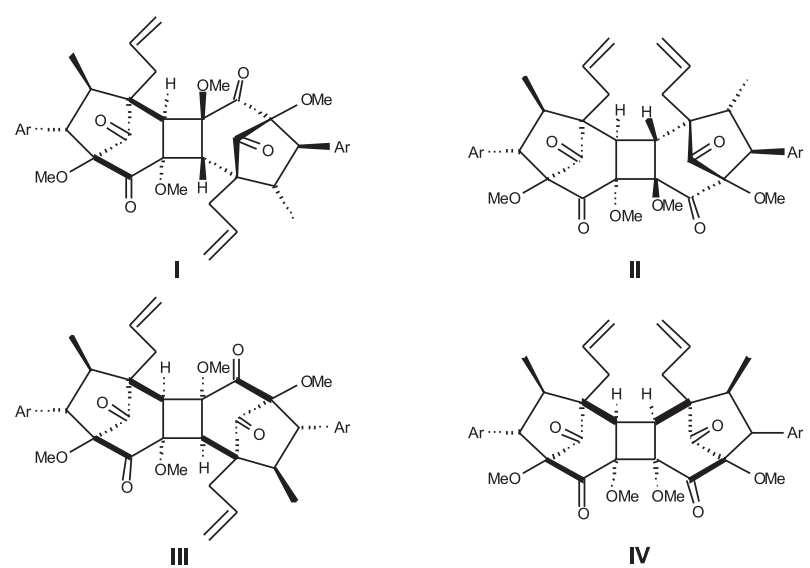

II

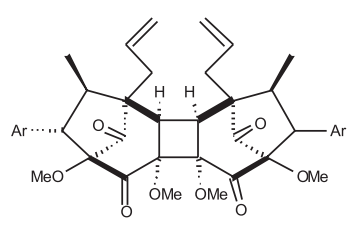

IV

Figure 1. Possible structure of dimers for $\mathbf{7 a} / \mathbf{7 b}$.

COSY and HETCOR techniques and were compatible with the structure depicted for humula-4,8-dien-2-ol (9).

The leaves of $O$. catharinensis collected in São Paulo and in Santa Catarina were showed to contain both benzofuran and bicyclo[3.2.1] octane type neolignans. The benzofuran neolignan $\mathbf{1 d}$ was the major compound in the leaves collected in São Paulo while bicyclo[3.2.1]octane neolignans $\mathbf{5 e / 5}$ c were predominant in Santa Catarina specimen. The embryogenic cultures also produced both types of neolignans, but 1d was the major compound.

\section{Experimental}

\section{General}

Prep. TLC was carried out on silica gel PF-254 (Merck) and CC on silica gel 60H (0.005-0.045 mm) (Merck). Optical rotations were measured on Jasco Mod. DIP-370. ${ }^{1} \mathrm{H}$ and ${ }^{13} \mathrm{C}$ NMR spectra were registered on a Bruker AC-200 spectrometers using $\mathrm{CDCl}_{3}$ as solvent and TMS as internal standard. EIMS $(70 \mathrm{eV})$ and CIMS (methane) were obtained on a HP 5988-A spectrometer. HRESIMS (positive mode) were recorded on a Bruker Daltonics microTOF. Elemental analysis were performed on a Perkin-Elmer CHN Elemental Analyser 2400. HPLC were performed on Gilson 321 using a reverse phase column (Supelco, C-18, $4.6 \times 250 \mathrm{~mm}, 5 \mu \mathrm{m}$ ).

\section{Plant material}

The leaves of the $O$. catharinensis were collected in Brusque, Santa Catarina State, Brazil in June 1993. The species was collected and identified by Prof. Ademir Reis (Departamento de Botânica, UFSC). The voucher specimen (FLOR-17560) was deposited in the FLOR Herbarium (UFSC). 
Table 2. ${ }^{1} \mathrm{H}$ and ${ }^{13} \mathrm{C}$ NMR of compounds $\mathbf{5 c}, \mathbf{5 e}, 7 \mathbf{a}$ and $\mathbf{7 b}\left[\delta, J(\mathrm{~Hz}), 200 \mathrm{MHz}\right.$ and $\left.50 \mathrm{MHz}, \mathrm{CDCl}_{3}\right]$

\begin{tabular}{|c|c|c|c|c|c|c|c|c|}
\hline \multirow[t]{2}{*}{ position } & \multicolumn{2}{|l|}{$5 \mathrm{c}$} & \multicolumn{2}{|l|}{$5 e$} & \multicolumn{2}{|l|}{$7 \mathbf{a}$} & \multicolumn{2}{|l|}{$7 b$} \\
\hline & ${ }^{1} \mathrm{H}$ & ${ }^{13} \mathrm{C}$ & ${ }^{1} \mathrm{H}$ & ${ }^{13} \mathrm{C}$ & ${ }^{1} \mathrm{H}$ & ${ }^{13} \mathrm{C}$ & ${ }^{1} \mathrm{H}$ & ${ }^{13} \mathrm{C}$ \\
\hline 1 & & 130.8 & & 131.5 & & 131.8 & & 132.5 \\
\hline 2 & 6.51 (brs) & 109.4 & $6.14(\mathrm{~d}, 1.4)$ & 109.4 & $6.50(\mathrm{~s})$ & 107.9 & $6.13(\mathrm{~s})$ & 108.9 \\
\hline 3 & & 146.9 & & 148.7 & & 147.1 & & 142.9 \\
\hline 4 & & 147.6 & & 134.4 & & 147.4 & & 148.6 \\
\hline 5 & $6.69(\mathrm{~d}, 7.6)$ & 107.9 & & 142.9 & $6.70(\mathrm{~d}, 8.4)$ & 108.9 & & 108.9 \\
\hline 6 & $6.49(\mathrm{~d}, 7.6)$ & 122.9 & $6.17(\mathrm{~d}, 1.4)$ & 103.1 & $6.49(\mathrm{dd}, 8.4 ; 1.8)$ & 122.2 & $6.13(\mathrm{~s})$ & 122.2 \\
\hline 7 & $2.54-2.59(\mathrm{~m})$ & 53.9 & $2.46-2.51(\mathrm{~m})$ & 54.1 & $2.89(\mathrm{~d}, 4.5)$ & 62.2 & $2.81(\mathrm{~d}, 4.5)$ & 62.4 \\
\hline 8 & $2.19-2.24(\mathrm{~m})$ & 47.1 & $2.15-2.22(\mathrm{~m})$ & 47.0 & $2.53(\mathrm{dd}, 6.7 ; 4.5)$ & 37.2 & $2.53(\mathrm{dd}, 7.0 ; 4.5)$ & 37.2 \\
\hline 9 & $0.96(\mathrm{~d}, 6.6)$ & 14.3 & $0.92(\mathrm{~d}, 6.7)$ & 14.4 & $1.18(\mathrm{~d}, 6.7)$ & 18.9 & $1.12(\mathrm{~d}, 7.0)$ & 18.9 \\
\hline 1 ' & & 54.2 & & 54.2 & & 56.6 & & 56.5 \\
\hline 2 & & 204.9 & & 204.9 & & $201.6^{*}$ & & $201.5^{*}$ \\
\hline $3^{\prime}$ & & 93.7 & & 93.6 & & $85.2 * *$ & & $85.2 * *$ \\
\hline $4^{\prime}$ & & 191.7 & & 191.7 & & $199.2 *$ & & $198.1 *$ \\
\hline 5 , & & 152.7 & & 152.8 & & $88.4^{* *}$ & & $88.4 * *$ \\
\hline $6^{\prime}$ & $5.81(\mathrm{~s})$ & 117.7 & $5.76(\mathrm{~s})$ & 117.8 & $2.96(\mathrm{~s})$ & 52.6 & $2.91(\mathrm{~s})$ & 52.7 \\
\hline $7^{\prime}$ & $2.52-2.58(\mathrm{~m})$ & 35.8 & $2.46-2.51(\mathrm{~m})$ & 35.7 & $2.23(\mathrm{dd}, 15.2 ; 5.7)$ & 34.4 & $2.20(\mathrm{dt}, 5.7 ; 2.9 ; 1.4)$ & 34.3 \\
\hline & & & & & $2.65(\mathrm{dd}, 15.2 ; 8.4)$ & & $2.45(\mathrm{dd}, 6.7 ; 4.6)$ & \\
\hline 8, & $5.87-5.96(\mathrm{~m})$ & 132.9 & $5.82-5.91 \mathrm{~m}$ & 132.9 & $5.80-5.92(\mathrm{~m})$ & 132.6 & $5.80-5.87(\mathrm{~m})$ & 132.6 \\
\hline $9^{\prime}$ & $5.19(\mathrm{~d}, 17.0)$ & 119.3 & $5.13(\mathrm{~d}, 16.6)$ & 119.3 & $5.20(\mathrm{~d}, 15.7)$ & 119.6 & $5.20(\mathrm{~d}, 15.7)$ & 119.6 \\
\hline & $5.17(\mathrm{~d}, 10.3)$ & & $5.11(\mathrm{~d}, 10.6)$ & & $5.23(\mathrm{~d}, 11.2)$ & & $5.25(\mathrm{~d}, 11.6)$ & \\
\hline $\mathrm{CH}_{2} \mathrm{O}_{2}$ & $5.91(\mathrm{~d}, 2.3)$ & 101.1 & $5.88(\mathrm{~d}, 1.3)$ & 101.3 & $5.91(\mathrm{~s})$ & 101.1 & $5.86(\mathrm{~d}, 1.5)$ & 101.4 \\
\hline $\mathrm{MeO}-5$ & - & - & $3.78(\mathrm{~s})$ & 55.8 & - & - & $3.78(\mathrm{~s})$ & 56.1 \\
\hline MeO-3' & $3.52(\mathrm{~s})$ & 56.3 & $3.47(\mathrm{~s})$ & 56.3 & $3.35(\mathrm{~s})$ & 55.1 & $3.31(\mathrm{~s})$ & 55.2 \\
\hline MeO-5' & $3.73(\mathrm{~s})$ & 53.9 & $3.67(\mathrm{~s})$ & 56.5 & $3.61(\mathrm{~s})$ & 58.4 & $3.55(\mathrm{~s})$ & 58.4 \\
\hline
\end{tabular}

***: Assignments may be reversed.

Table 3. ${ }^{1} \mathrm{H}$ and ${ }^{13} \mathrm{C}$ NMR data of compound $9[\delta, J(\mathrm{~Hz}), 200 \mathrm{MHz}$ and $50 \mathrm{MHz}, \mathrm{CDCl}_{3}$ ]

\begin{tabular}{lcc}
\hline position & \multicolumn{2}{c}{9} \\
\cline { 2 - 3 } & ${ }^{1} \mathrm{H}$ & ${ }^{13} \mathrm{C}$ \\
\hline 1 & $4.83-4.85$ & 124.7 \\
2 & $1.75-2.0(\mathrm{~m})$ & 25.2 \\
3 & $1.75-2.2(\mathrm{~m})$ & 39.2 \\
4 & & 133.0 \\
5 & $4.83-4.85(\mathrm{~m})$ & 126.5 \\
6 & $0.90-1.1(\mathrm{~m})$ & 47.0 \\
& $1.75-2.0(\mathrm{~m})$ & \\
7 & $1.9-2.2(\mathrm{~m})$ & 49.7 \\
8 & $3.14(\mathrm{ddd} ; 2.9 ; 2.7 ; 2.4)$ & 70.0 \\
9 & $1.75-2.2(\mathrm{~m})$ & 39.3 \\
10 & & 132.2 \\
11 & & 33.6 \\
12 & $0.95(\mathrm{~s})$ & 31.2 \\
13 & $1.05(\mathrm{~s})$ & 27.7 \\
14 & $1.44(\mathrm{~s})$ & 15.9 \\
15 & $1.59(\mathrm{~s})$ & 18.3 \\
\hline
\end{tabular}

\section{Initiation and multiplication of embryogenic cultures}

Embryogenic cultures were initiated from mature zygotic embryos of $O$. catharinensis according to described methodology. ${ }^{1,2}$

The somatic embryos produced at early cotyledonary stage (2-3 mm length) were inoculated in Woody Plant Medium (WPM, Sigma Co., USA) supplemented with $22.7 \mathrm{~g} \mathrm{~L}^{-1}$ sorbitol, $2 \mathrm{~g} \mathrm{~L}^{-1}$ Phytagel, $20 \mathrm{~g} \mathrm{~L}^{-1}$ sucrose and $400 \mathrm{mg} \mathrm{L}^{-1}$ glutamine ( $\mathrm{pH} 5.8$ ) and maintained at $25^{\circ} \mathrm{C}$ and with photon flux of $22 \mu \mathrm{mol} \mathrm{m} \mathrm{m}^{-2} \mathrm{~s}^{-1}$ provided by fluorescent tubes under $16 \mathrm{~h}$ photoperiod. ${ }^{2,18}$ After four weeks cultivation the somatic embryos at mature stage $(\geq 5 \mathrm{~mm})$ were transferred to Petri dishes $(6 \mathrm{~cm})$, spread over two filter papers, and maintained at $25^{\circ} \mathrm{C}$ for 4 days for desiccation.

\section{Extraction and isolation of the constituents from leaves}

Dried and powdered leaves (395.0 g) were exhaustively extracted with hexane at room temp. Evaporation of the 
hexane under a reduced pressure gave a residue, which was partitioned between hexane and $\mathrm{MeOH}-\mathrm{H}_{2} \mathrm{O}(9: 1)$. The hydroalcoholic phase was concentrated under reduced pressure yielding $2.4 \mathrm{~g}$. This residue was submitted to flash chromatography column (silica gel, $150 \mathrm{~g}$ ) and eluted with hexane-EtOAc mixtures at increasing polarities (7:3 to $0: 1$ ), yielding 120 fractions (30 mL each). Frs. 29-32 $\left(33.0 \mathrm{mg}\right.$ ) submitted to prep. TLC ( silica gel, hexane- $\mathrm{Et}_{2} \mathrm{O}$, 4:1) gave spathulenol $8(7.0 \mathrm{mg})$ and humula-4,8-dien-2-ol 9 (17.0 mg). Frs. 33-41 (76.0 mg) was also fractionated by prep. TLC (silica gel, cyclohexane- $\mathrm{Me}_{2} \mathrm{CO}, 98: 2$ ) followed by prep. TLC (silica gel, hexane- $\mathrm{CHCl}_{3}$-iso-PrOH, 94.5:5.0:0.5) and afforded $\mathbf{2 d}(5.5 \mathrm{mg})$ and $\mathbf{2 a}(4.9 \mathrm{mg})$. Frs. $44-59(60.0 \mathrm{mg})$ submitted to prep. TLC (silica gel, cyclohexane- $\left.\mathrm{Me}_{2} \mathrm{CO}, 97: 3\right)$ followed by prep. TLC (silica gel, $\mathrm{CHCl}_{3}$-EtOAc-iso-PrOH, 94.5:5.0:0.5) gave $\mathbf{2 g}(3.0 \mathrm{mg})$ and $\mathbf{6}(6.0 \mathrm{mg})$. Fr. $60(24.0 \mathrm{mg})$ fractionated by prep. TLC (silica gel, cyclohexane-Me ${ }_{2} \mathrm{CO}, 98: 2$ ) yielded 5c (6.0 mg). Frs. 61-65 (440.0 mg) submitted to flash chromatography column (silica gel, $\mathrm{CHCl}_{3}$ EtOAc-iso-PrOH, 89:10:1), affording three sub-fractions $\left(\mathrm{S}_{1}-\mathrm{S}_{3}\right)$. Prep. TLC of sub-fraction $\mathrm{S}_{1}(70.0 \mathrm{mg}$ ) (silica gel, $\mathrm{CHCl}_{3}$-EtOAc-iso-PrOH, 94.5:5.0:0.5) afforded 7a $(17.0 \mathrm{mg})$ and prep. TLC of sub-fraction $\mathrm{S}_{3}(80.0 \mathrm{mg})$ (silica gel, $\mathrm{CHCl}_{3}$-EtOAc-iso-PrOH, 94.5:5.0:0.5) yielded 5c $(30.0 \mathrm{mg})$. Frs. 66-71 (272.0 mg) fractionated by prep. TLC [silica gel, cyclohexane- $\mathrm{Me}_{2} \mathrm{CO}$ (97:3)] furnished $\mathbf{2 e}$ (6.0 mg). Frs. 77-79 (150.0 mg) submitted to chromatotron (silica gel, hexane-EtOAc-iso-PrOH, 69:30:1), to give two sub-fractions $\left(\mathrm{S}_{4}\right.$ and $\left.\mathrm{S}_{5}\right)$. Prep. TLC of sub-fraction $\mathrm{S}_{4}$ $(14.0 \mathrm{mg})$ (silica gel, cyclohexane- $\left.\mathrm{Me}_{2} \mathrm{CO}, 95: 5\right)$ gave 2a $(5.0 \mathrm{mg})$ and $7 \mathbf{b}(4.0 \mathrm{mg})$ and prep. TLC of sub-fraction $\mathrm{S}_{5}\left(98.0 \mathrm{mg}\right.$ ) (silica gel, cyclohexane- $\left.\mathrm{Me}_{2} \mathrm{CO}, 95: 5\right)$ gave 5e $(70.0 \mathrm{mg})$. Frs. $80-81(98.0 \mathrm{mg})$ submitted to prep. TLC (silica gel, $\left.\mathrm{CH}_{2} \mathrm{Cl}_{2}-\mathrm{Me}_{2} \mathrm{CO}, 95: 5\right)$ yielded $4 \mathbf{b}$ (12.0 mg). Fr. $82-86(500.0 \mathrm{mg})$ submitted to chromatotron (silica gel, hexane-EtOAc-iso-PrOH, 89:10:1) afforded 2e (7.2 mg) and two sub-fractions $\mathrm{S}_{6}$ and $\mathrm{S}_{7}$. Sub-fraction $\mathrm{S}_{6}(400.0 \mathrm{mg})$ was further fractionated by flash chromatography column (silica gel, $\left.\mathrm{CH}_{2} \mathrm{Cl}_{2}-\mathrm{Me}_{2} \mathrm{CO}, 98: 2\right)$ yielding $2 \mathbf{2}(12.0 \mathrm{mg}$ ) and $\mathbf{3 b}(9.0 \mathrm{mg})$. Sub-fraction $\mathrm{S}_{7}(43.0 \mathrm{mg})$ was submitted to chromatotron (silica gel, $\mathrm{CH}_{2} \mathrm{Cl}_{2}-\mathrm{Me}_{2} \mathrm{CO}, 95: 5$ ) to give 1a $(24.0 \mathrm{mg})$. Frs. $105-110(87.0 \mathrm{mg})$ was submitted to chromatotron (silica gel, $\left.\mathrm{CH}_{2} \mathrm{Cl}_{2}-\mathrm{Me}_{2} \mathrm{CO}, 96: 4\right)$ yielding 1d and 1 e $(5.5 \mathrm{mg})$ as a mixture.

Extraction and isolation of the constituents from somatic embryos

The desiccated somatic embryos $(400.0 \mathrm{~g}$ ) were frozen in $\mathrm{Me}_{2} \mathrm{CO}$ with dry ice, ground and extracted with $500 \mathrm{~mL}$
$\mathrm{MeOH}-\mathrm{H}_{2} \mathrm{O}$ (4:1). The hydroalcoholic extracts was concentrated and successively partitioned with hexane and $\mathrm{CHCl}_{3}\left(3 \times 200 \mathrm{~mL}\right.$, each). The $\mathrm{CHCl}_{3}$ residue $(360.0 \mathrm{mg})$ was submitted to flash chromatography column (silica gel, $150 \mathrm{~g}$ ) and eluted with $\mathrm{CH}_{2} \mathrm{Cl}_{2}-\mathrm{Me}_{2} \mathrm{O}$ at increasing polarity (1:1 to $0: 1)$, affording 160 fractions (20 $\mathrm{mL}$ each). Fr. 1 $\left(18.0 \mathrm{mg}\right.$ ) submitted to prep. TLC (cyclohexane- $\mathrm{Me}_{2} \mathrm{CO}$, 95:5) yielded $2 \mathbf{a}(5.0 \mathrm{mg})$ and $\mathbf{6}(5.0 \mathrm{mg})$. Frs. 2-12 (7.0 $\mathrm{mg}$ ) submitted to prep. TLC (cyclohexane- $\mathrm{Me}_{2} \mathrm{CO}, 95: 5$ ) gave 2a (1.0 mg) and 2e (2.0 mg). Frs. 13-21 (20.5 mg) submitted to prep. TLC (cyclohexane- $\left.\mathrm{Me}_{2} \mathrm{CO}, 95: 5\right)$ gave 2b (3.0 mg), 2a (4.0 mg) and 1c (3.0 mg). Frs. 22-54 (43.0 $\mathrm{mg})$ purified by prep. TLC $\left(\mathrm{CH}_{2} \mathrm{Cl}_{2}-\mathrm{Me}_{2} \mathrm{O}, 9: 1\right)$ yielded 4a $(3.0 \mathrm{mg})$ and $\mathbf{6}(4.0 \mathrm{mg})$. Frs. 145-158 (29.0 mg) fractionated by prep. TLC $\left(\mathrm{CHCl}_{3}-\mathrm{MeOH}, 9: 1\right)$ yielded 1d $(7.0 \mathrm{mg})$.

(7S, 8S, 1'R, 3'R)-3,4,5,3', 5'-Pentamethoxy-4'-oxo- $\Delta^{1,3,5,5,8^{\prime}}$ 8.1',7.O.6'-neolignan (1e)

Viscous oil; IR (film) $v_{\max } / \mathrm{cm}^{-1}: 3469,2925,1707,1695$, $1500,1446,1371,1218,1087,1033,925,816,772 ;{ }^{1} \mathrm{H}$ and ${ }^{13} \mathrm{C}$ NMR, see Table 1; HRESIMS $m / z: 419.2090[\mathrm{M}+\mathrm{H}]^{+}$ (calcd for $\left.\mathrm{C}_{23} \mathrm{H}_{31} \mathrm{O}_{7}, 419.2071\right)$; EIMS $(70 \mathrm{eV}) \mathrm{m} / \mathrm{z}$ (rel. int.): 418( $\left.\mathrm{M}^{+}, 34\right), 388(19), 377(100), 349(87), 317(29)$, 285(13), 208(44), 181(22), 91(13).

$\operatorname{rel}(7 R, 8 S, 1$ ' $R, 2$ ''S)-2'-Hydroxy-3,4,5'-trimethoxy-3'-oxo$\Delta^{1,3,3,5,4^{\prime}, 8^{\prime}-8.1}$ ', 7.O.2'-neolignan (2f)

Viscous oil; $[\alpha]_{D}^{21}=-130^{\circ}(\mathrm{MeOH}, c=0.20 \mathrm{~g} / 100 \mathrm{~mL})$; IR (film) $v_{\max } / \mathrm{cm}^{-1}: 3435,2936,2849,1739,1664,1588,1511$, 1457, 1251, 1142, 1012, 762; ${ }^{1} \mathrm{H}$ and ${ }^{13} \mathrm{C}$ NMR, see Table 1; EIMS (70 eV) m/z (rel. int.): 374(M+1 7), 194(17), 167(100), 166(66), 165(76), 139(56), 95(17), 77(21), 69(18).

rel ( $\left.8 S, I^{\prime} R, 5^{\prime} R\right)-3,4,3^{\prime}, 5^{\prime}$-Tetramethoxy-7,2'-dioxo$\Delta^{1,3,5,3^{\prime}, 8^{\prime}-8.1^{\prime} \text { '-neolignan }(3 \boldsymbol{b})}$

Viscous oil; $[\alpha]_{D}^{21}=-17^{\circ}(\mathrm{MeOH}, c=0.92 \mathrm{~g} / 100 \mathrm{~mL})$; IR (film) $v_{\max } / \mathrm{cm}^{-1}: 2957,2914,2860,2348,1739,1620$, $1511,1457,1371,1263,1229,1144,1023,766 ;{ }^{1} \mathrm{H}$ and ${ }^{13} \mathrm{C}$ NMR, see Table 1; HRESIMS m/z: $389.1983[\mathrm{M}+\mathrm{H}]^{+}$ (calcd for $\left.\mathrm{C}_{22} \mathrm{H}_{29} \mathrm{O}_{6}, 389.1966\right)$; EIMS $(70 \mathrm{eV}) \mathrm{m} / \mathrm{z}$ (rel. int.): 388( $\left(\mathrm{M}^{+}, 4\right), 352(30), 339(23), 324(10), 316(3)$, 165(100), 137(6).

rel(7S, 8S, 1'R, 2'S)-2'-Hydroxy-3,4-methylenedioxy-3',5'dimethoxy- $\Delta^{1,3,5,3,3^{\prime}, 8^{\prime}-8.1}$ ', 7.O.2'-neolignan (4a)

Viscous oil; ${ }^{1} \mathrm{H}$ NMR see Table 1.

$\operatorname{rel}(7 R, 8 S, 1$ ' $R, 2$ 'S)-2'-Hydroxy-3,4-methylenedioxy5,3',5'-trimethoxy- $\Delta^{1,3,5,3, \text {, }^{\prime}-8.1}$ ', 7.O.2'-neolignan (4b)

Viscous oil; ${ }^{1} \mathrm{H}$ and ${ }^{13} \mathrm{C}$ NMR, see Table 1. 
$\operatorname{rel}(7 S, 8 R, 1$ 'R, 3'R)-4'-Hydroxy-3,4-methylenedioxy-3',5'dimethoxy-2',4'-dioxo- $\Delta^{1,3,5,5,5^{\prime}}$ '-8.1', 7.3'-neolignan (5c)

Viscous oil; $[\alpha]_{D}^{21}=-18^{\circ}(\mathrm{MeOH}, c=3.75 \mathrm{~g} / 100 \mathrm{~mL})$; IR (film) $v_{\max } / \mathrm{cm}^{-1}: 1765,1698,1504,1491,1247,1094,1039$. ${ }^{1} \mathrm{H}$ and ${ }^{13} \mathrm{C}$ NMR, see Table 2; HRESIMS $m / z$ : 371.1496 $[\mathrm{M}+\mathrm{H}]^{+}$(calcd for $\left.\mathrm{C}_{21} \mathrm{H}_{23} \mathrm{O}_{6}, 371.1496\right)$; EIMS $(70 \mathrm{eV}) \mathrm{m} / \mathrm{z}$ (rel. int.): $370\left(\mathrm{M}^{+}, 22\right), 329(80), 287(12), 269(9), 208(100)$, 149(82), 137(73), 77(31).

(7S,8R, 1'R, 3'R)-4'-Hydroxy-3,4-methylenedioxy-3',5',5trimethoxy-2',4'-dioxo- $\Delta^{1,3,5,5,8^{\prime}}$-8.1',7.3'-neolignan (5e)

Viscous oil; $[\alpha]_{D}^{21}=-29^{\circ}(\mathrm{MeOH}, c=4.48 \mathrm{~g} / 100 \mathrm{~mL})$; ${ }^{1} \mathrm{H}$ and ${ }^{13} \mathrm{C}$ NMR, see Table 2; EIMS (70 eV) $\mathrm{m} / \mathrm{z}$ (rel. int.): 400 ( $\left.\mathrm{M}^{+}, 52\right), 359(21), 331(10), 288(14), 219(100)$, 208(45), 207(43), 193(18), 192(66), 180(53), 165(34), 137(23), 91(14), 77(16).

\section{$7 \boldsymbol{a}$ (Dimer of $\mathbf{5 c}$ )}

Viscous oil; $[\alpha]_{D}^{21}=+220^{\circ}(\mathrm{MeOH}, c=0.07 \mathrm{~g} / 100 \mathrm{~mL})$; IR (film) $v_{\max } / \mathrm{cm}^{-1}: 2922,1766,1714,1513,1453,1137$, 1094,1044; ${ }^{1} \mathrm{H}$ and ${ }^{13} \mathrm{C}$ NMR, see Table 2; CIMS $\mathrm{m} / \mathrm{z}$ (rel. int.): 741( $\left.\mathrm{M}^{+}, 1\right)$, 579(1), 419(15), 391(91), 371(100), 341(18), 209(28), 163(37), 57(27).

\section{$7 \boldsymbol{b}$ (Dimer of $5 \boldsymbol{e})$}

Viscous oil; $[\alpha]_{D}^{21}=+57^{\circ}(\mathrm{MeOH}, c=0.62 \mathrm{~g} / 100 \mathrm{~mL})$; IR (film) $v_{\max } / \mathrm{cm}^{-1}: 1767,1714,1505,1491,1445,1240$, 1039; ${ }^{1} \mathrm{H}$ and ${ }^{13} \mathrm{C}$ NMR, see Table 2; CIMS $m / z$ (rel. int.): 801( $\left.\mathrm{M}^{+}, 1\right), 609(18), 429(76), 419(85), 401(100), 371(11)$, 209(17), 193(11).

rel(8R)-Humulan-1,4-dien-8-ol (9)

Solid amorphous. Found: C, $80.89 \%, \mathrm{H}, 11.65 \%$ $\left(\mathrm{C}_{15} \mathrm{H}_{26} \mathrm{O}\right.$ requires: $\left.\mathrm{C}, 81.02 \%, \mathrm{H}, 11.79 \%\right)$; IR (film) $V_{\max } / \mathrm{cm}^{-1}: 3425,2946,1707,1446,1371,1022,762 ;{ }^{1} \mathrm{H}$ and ${ }^{13} \mathrm{C}$ NMR, see Table 3; EIMS (70 eV) $\mathrm{m} / z$ (rel. int.): 344( $\left.\mathrm{M}^{+}, 27\right), 303(9), 271(12), 189(100), 179(26), 178(76)$, 166(25), 165(66), 151(59), 137(19), 115(15), 107(27), 91(35), 77(33).

\section{Acknowledgments}

The authors acknowledge FAPESP, CNPq, CAPES and International Foundation for Science (Sweden) for financial support.

\section{Supplementary Information}

Supplementary information is available free of charge at http://jbcs.org.br, as PDF file.

\section{References}

1. Santa-Catarina, C.; Olmedo, A. S.; Meyer, G. A.; Macedo, J.; Amorin, W.; Viana, A. M.; Plant Cell Tiss. Organ Cult. 2003, $78,55$.

2. Viana, A. M.; Mantell, S.H. In Somatic Embryogenesis in Woody Plants; Jain, S.M.; Gupta, P.K.; Newton, R.J. eds.; Kluwer Academic Publishers: Dordrecht, 1999, Vol. 5, pp. 3-30.

3. Gottlieb, O. R.; Phytochemistry 1972, 11, 1537.

4. Lordello, A.L.L.; Yoshida, M.; Phytochemistry 1997, 46, 741.

5. Haraguchi, M.; Motidome, M.; Yoshida, M.; Gottlieb, O.R.; Phytochemistry 1983, 22, 561.

6. Ishige, M.; Motidome, M.; Yoshida, M.; Gottlieb, O.R.; Phytochemistry 1991, 30, 4121.

7. Dias, D.A.; Yoshida, M.; Gottlieb, O.R.; Phytochemistry 1986, 25, 2613.

8. Marques, M.O.M.; Gomes, M.C.C.P.; Yoshida, M.; Gottlieb, O.R.; Phytochemistry 1992, 31, 275.

9. David, J.M.; Yoshida, M.; Gottlieb, O.R.; Phytochemistry 1994, 36, 491.

10. Felício, J.D.; Motidome, M.; Yoshida, M.; Gottlieb, O.R.; Phytochemistry 1986, 25, 1707.

11. Romoff, P.; Yoshida, M.; Gottlieb, O.R.; Phytochemistry 1984, 23, 2101.

12. Rodrigues, D.C.; Yoshida, M.; Gottlieb, O.R.; Phytochemistry 1992, 31, 271.

13. Alvarenga, M.A.; Castro, O.; Giesbrecht, A.M.; Gottlieb, O.R.; Phytochemistry 1977, 16, 1801.

14. Iwabuchi, H.; Yoshikura, M.; Kamisako, W.; Chem. Pharm. Bull. 1989, 37, 509.

15. Dodson, C.D.; Stermitz, F.R.; Castro, O.; Janzen, D.H.; Phytochemistry 1987, 26, 2037.

16. Carvalho, M.G.; Yoshida, M.; Gottlieb, O.R.; Gottlieb, H.E.; Phytochemistry 1988, 27, 2319.

17. Randriamiharisoa, R.; Gaydou, E.M.; Faure, R.; Bianchini, J.P.; Magn. Reson. Chem. 1986, 24, 275.

18. Santa-Catarina, C.; Randi, A.M.; Viana, A.M.; Plant Cell, Tissue Organ Cult. 2003, 74, 67.

Received: October 1, 2008 Web Release Date: March 20, 2009

FAPESP helped in meeting the publication costs of this article. 


\section{Neolignans and Sesquiterpenes from Leaves and Embryogenic Cultures of Ocotea catharinensis (Lauraceae)}

Mariko Funasaki, ${ }^{a}$ Ana Luísa L. Lordello, ${ }^{b}$ Ana Maria Viana, ${ }^{c}$ Claudete Santa-Catarina, ${ }^{d}$ Eny I. S. Floh, ${ }^{d}$ Massayoshi Yoshida ${ }^{a, e}$ and Massuo J. Kato ${ }^{*, a}$

${ }^{a}$ Instituto de Química, Universidade de São Paulo, CP 26077, 05513-970 São Paulo-SP, Brazil

${ }^{b}$ Departamento de Química, Universidade Federal do Paraná, CP 19081, 81531-990 Curitiba-PR, Brazil

'Departamento de Botânica, Centro de Ciências Biológicas, Universidade Federal de Santa Catarina, 88040-900 Florianópolis - SC, Brazil

${ }^{d}$ Instituto de Biociências, Universidade de São Paulo, CP 11461, 05508-900 São Paulo-SP, Brazil

${ }^{e}$ Centro de Biotecnologia da Amazônia, Av. Gov. Danilo de Matos Areosa, 690, 69075-351 Manaus-AM, Brazil
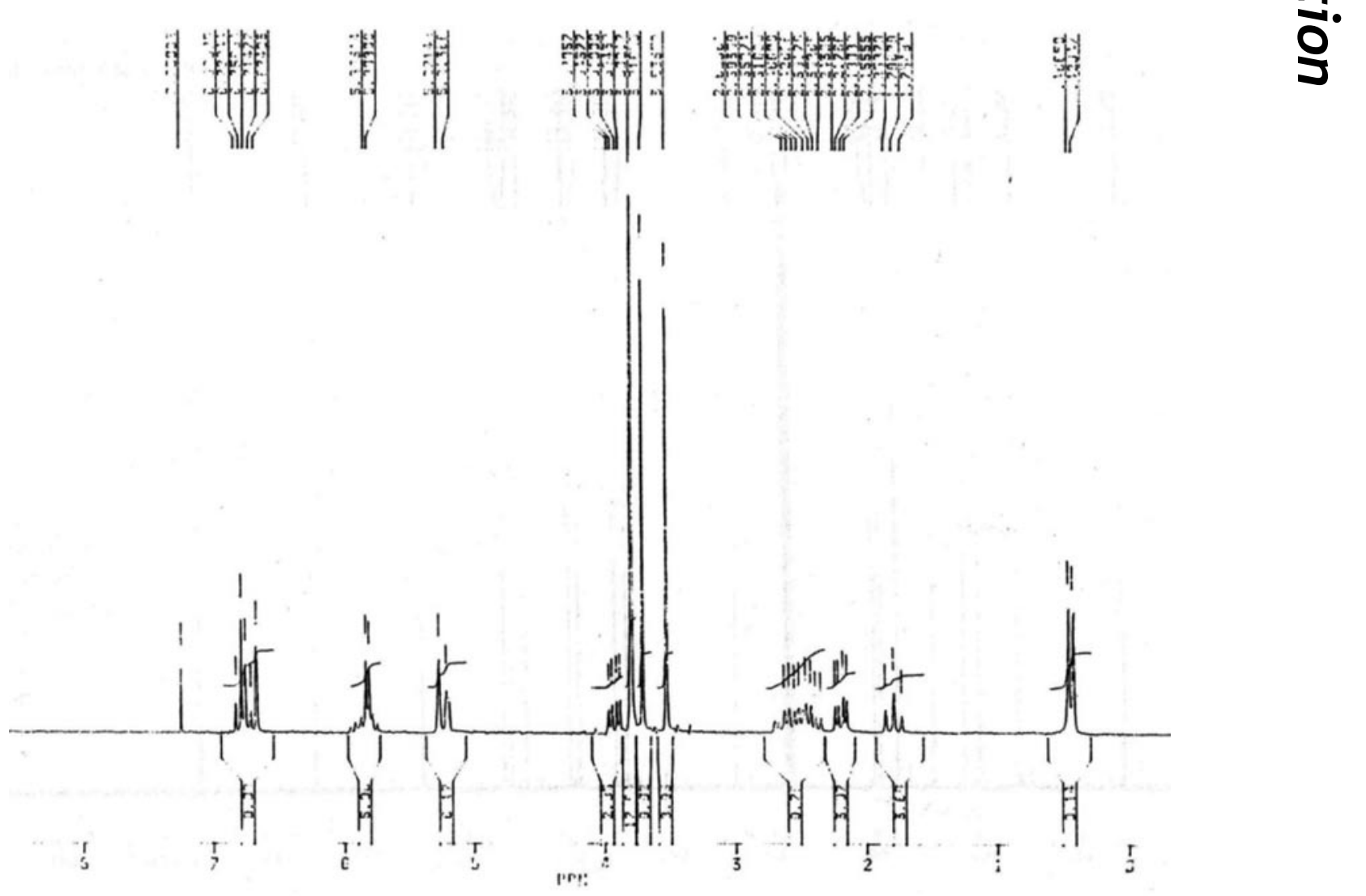

Figure S1. ${ }^{1} \mathrm{H}$ NMR spectrum of $\mathbf{1 a}\left(200 \mathrm{MHz}, \mathrm{CDCl}_{3}\right)$. 


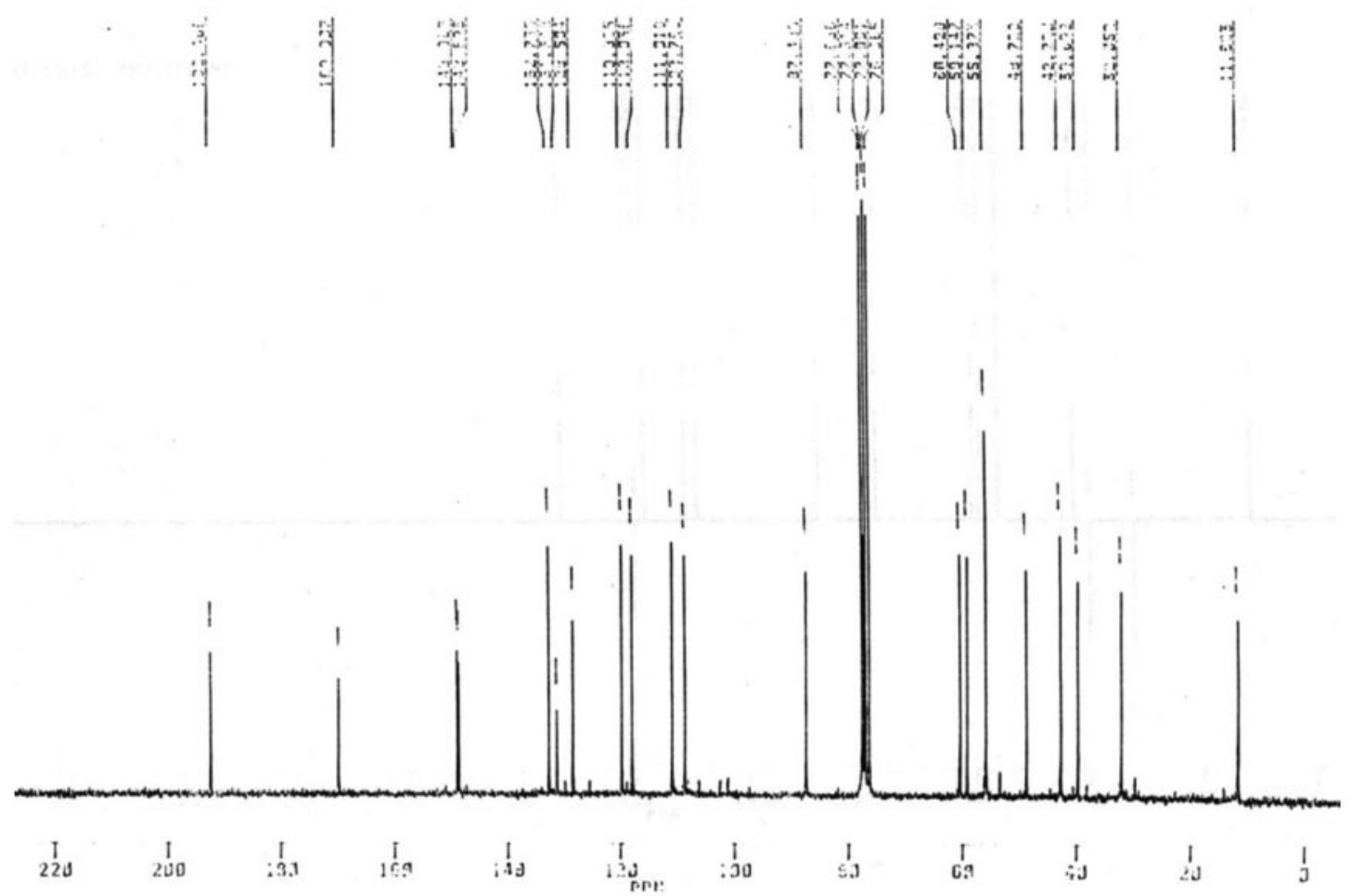

Figure S2. ${ }^{13} \mathrm{C}$ NMR spectrum of $1 \mathrm{a}\left(50 \mathrm{MHz}, \mathrm{CDCl}_{3}\right)$.

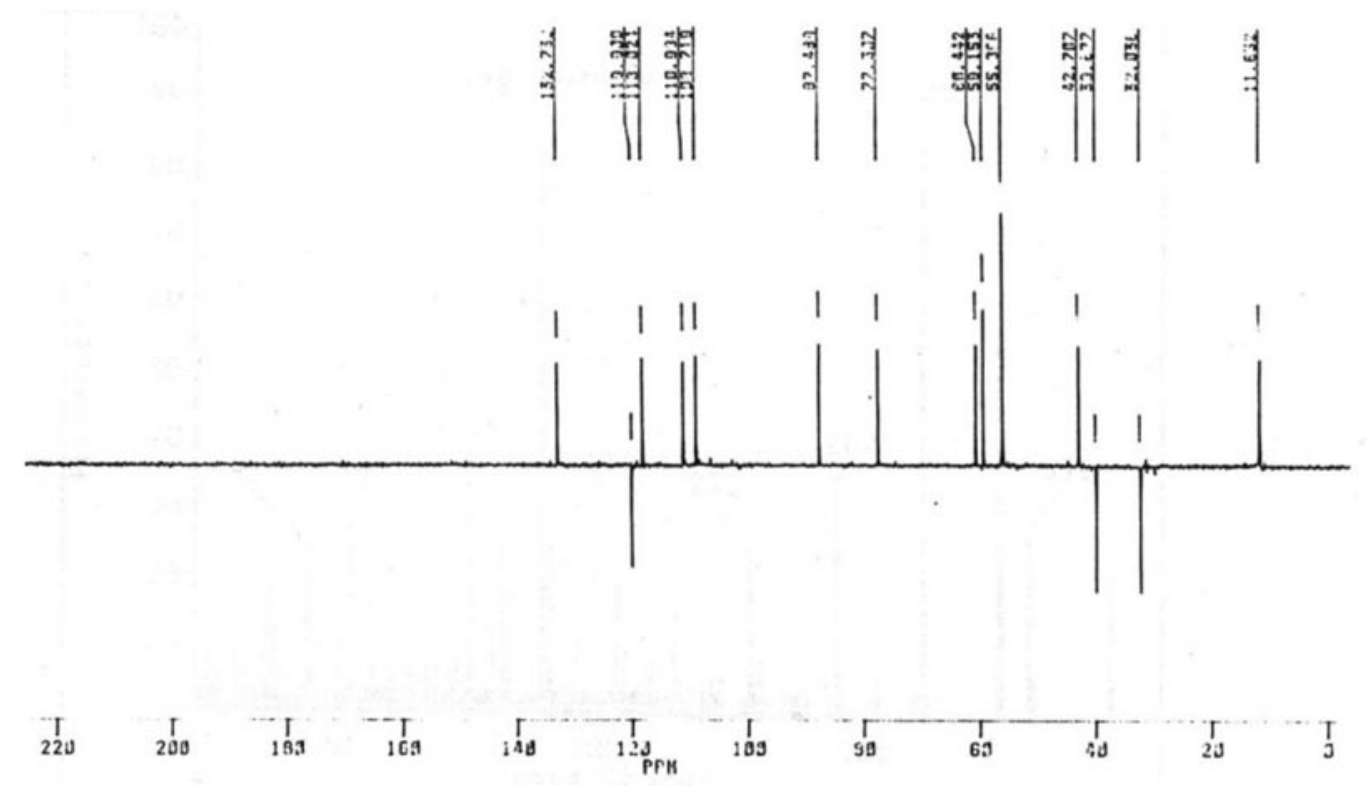

Figure S3. DEPT $135{ }^{13} \mathrm{C}$ NMR spectrum of $\mathbf{1 a}\left(50 \mathrm{MHz}, \mathrm{CDCl}_{3}\right)$. 


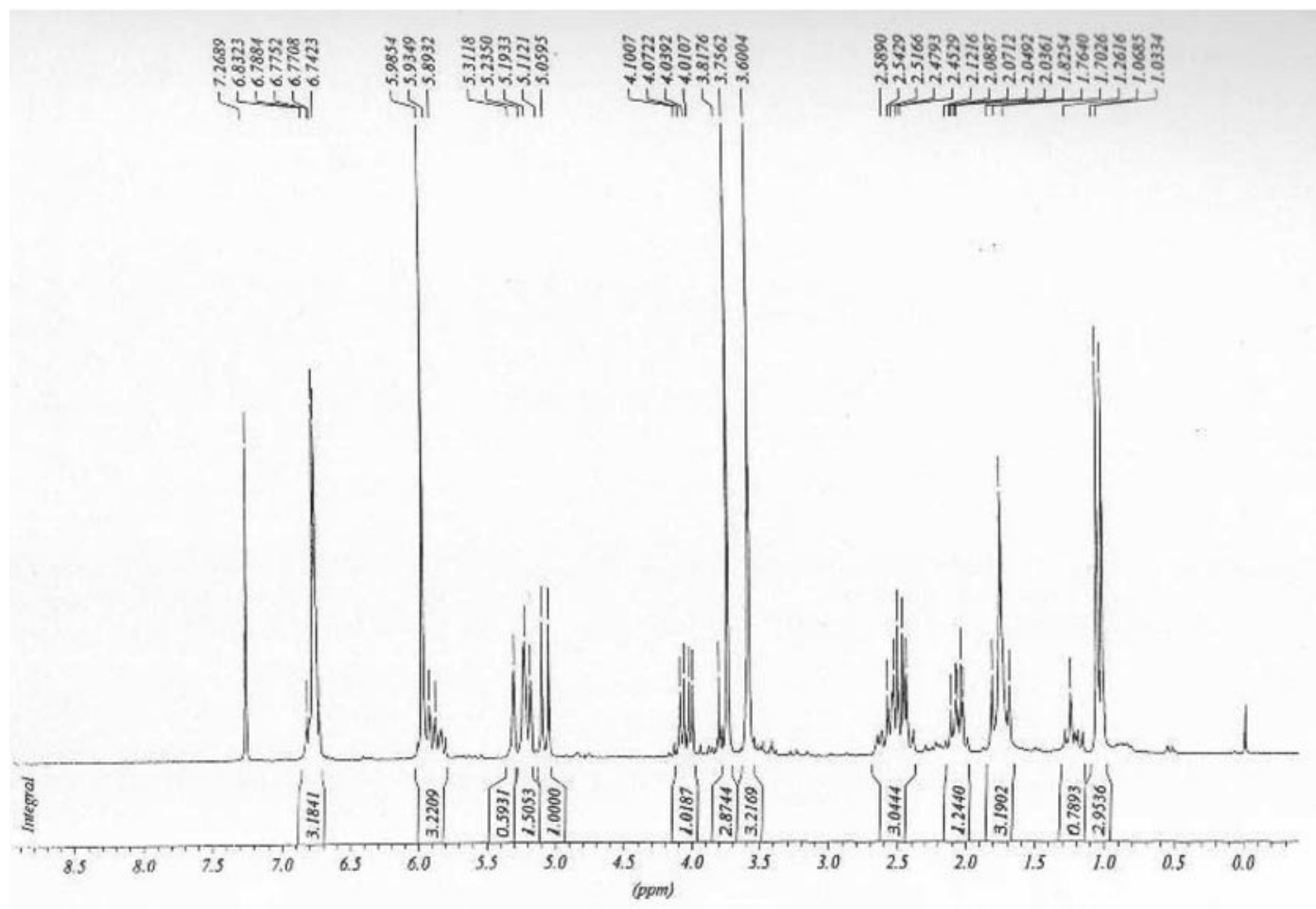

Figure S4. ${ }^{1} \mathrm{H}$ NMR spectrum of $\mathbf{1 b}\left(200 \mathrm{MHz}, \mathrm{CDCl}_{3}\right)$.

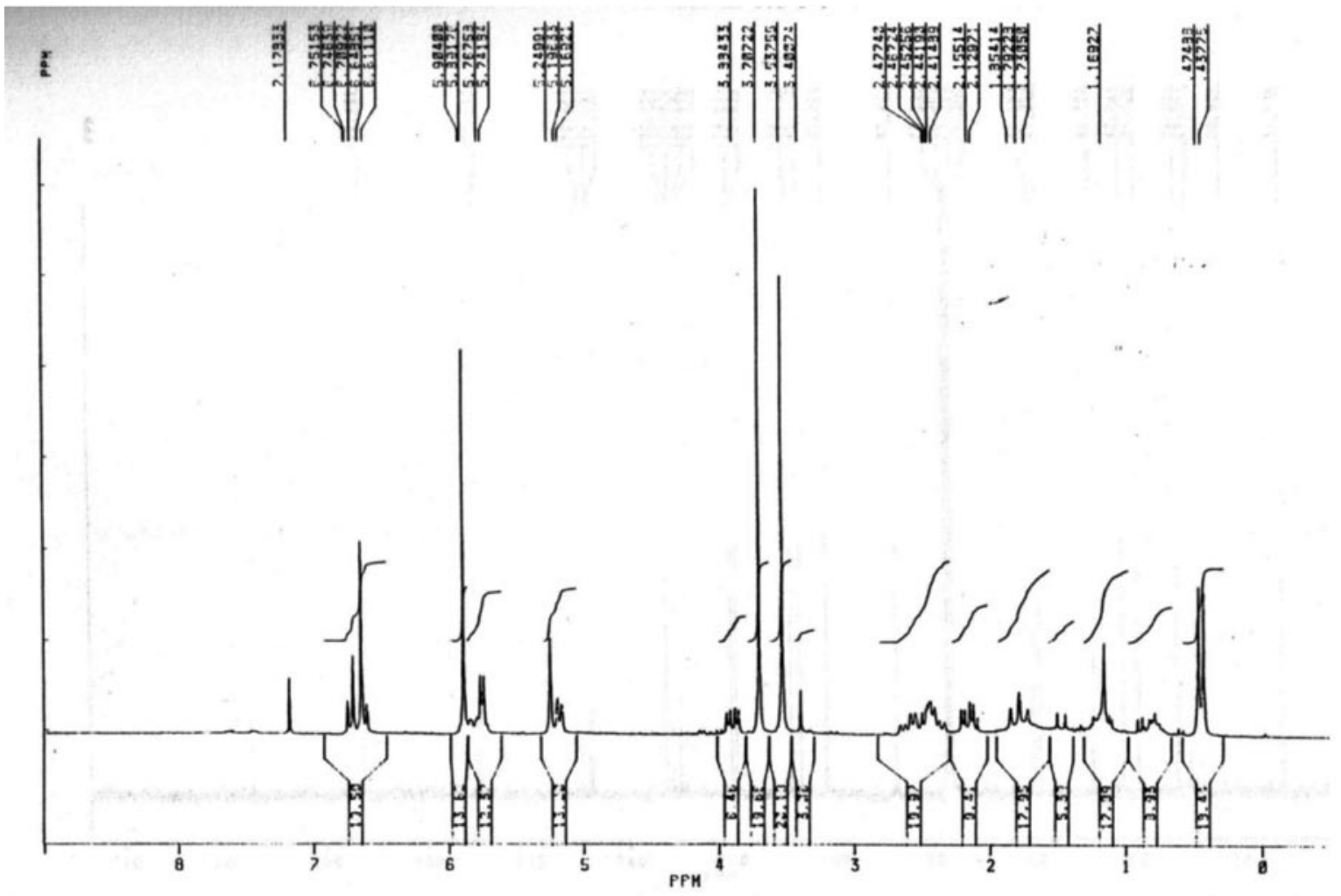

Figure S5. ${ }^{1} \mathrm{H}$ NMR spectrum of $1 \mathrm{c}\left(200 \mathrm{MHz}, \mathrm{CDCl}_{3}\right)$. 


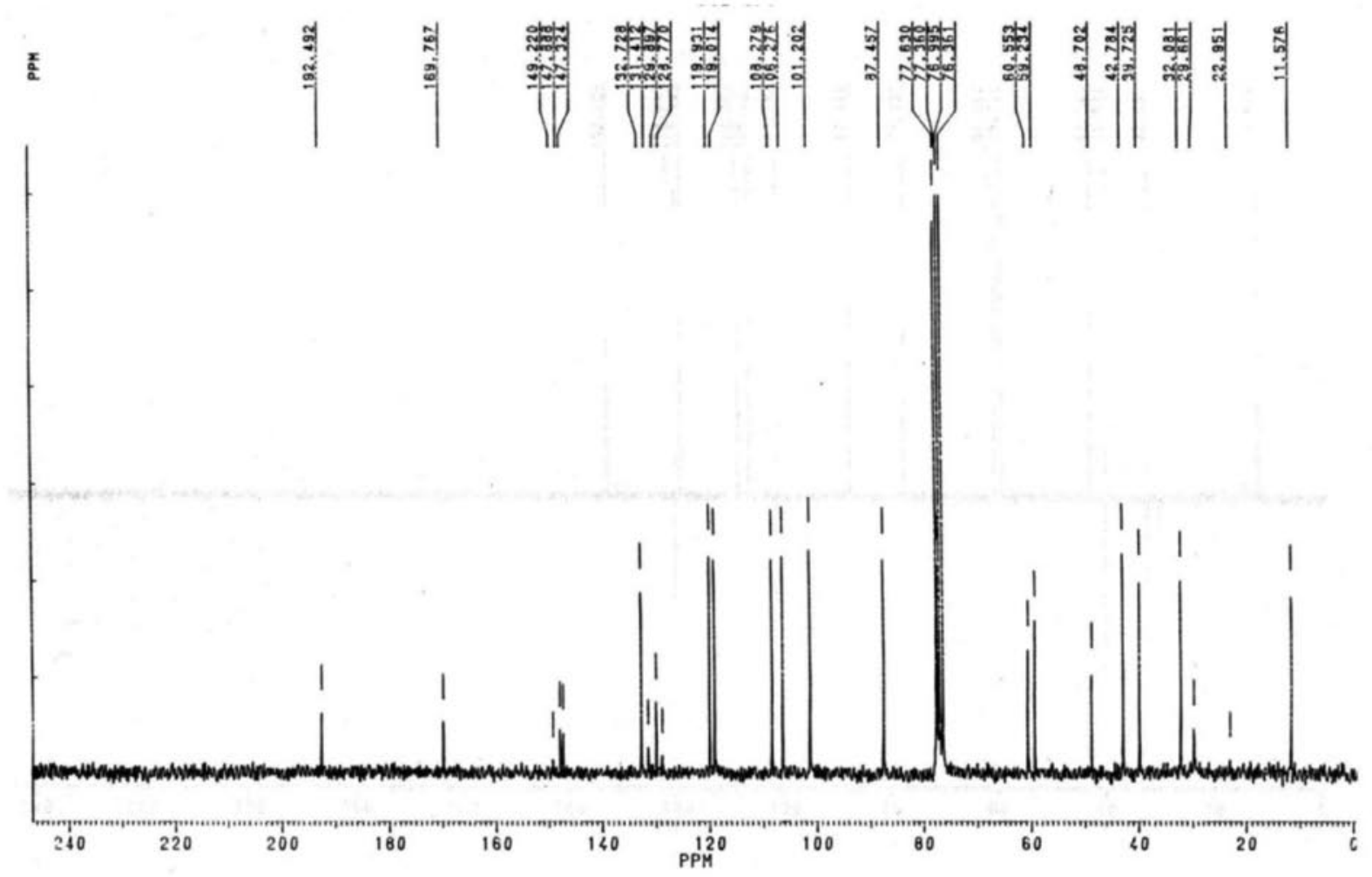

Figure S6. ${ }^{13} \mathrm{C}$ NMR spectrum of $1 \mathbf{c}\left(50 \mathrm{MHz}, \mathrm{CDCl}_{3}\right)$.

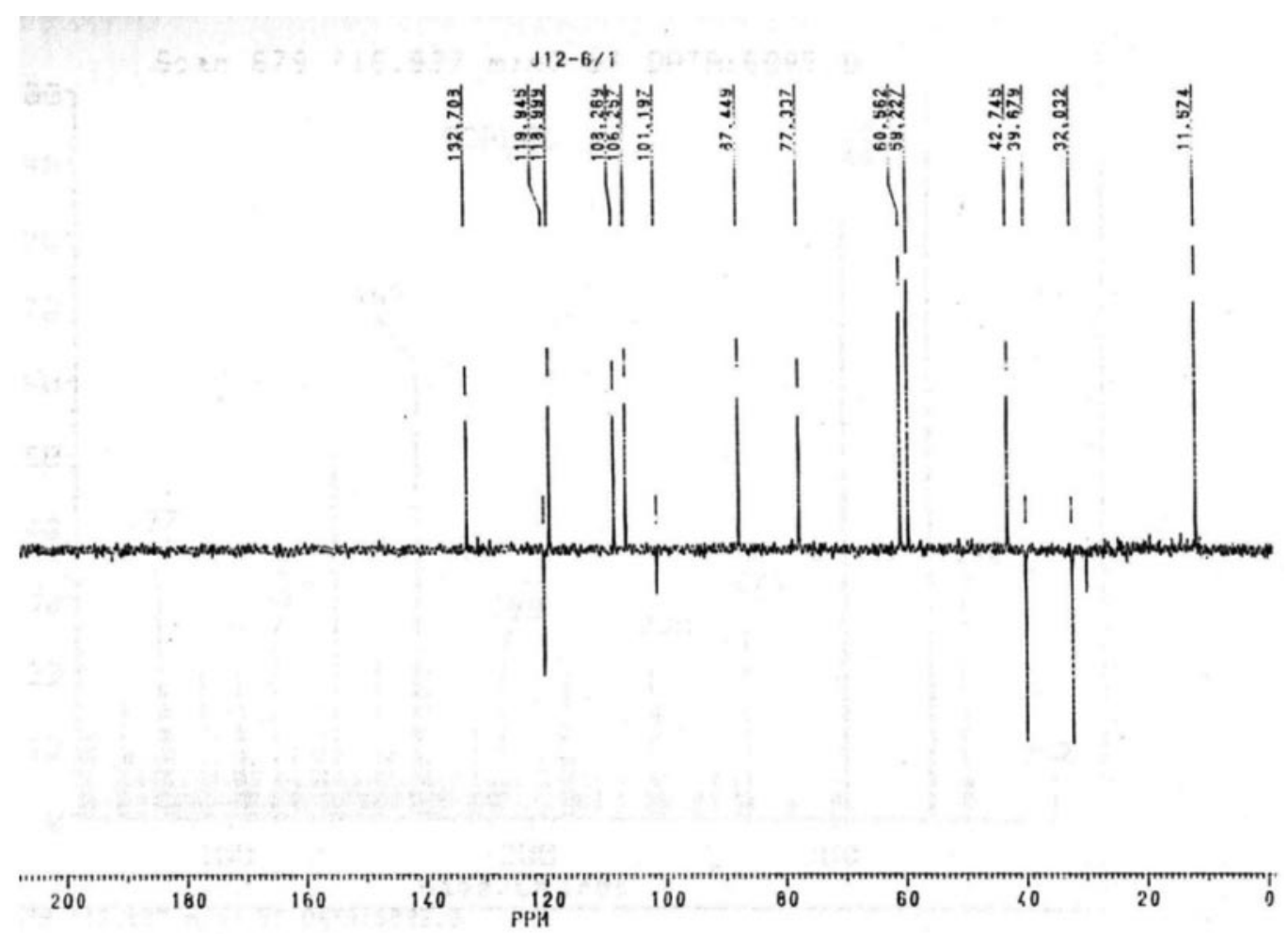

Figure S7. DEPT $135{ }^{13} \mathrm{C}$ NMR spectrum of $1 \mathbf{c}\left(50 \mathrm{MHz}, \mathrm{CDCl}_{3}\right)$. 


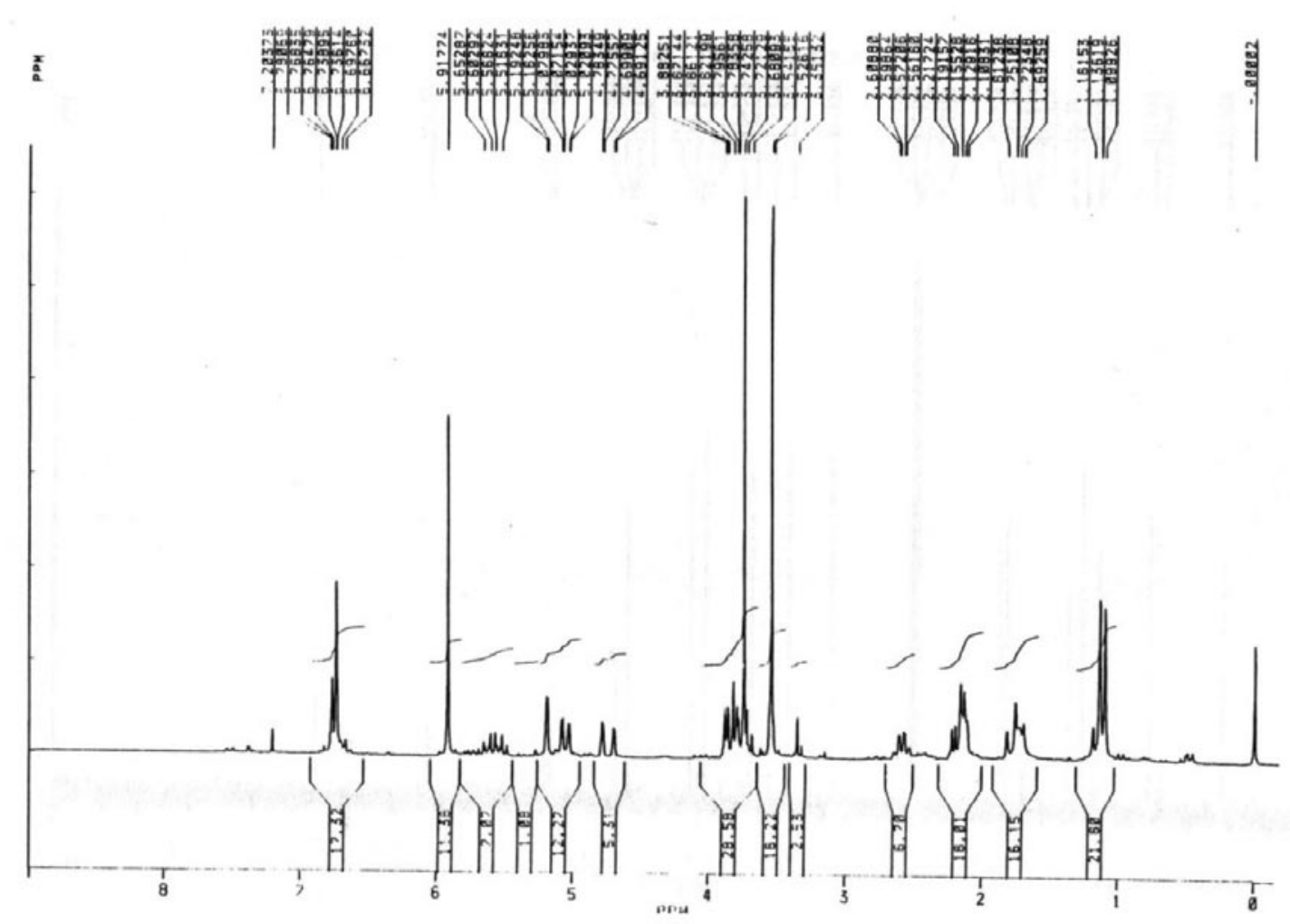

Figure S8. ${ }^{1} \mathrm{H}$ NMR spectrum of $\mathbf{1 e}\left(200 \mathrm{MHz}, \mathrm{CDCl}_{3}\right)$.

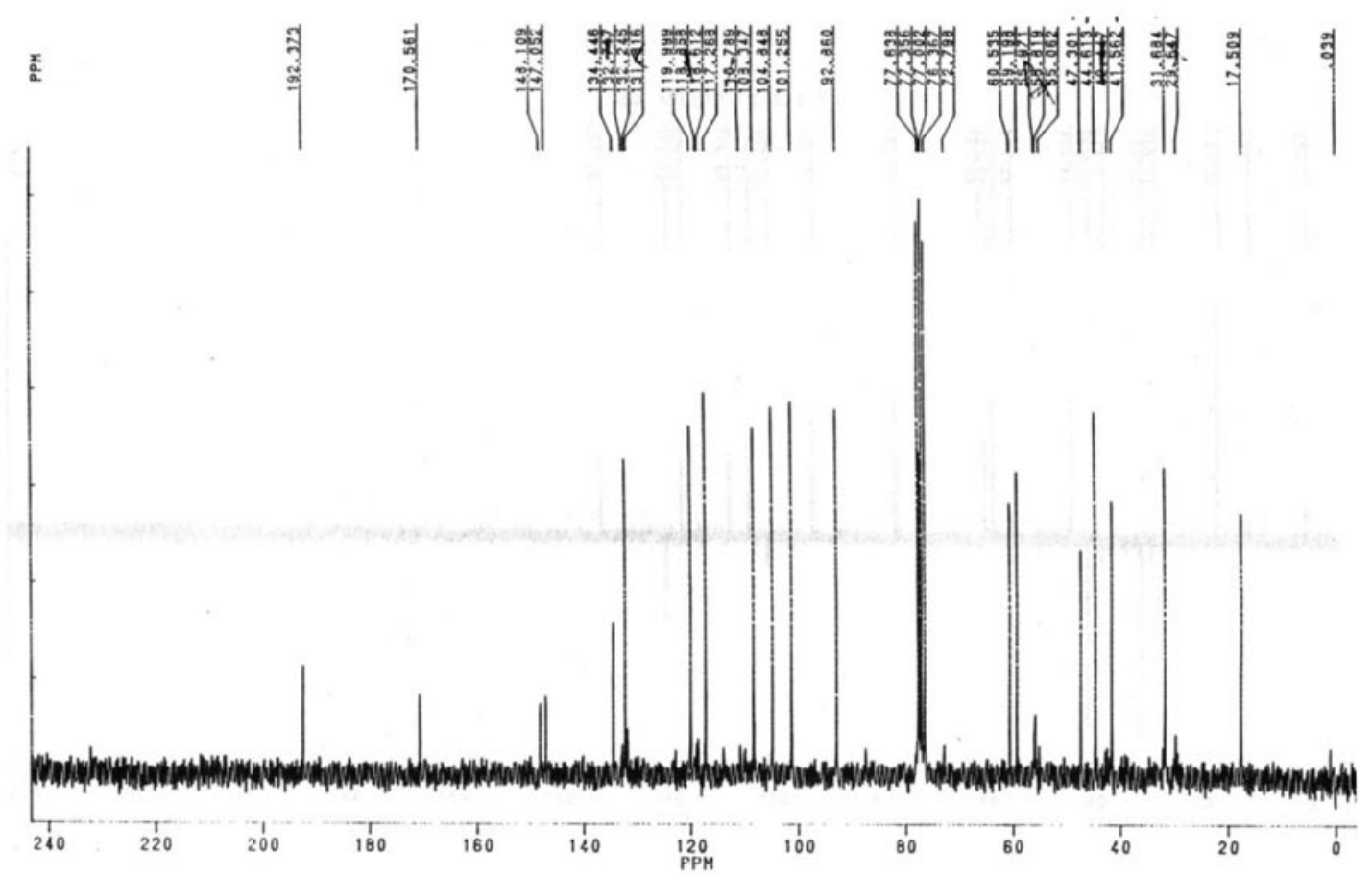

Figure S9. ${ }^{13} \mathrm{C} \mathrm{NMR}$ spectrum of $\mathbf{1 e}\left(50 \mathrm{MHz}, \mathrm{CDCl}_{3}\right)$. 

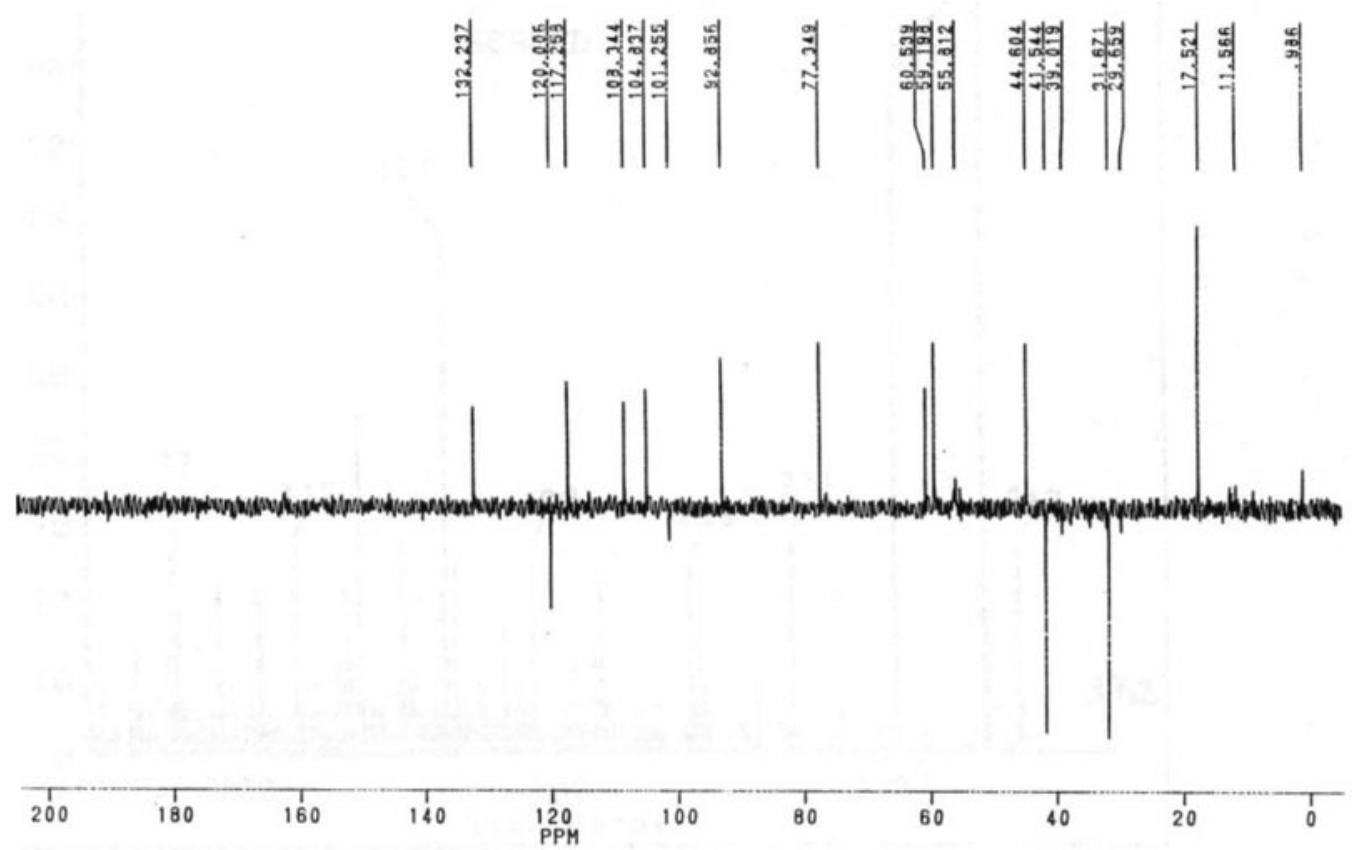

Figure S10. DEPT $135{ }^{13} \mathrm{C}$ NMR spectrum of $1 \mathrm{e}\left(50 \mathrm{MHz}, \mathrm{CDCl}_{3}\right)$.

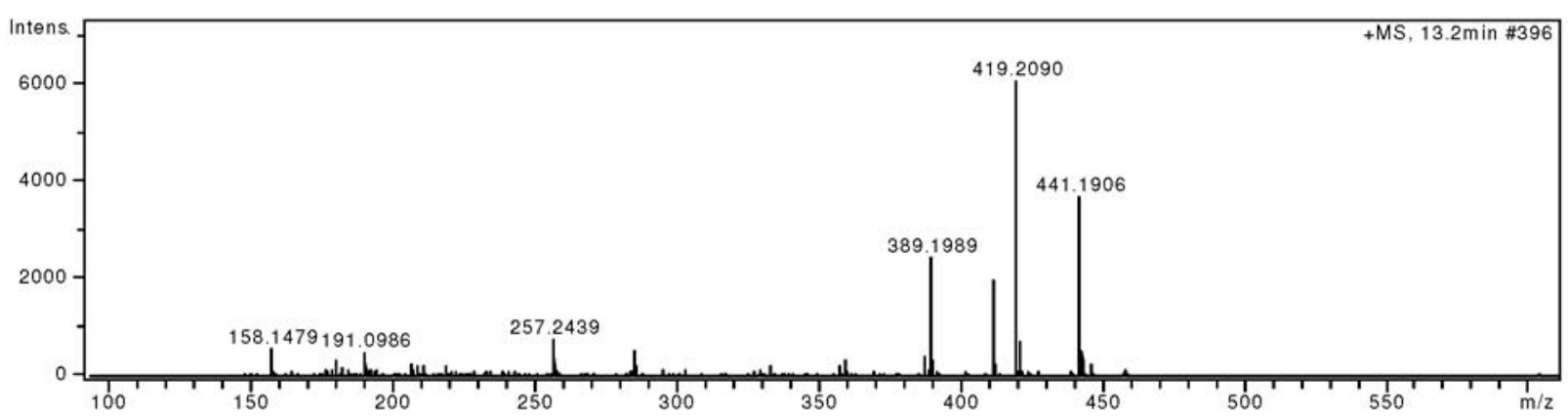

Figure S11. HRESIMS spectrum of 1e. 


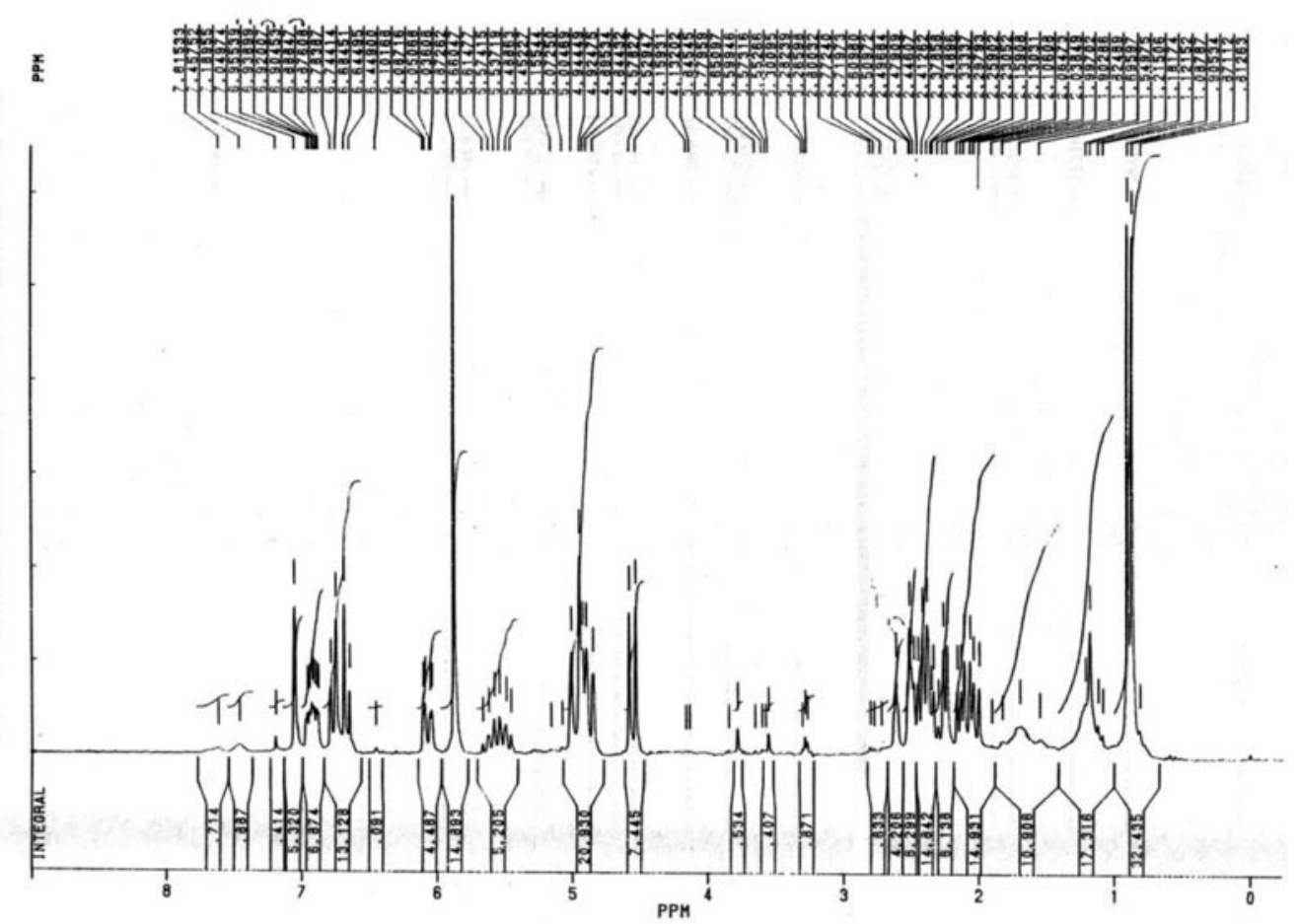

Figure S12. ${ }^{1} \mathrm{H}$ NMR spectrum of $\mathbf{2 a}\left(200 \mathrm{MHz}, \mathrm{CDCl}_{3}\right)$.

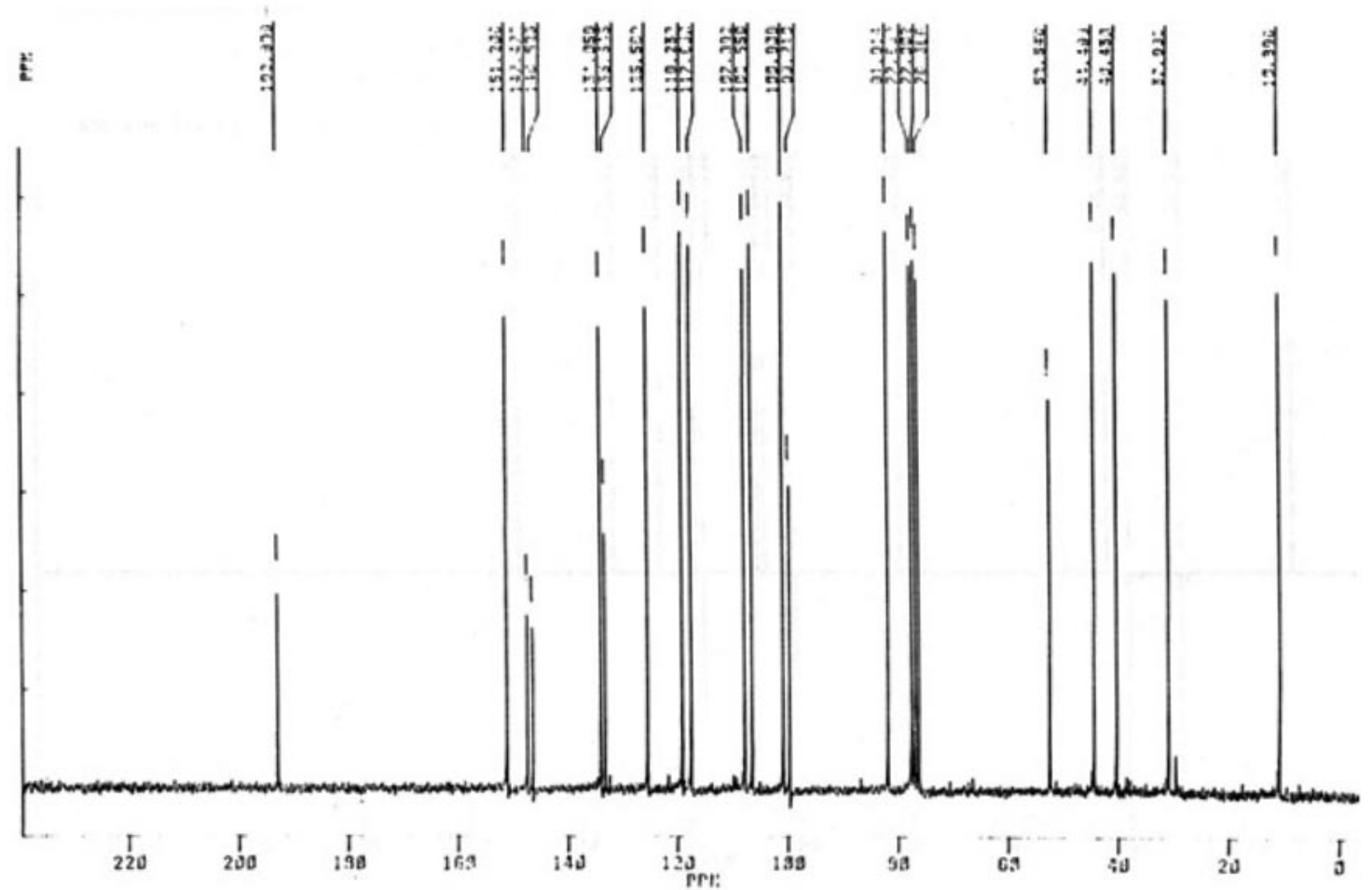

Figure S13. ${ }^{13} \mathrm{C}$ NMR spectrum of $\mathbf{2 a}\left(50 \mathrm{MHz}, \mathrm{CDCl}_{3}\right)$. 


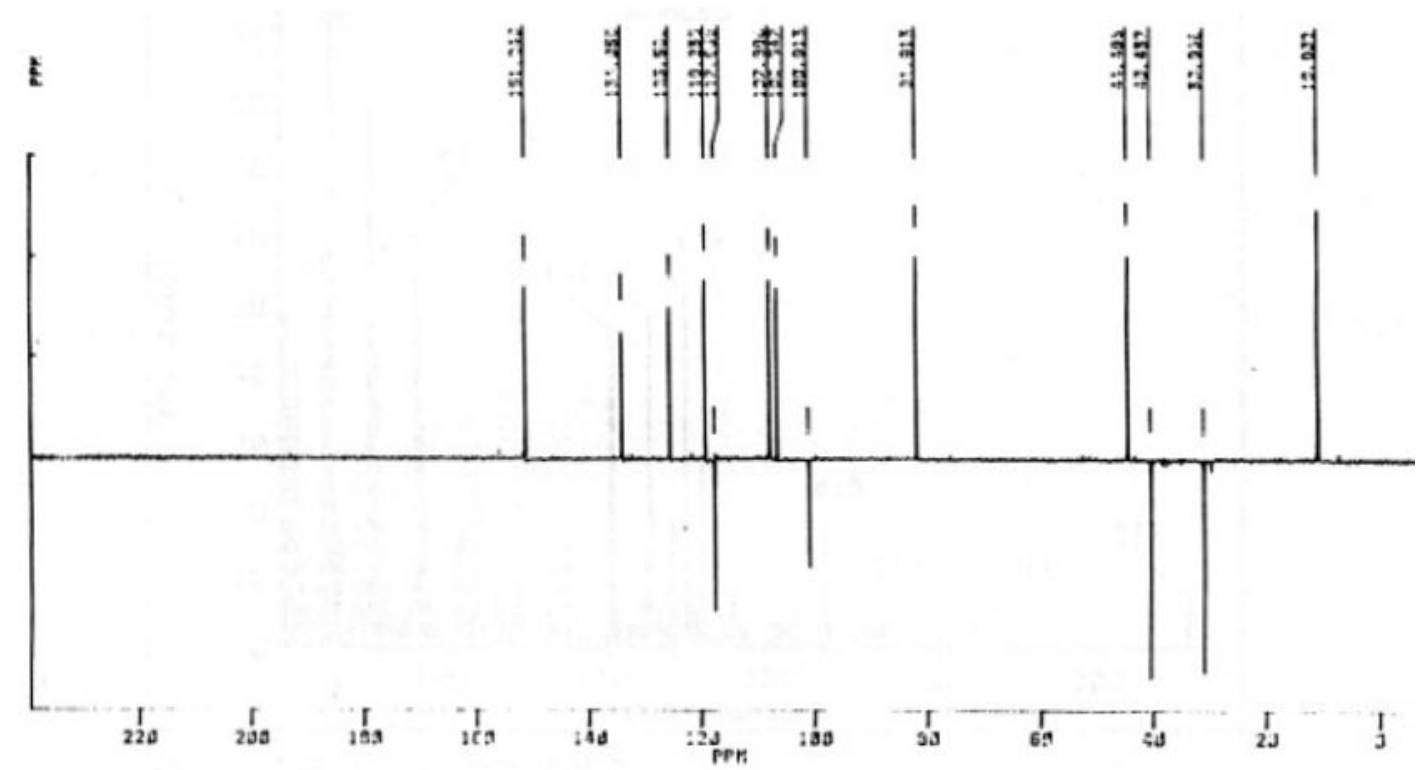

Figure S14. DEPT $135{ }^{13} \mathrm{C}$ NMR spectrum of $2 \mathbf{a}\left(50 \mathrm{MHz}, \mathrm{CDCl}_{3}\right)$.

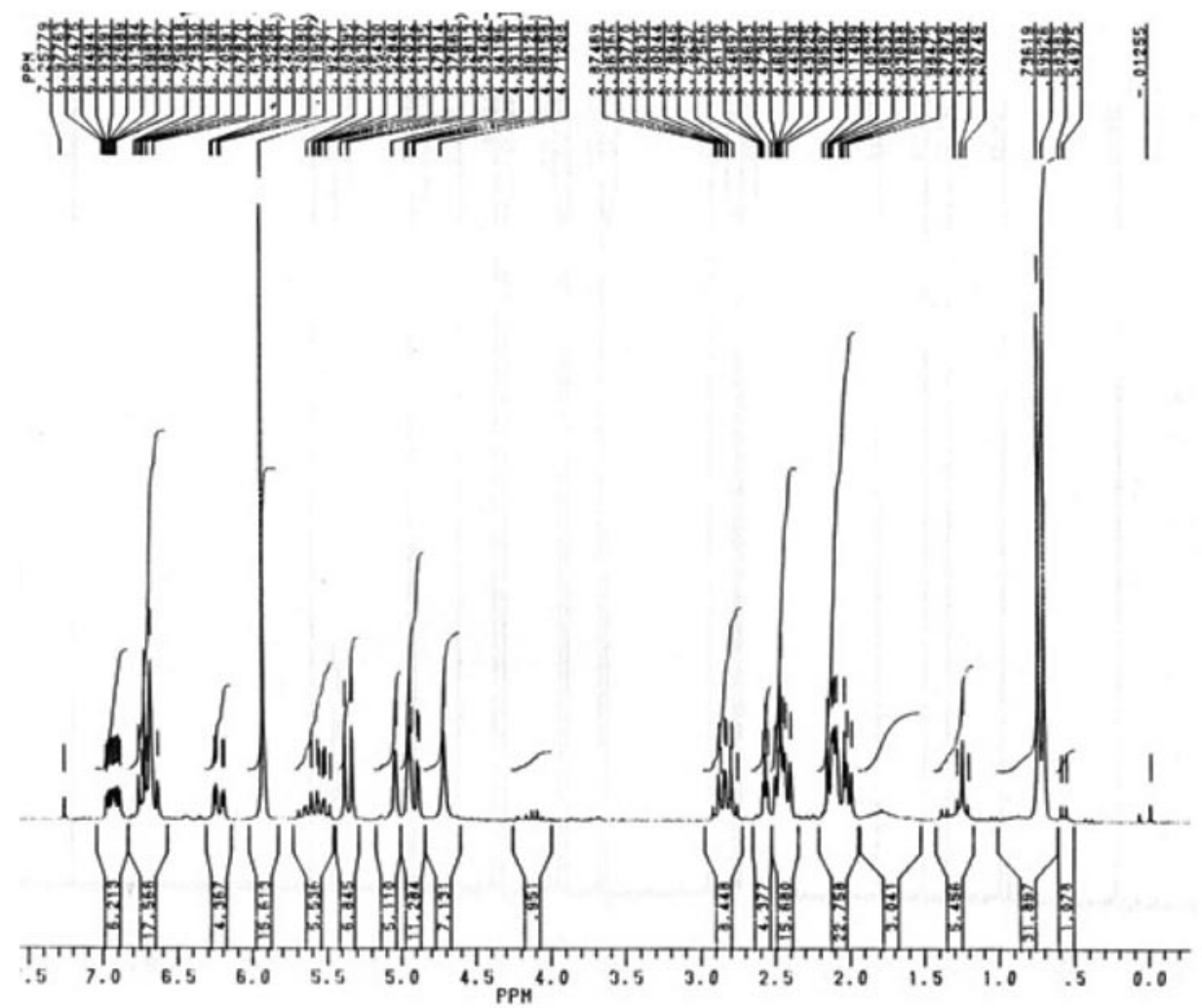

Figure S15. ${ }^{1} \mathrm{H}$ NMR spectrum of $\mathbf{2 b}\left(200 \mathrm{MHz}, \mathrm{CDCl}_{3}\right)$. 


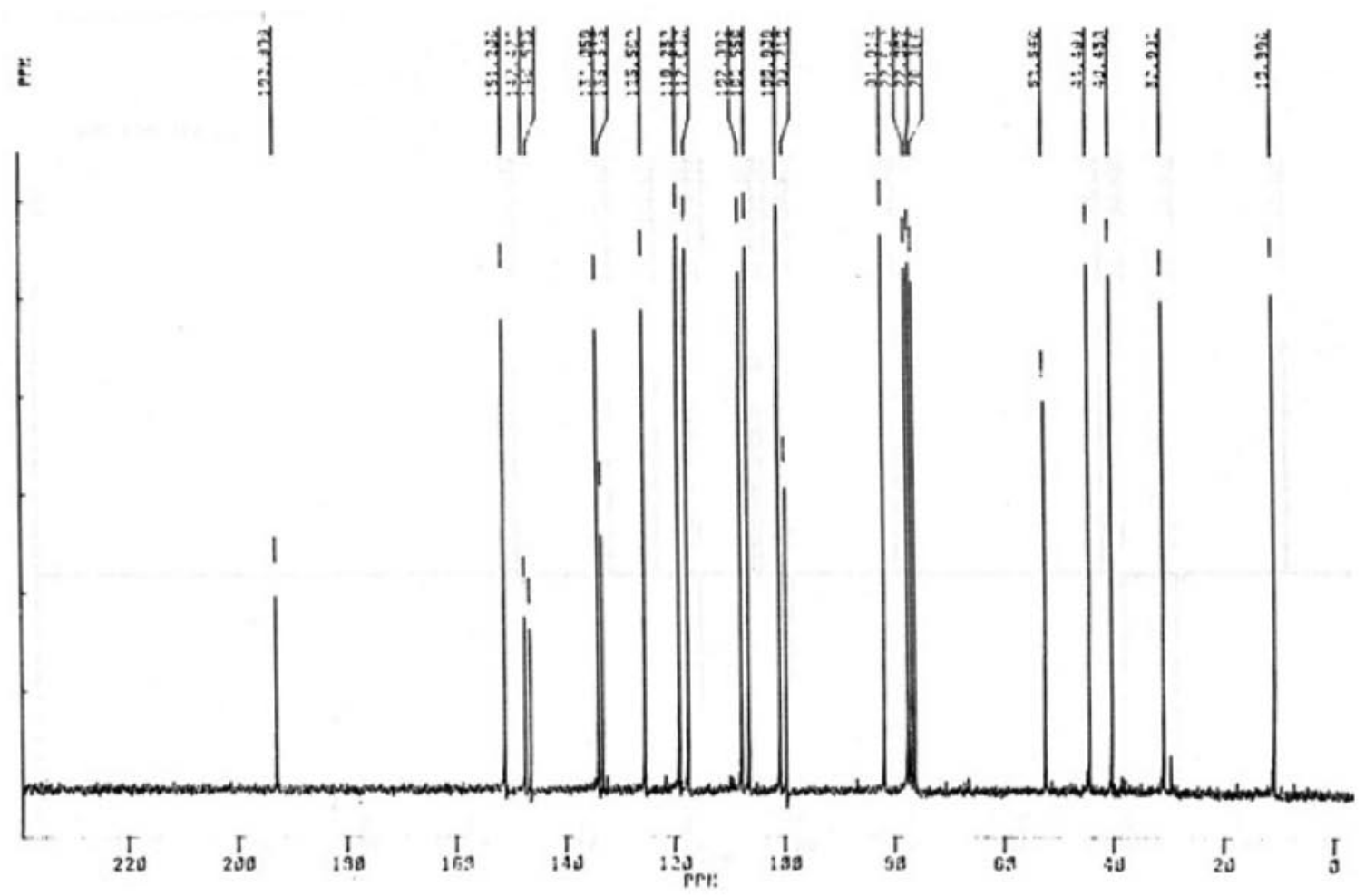

Figure S16. ${ }^{13} \mathrm{C}$ NMR spectrum of $\mathbf{2 b}\left(50 \mathrm{MHz}, \mathrm{CDCl}_{3}\right)$.

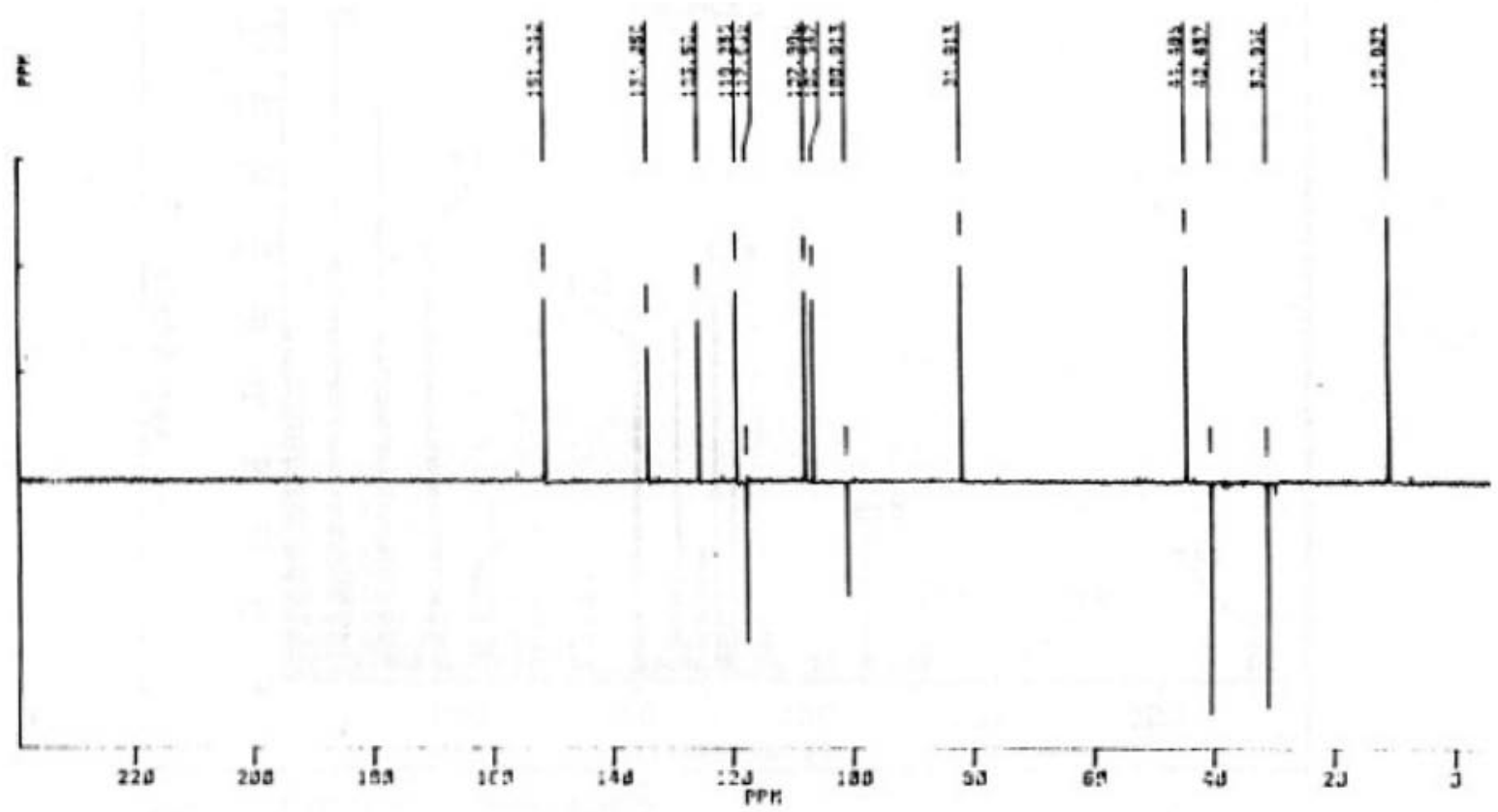

Figure S17. DEPT $135{ }^{13} \mathrm{C}$ NMR spectrum of $\mathbf{2 b}\left(50 \mathrm{MHz}, \mathrm{CDCl}_{3}\right)$. 


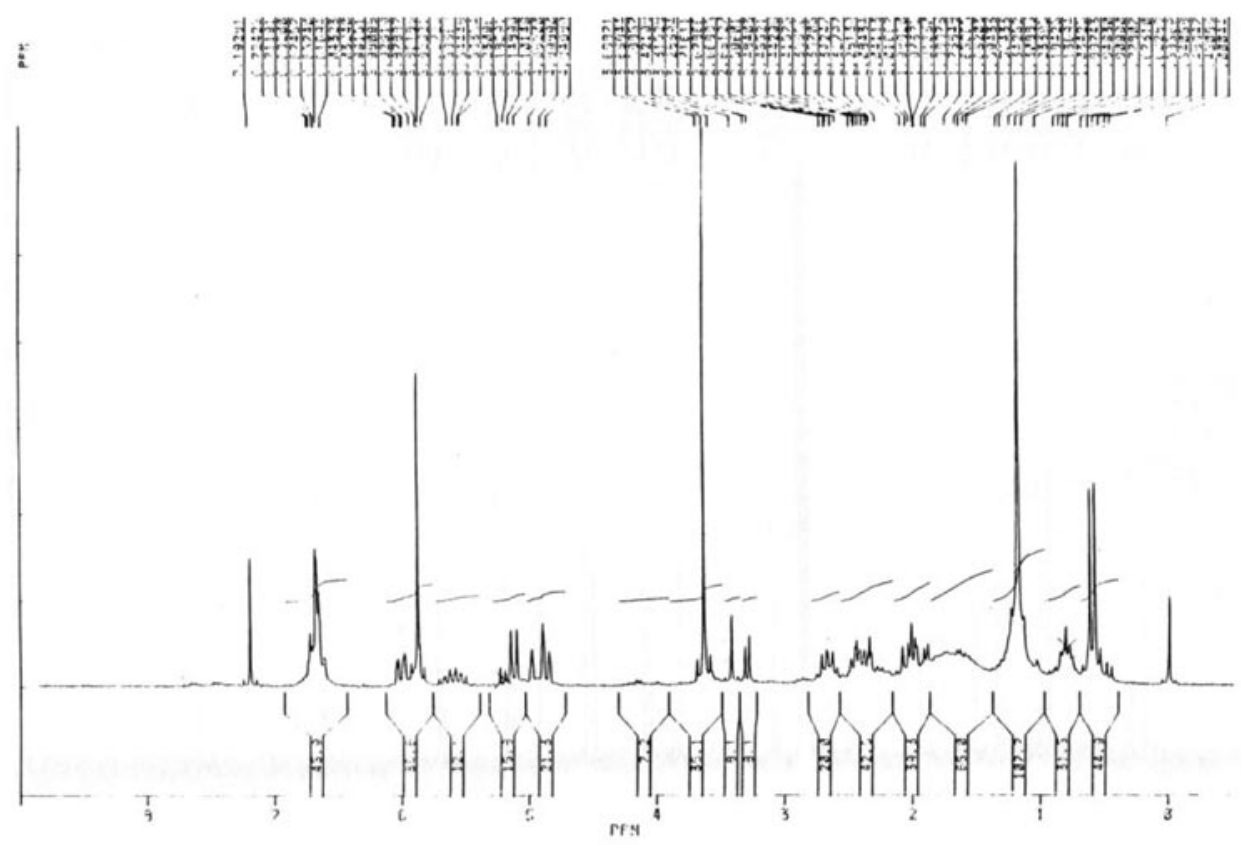

Figure S18. ${ }^{1} \mathrm{H}$ NMR spectrum of $\mathbf{2 c}\left(200 \mathrm{MHz}, \mathrm{CDCl}_{3}\right)$.

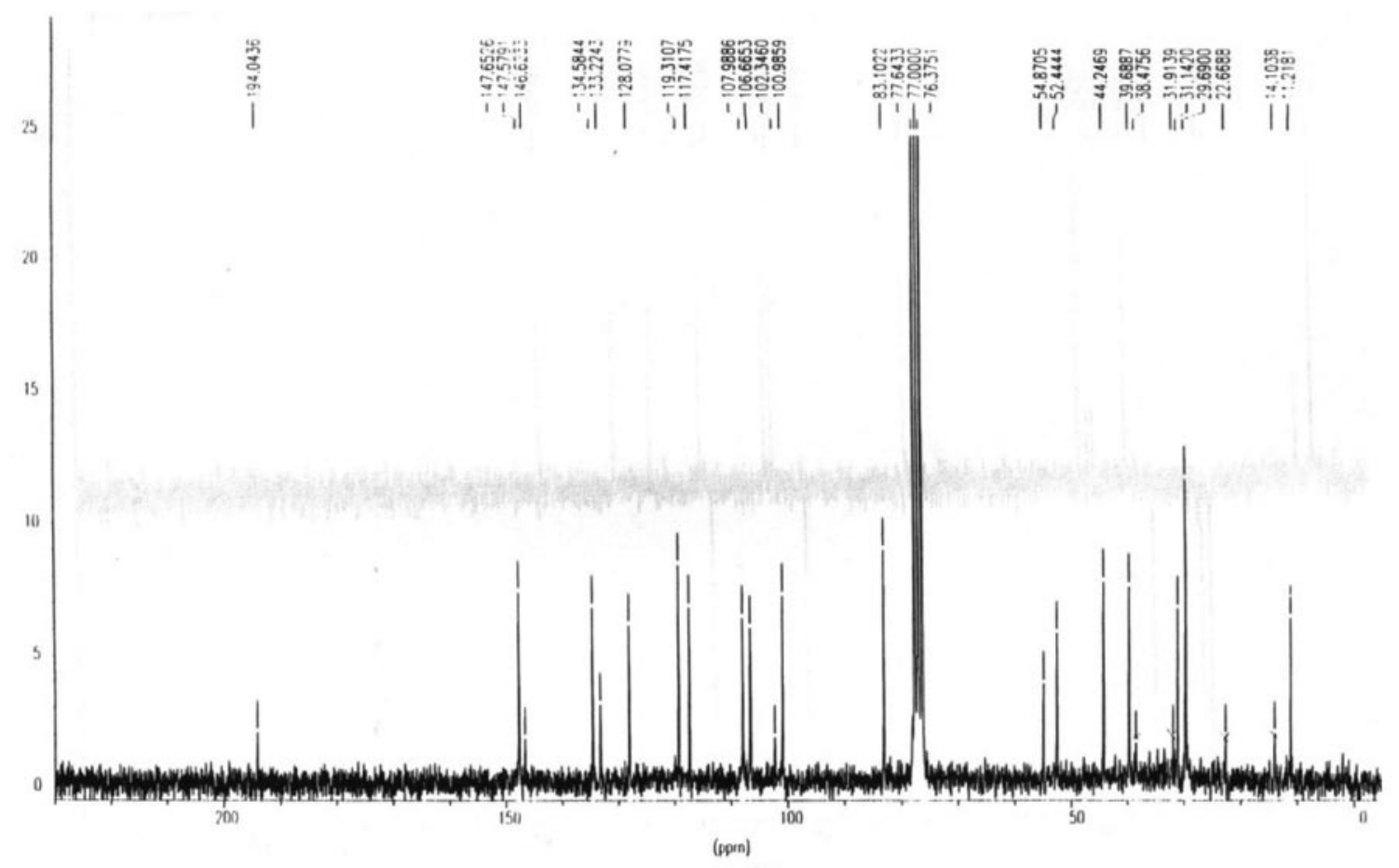

Figure S19. ${ }^{13} \mathrm{C}$ NMR spectrum of $2 \mathrm{c}\left(50 \mathrm{MHz}, \mathrm{CDCl}_{3}\right)$. 


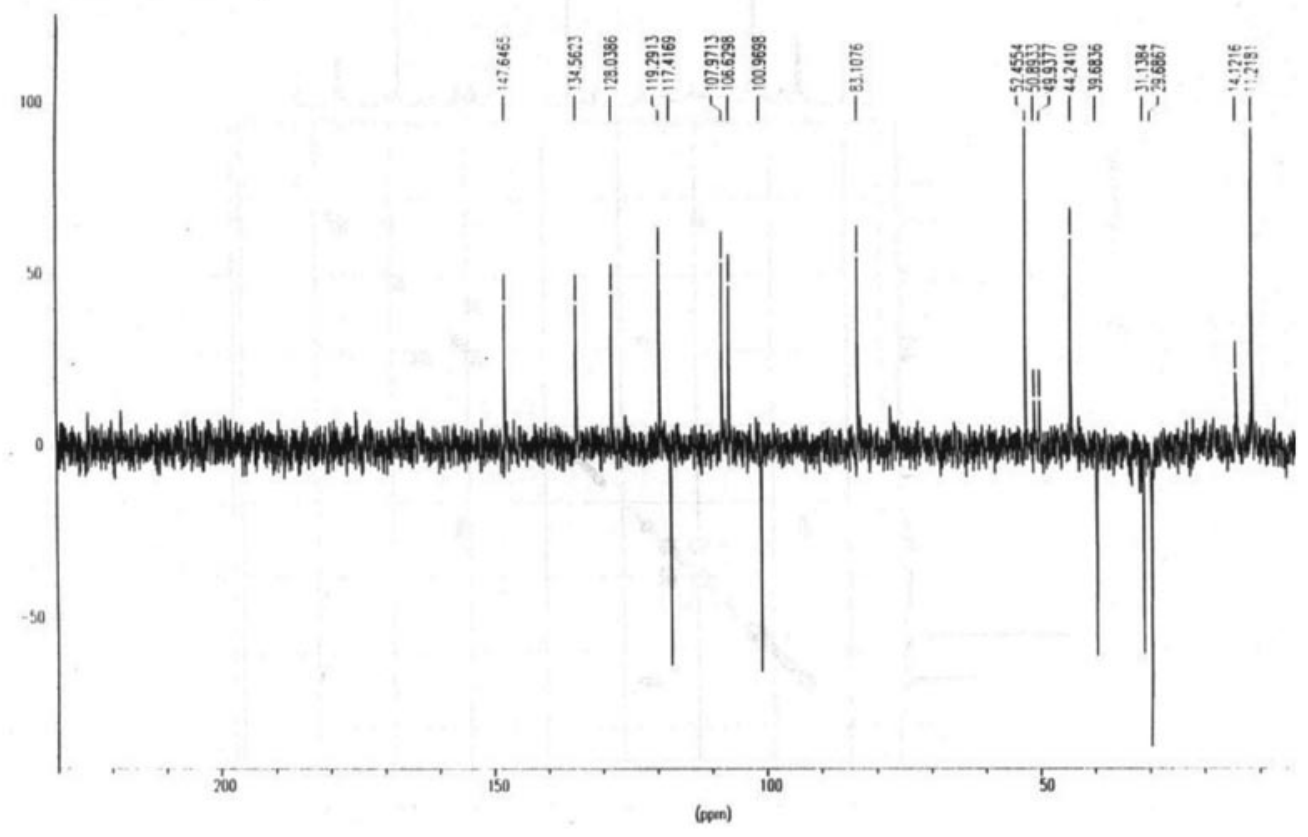

Figure S20. DEPT $135{ }^{13} \mathrm{C}$ NMR spectrum of $2 \mathbf{c}\left(50 \mathrm{MHz}, \mathrm{CDCl}_{3}\right)$.

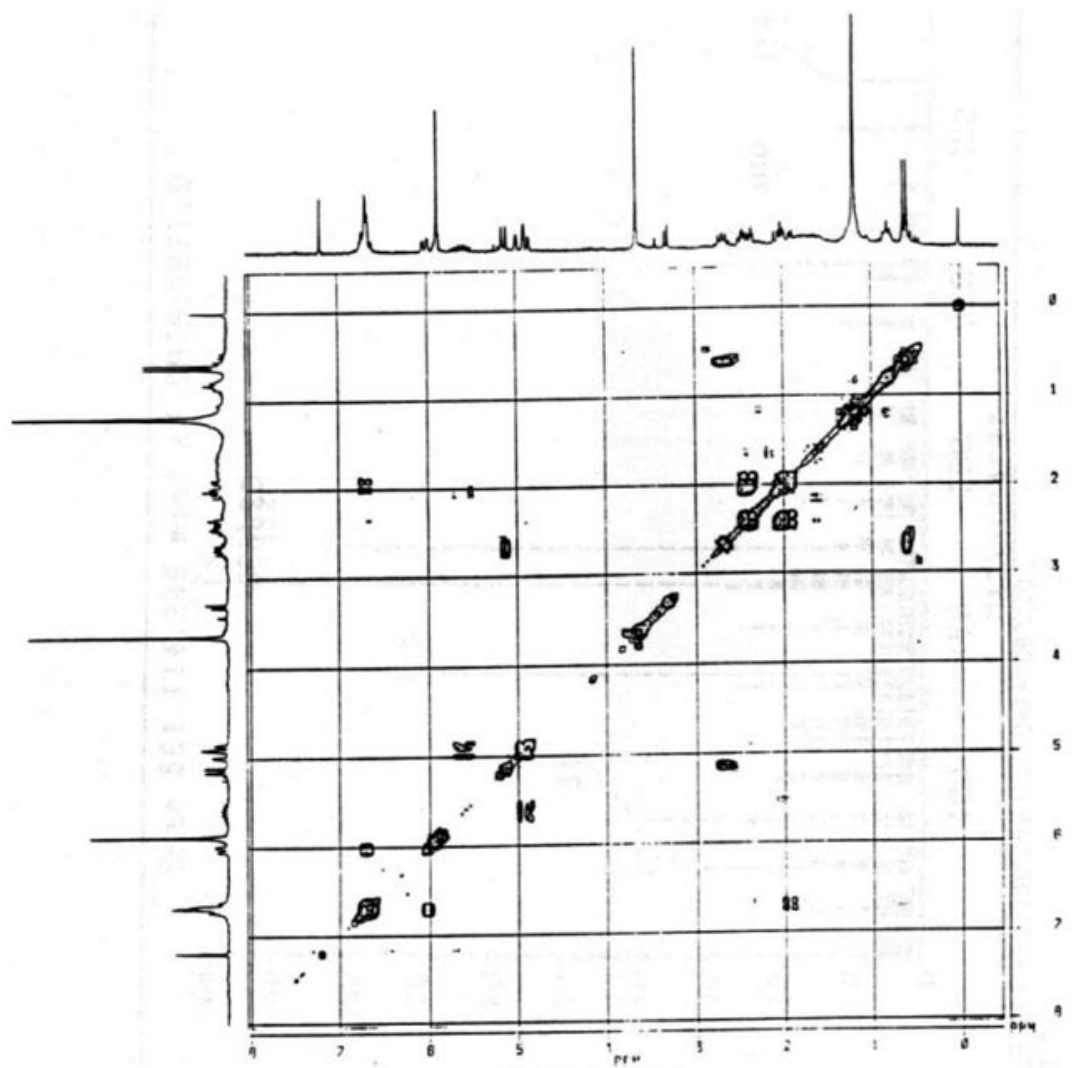

Figure S21. 2D COSY ${ }^{13} \mathrm{C}$ NMR spectrum of $\mathbf{2 c}\left(200 \mathrm{MHz}, \mathrm{CDCl}_{3}\right)$. 


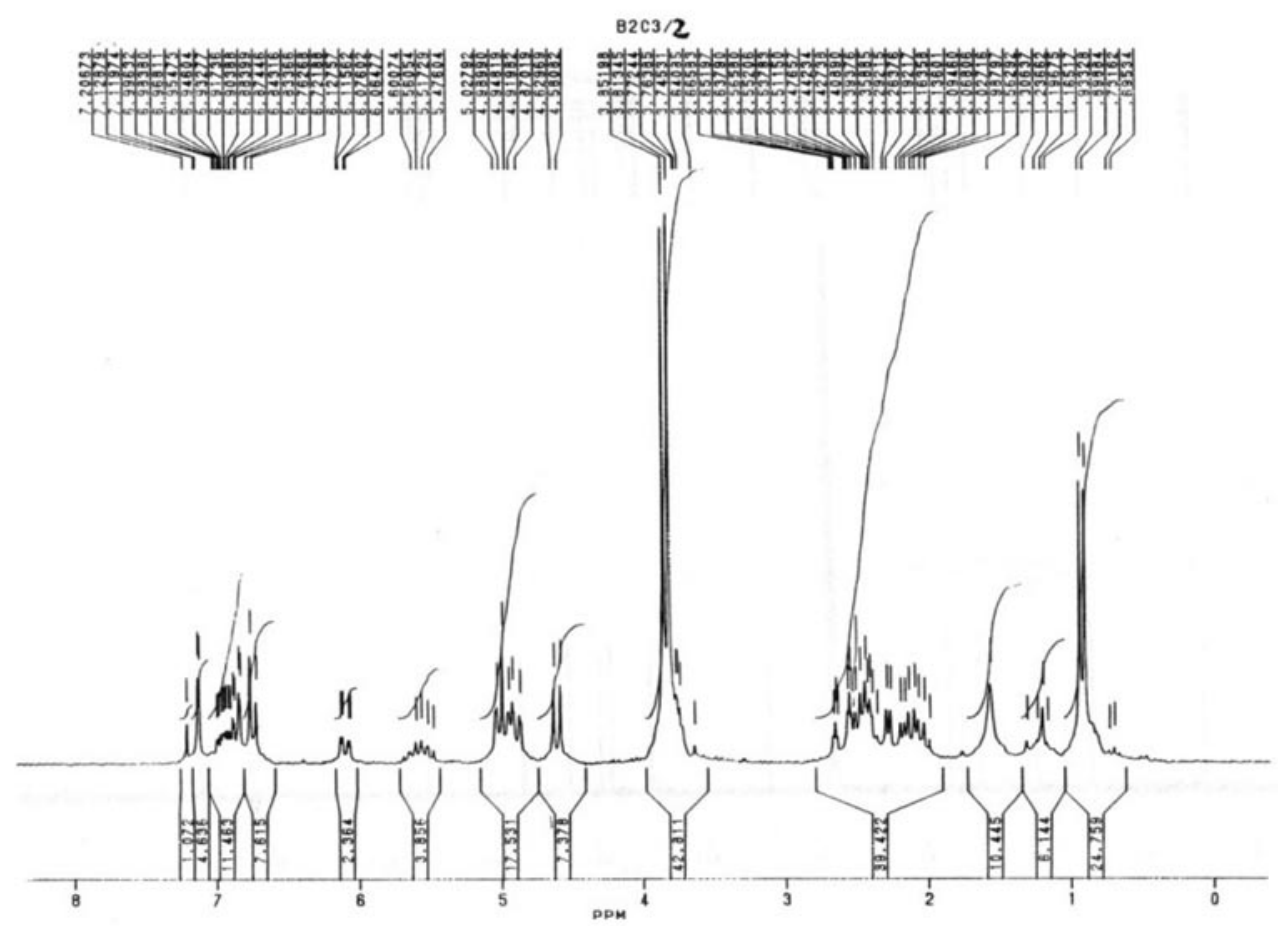

Figure S22. ${ }^{1} \mathrm{H}$ NMR spectrum of $\mathbf{2 d}\left(200 \mathrm{MHz}, \mathrm{CDCl}_{3}\right)$.
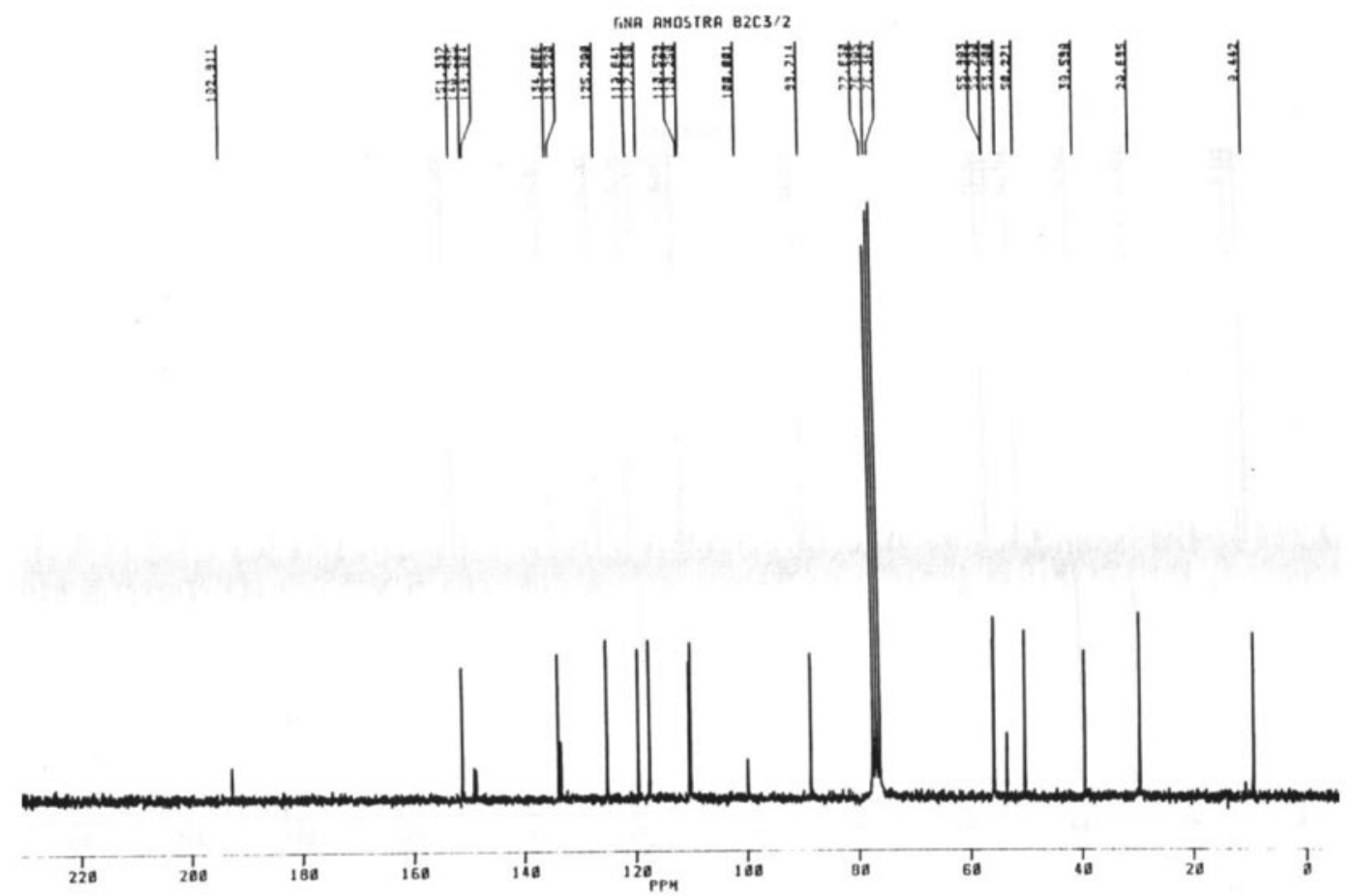

Figure S23. ${ }^{13} \mathrm{C}$ NMR spectrum of $\mathbf{2 d}\left(50 \mathrm{MHz}, \mathrm{CDCl}_{3}\right)$. 


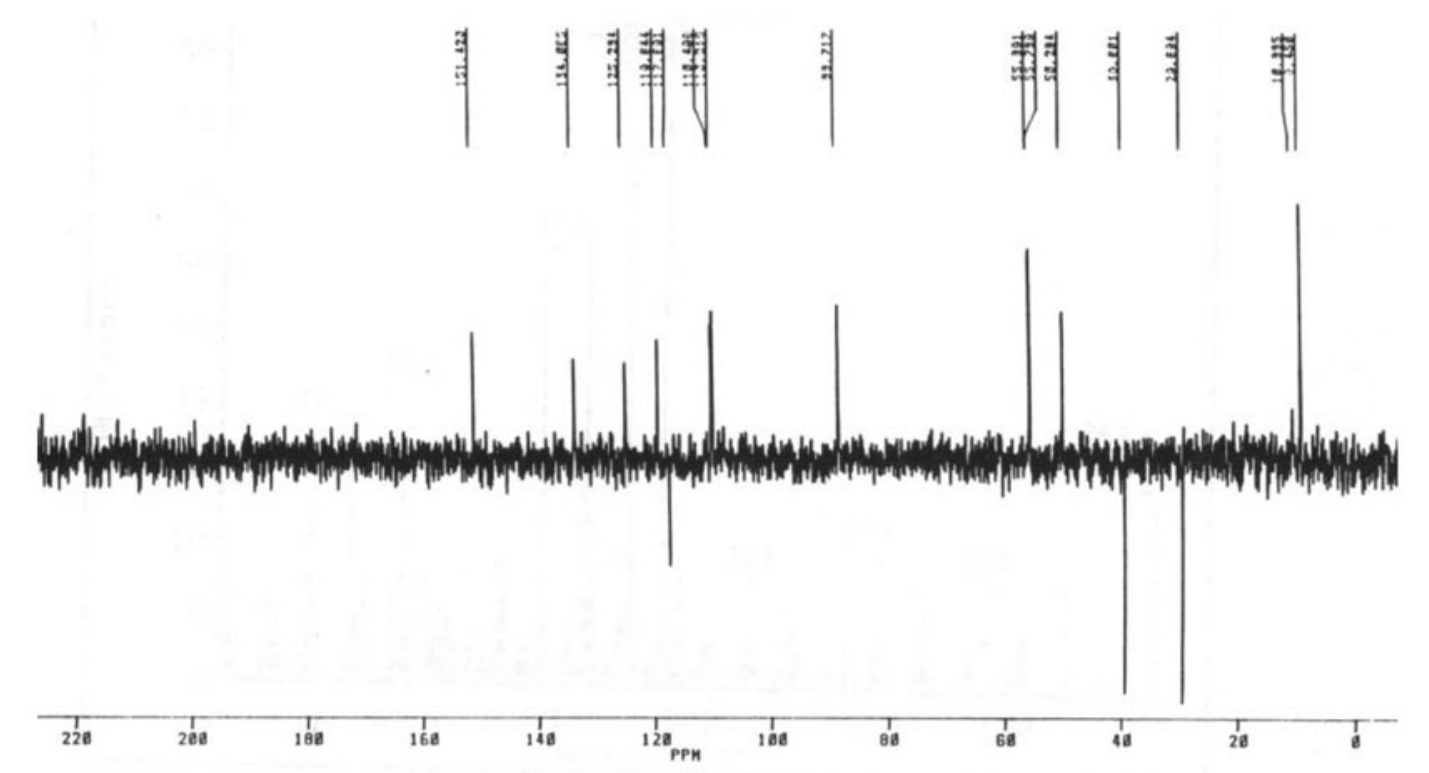

Figure S24. DEPT $135{ }^{13} \mathrm{C}$ NMR spectrum of $2 d\left(50 \mathrm{MHz}, \mathrm{CDCl}_{3}\right)$.

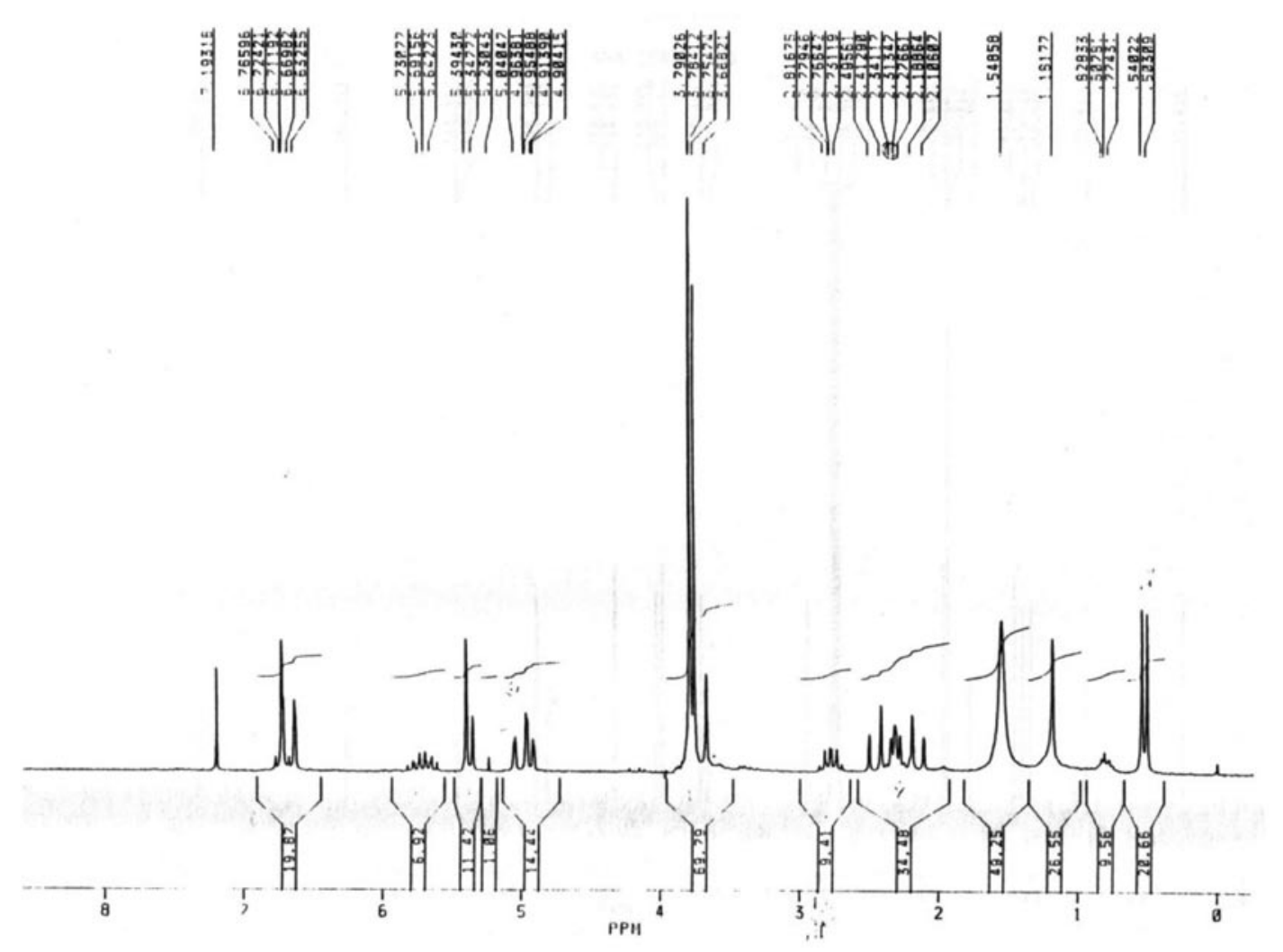

Figure S25. ' $\mathrm{H}$ NMR spectrum of $\mathbf{2 f}\left(200 \mathrm{MHz}, \mathrm{CDCl}_{3}\right)$. 


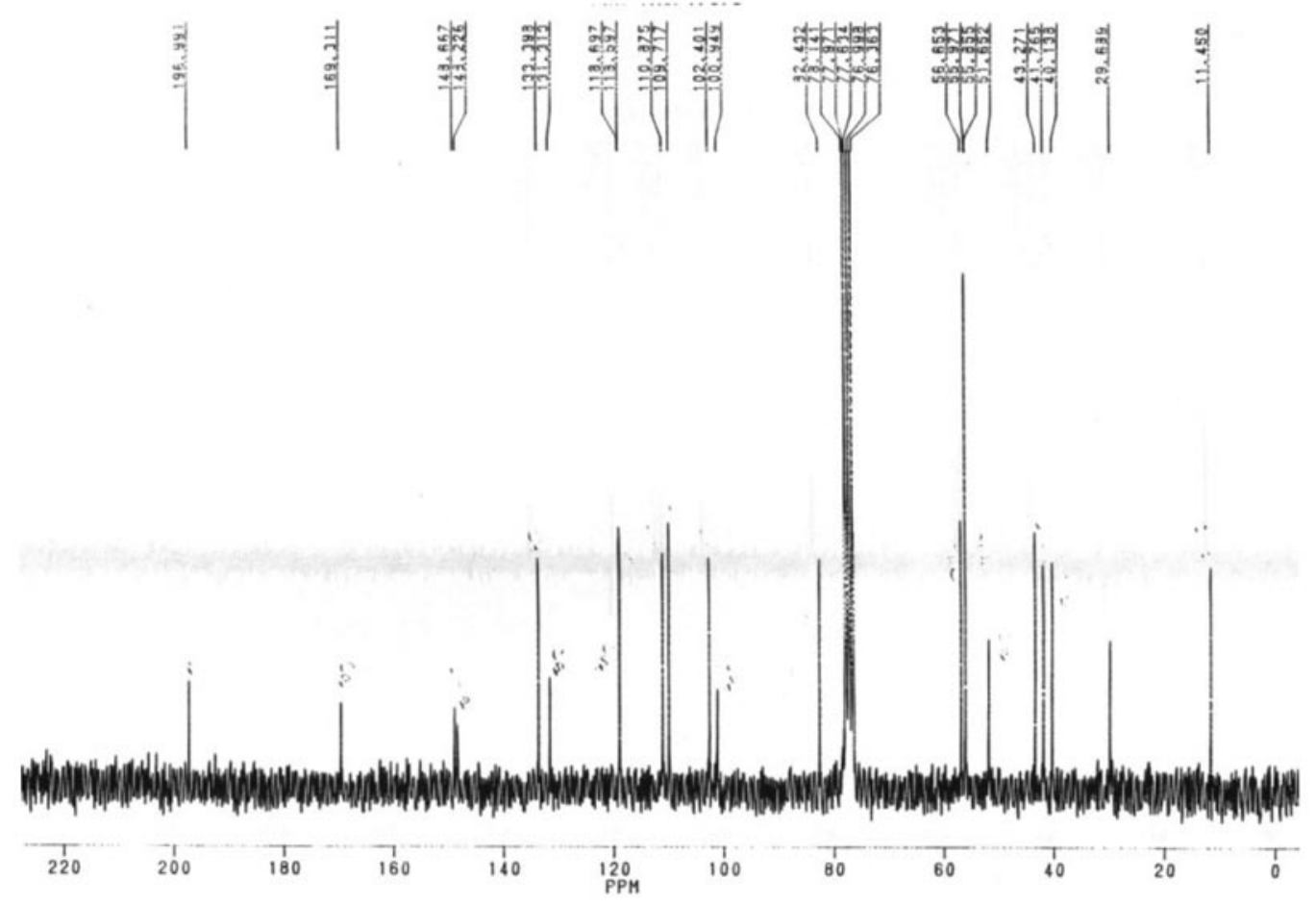

Figure S26. ${ }^{13} \mathrm{C}$ NMR spectrum of $\mathbf{2 f}\left(50 \mathrm{MHz}, \mathrm{CDCl}_{3}\right)$.

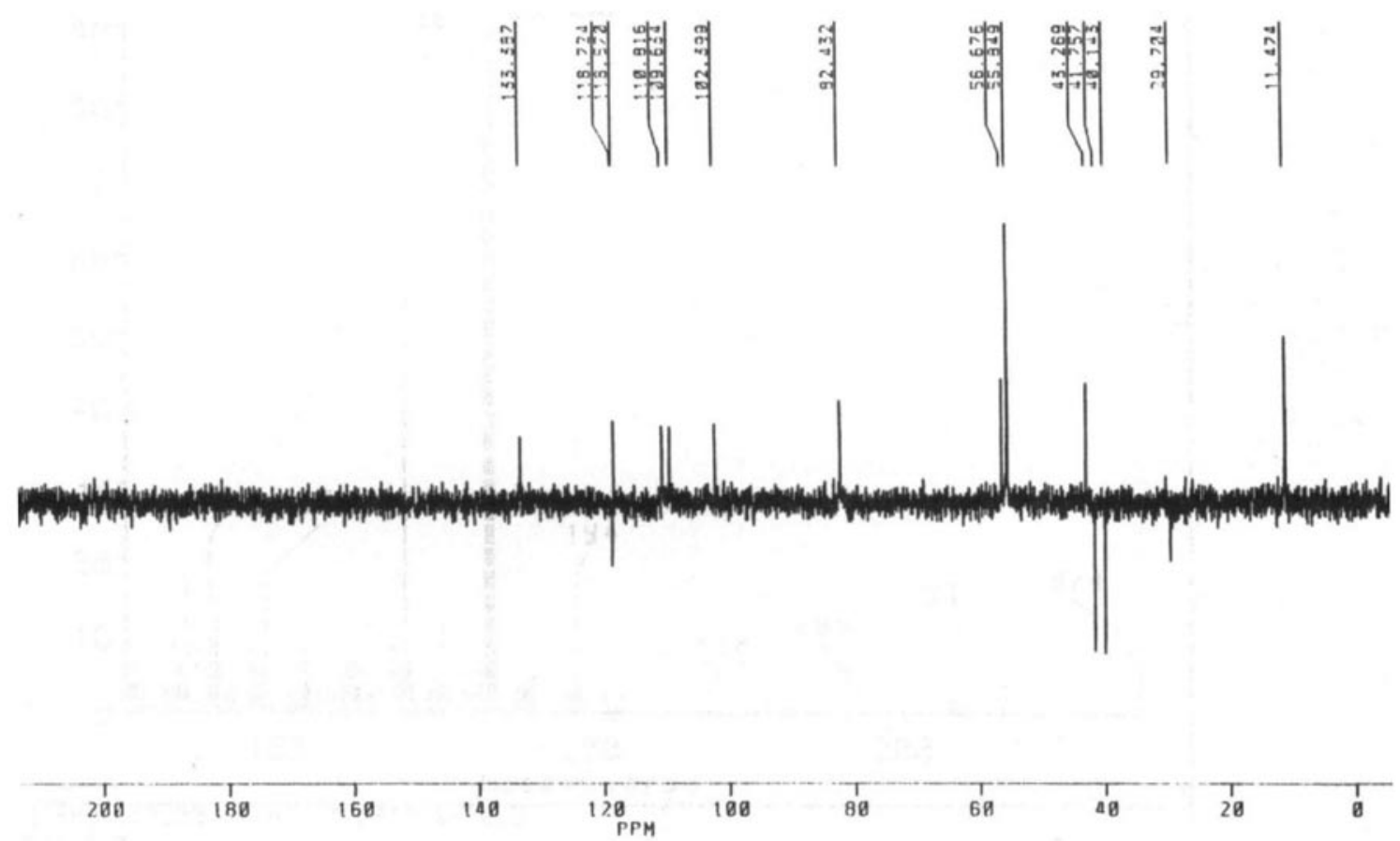

Figure S27. DEPT $135{ }^{13} \mathrm{C}$ NMR spectrum of $\mathbf{2 f}\left(50 \mathrm{MHz}, \mathrm{CDCl}_{3}\right)$. 


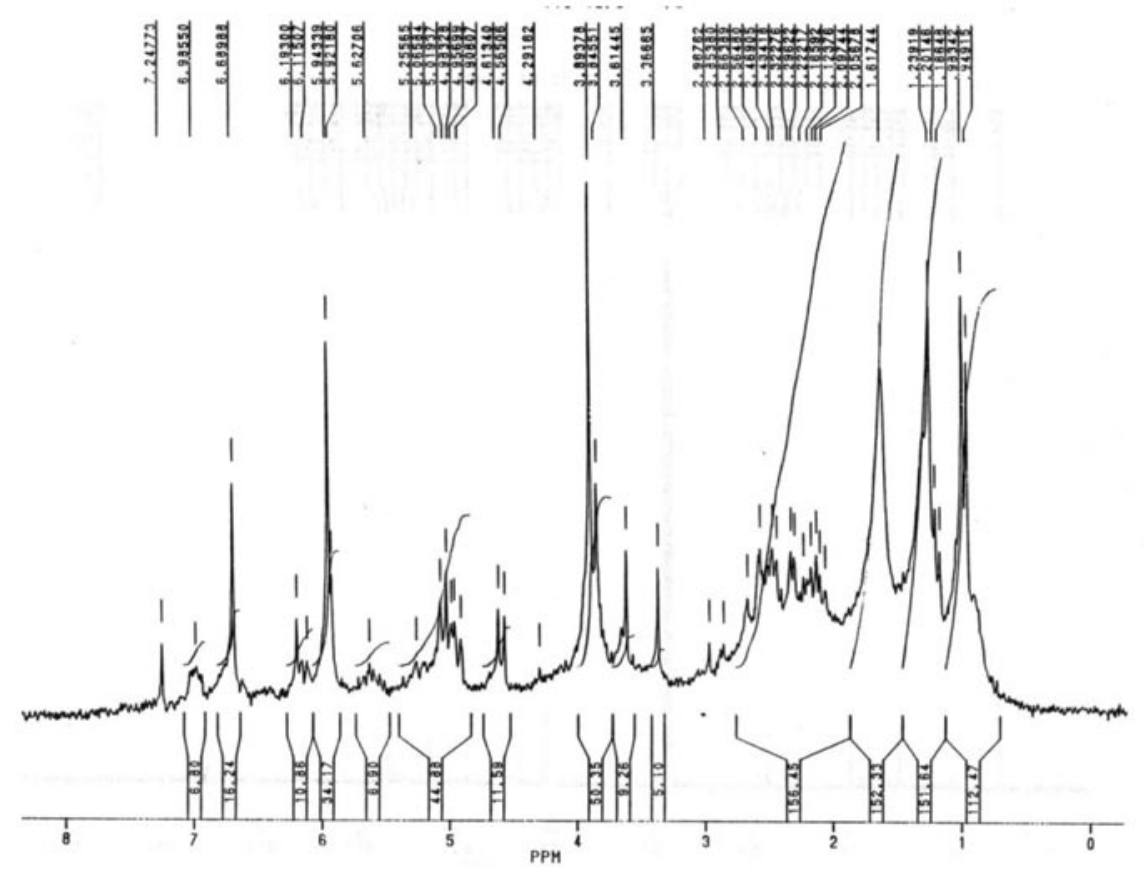

Figure S28. ${ }^{1} \mathrm{H}$ NMR spectrum of $\mathbf{2 g}\left(200 \mathrm{MHz}, \mathrm{CDCl}_{3}\right)$.
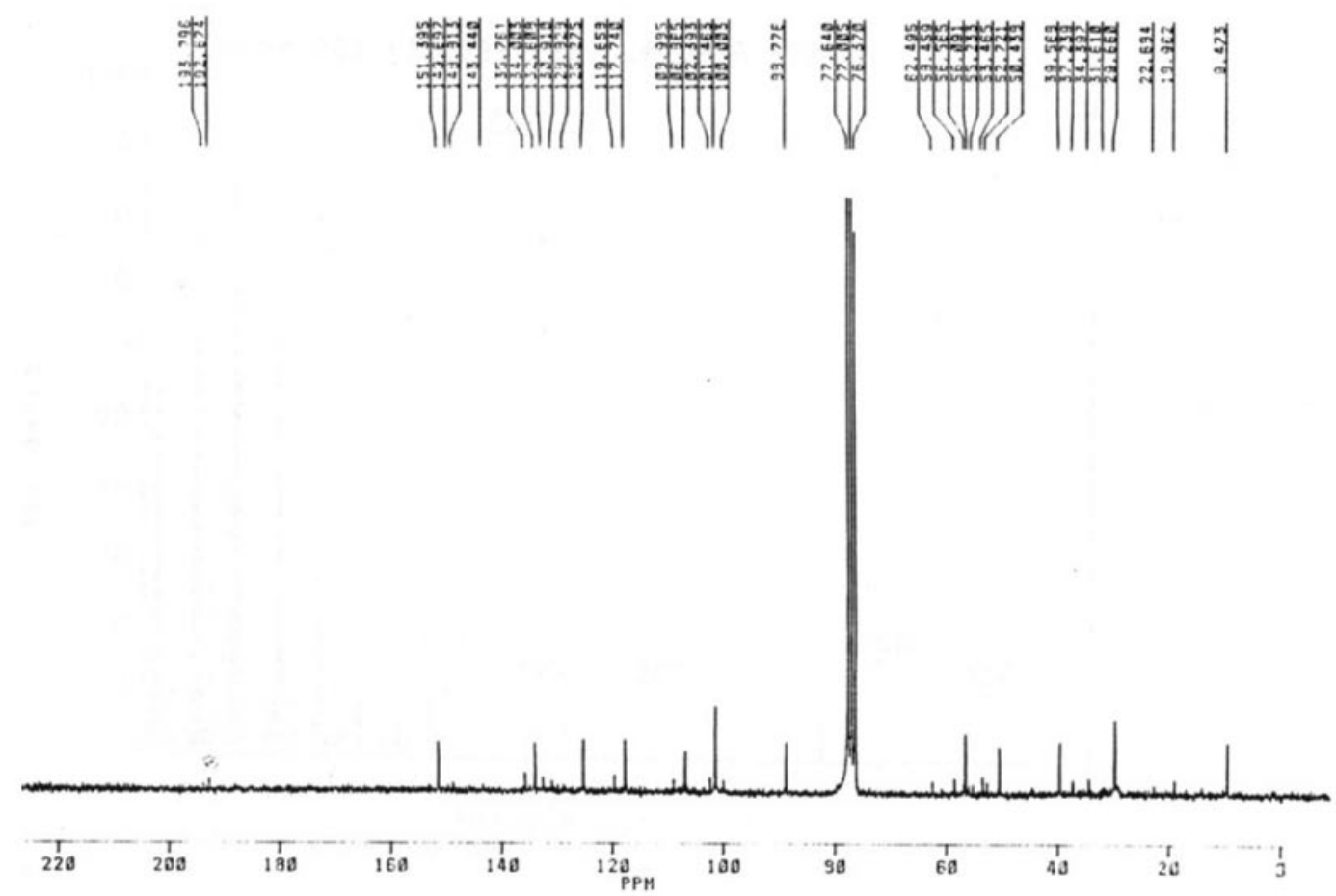

Figure S29. ${ }^{13} \mathrm{C}$ NMR spectrum of $\mathbf{2 g}\left(50 \mathrm{MHz}, \mathrm{CDCl}_{3}\right)$. 


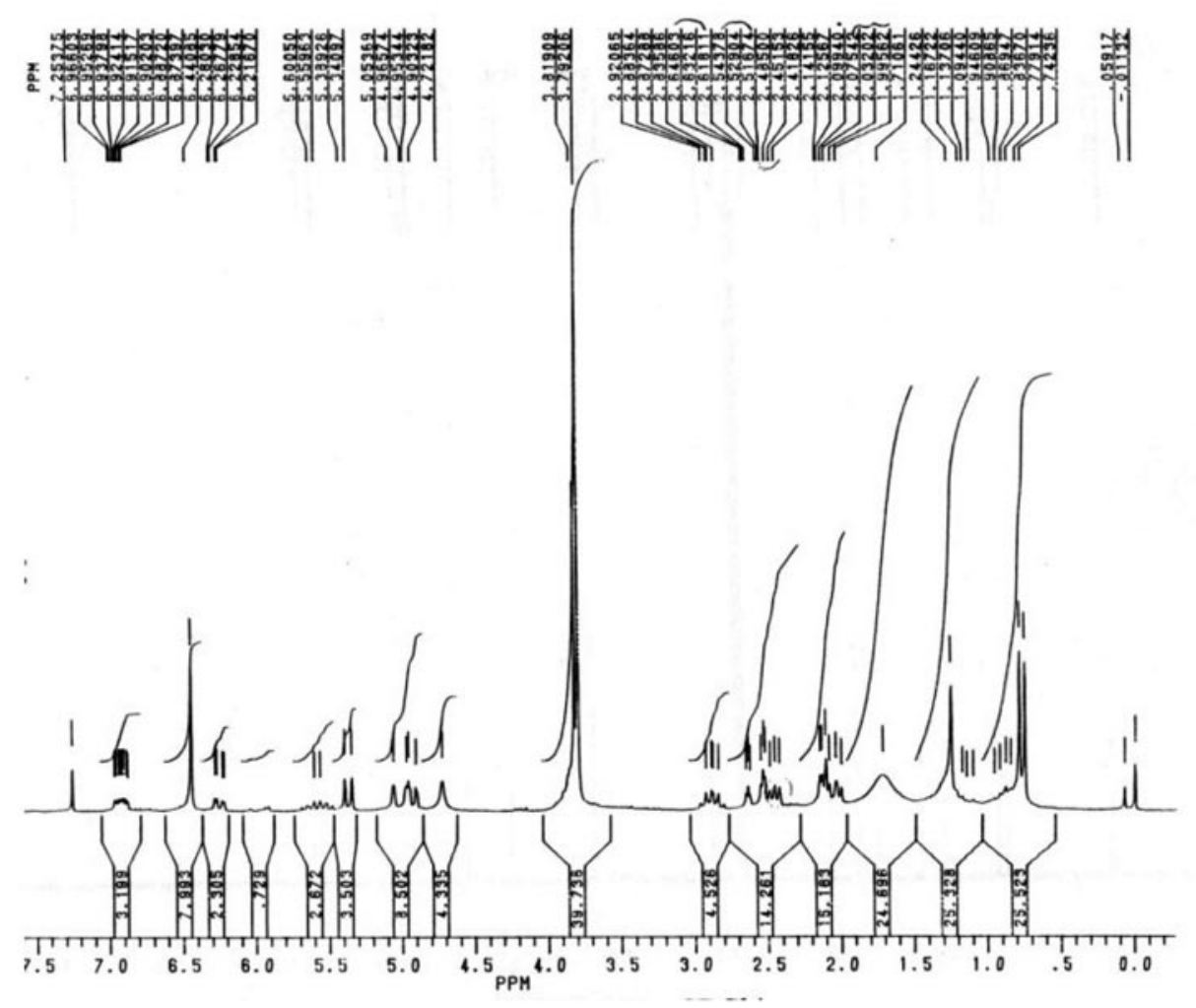

Figure S30. ${ }^{1} \mathrm{H}$ NMR spectrum of $\mathbf{2 h}\left(200 \mathrm{MHz}, \mathrm{CDCl}_{3}\right)$.

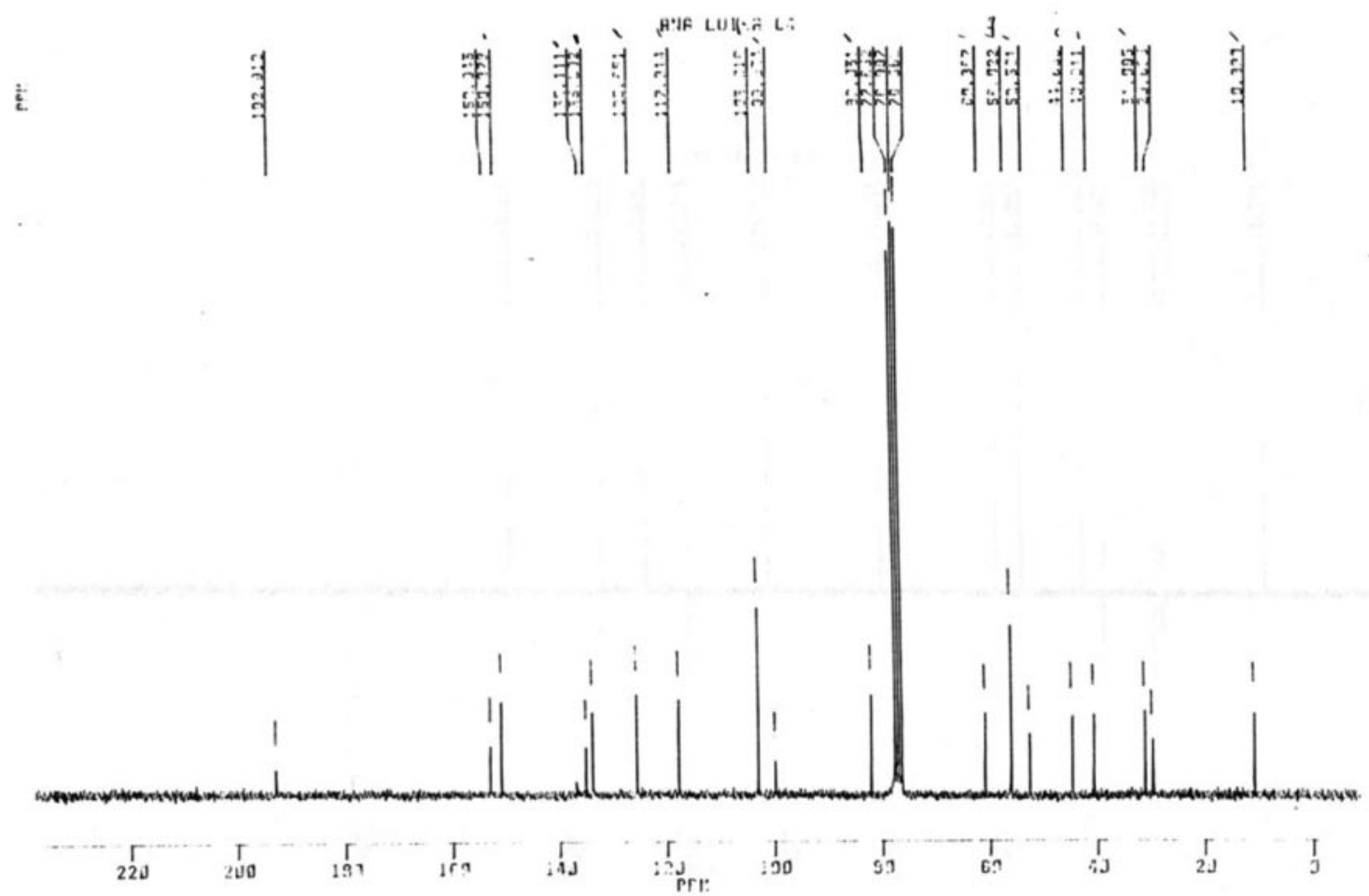

Figure S31. ${ }^{13} \mathrm{C}$ NMR spectrum of $\mathbf{2 h}\left(50 \mathrm{MHz}, \mathrm{CDCl}_{3}\right)$. 


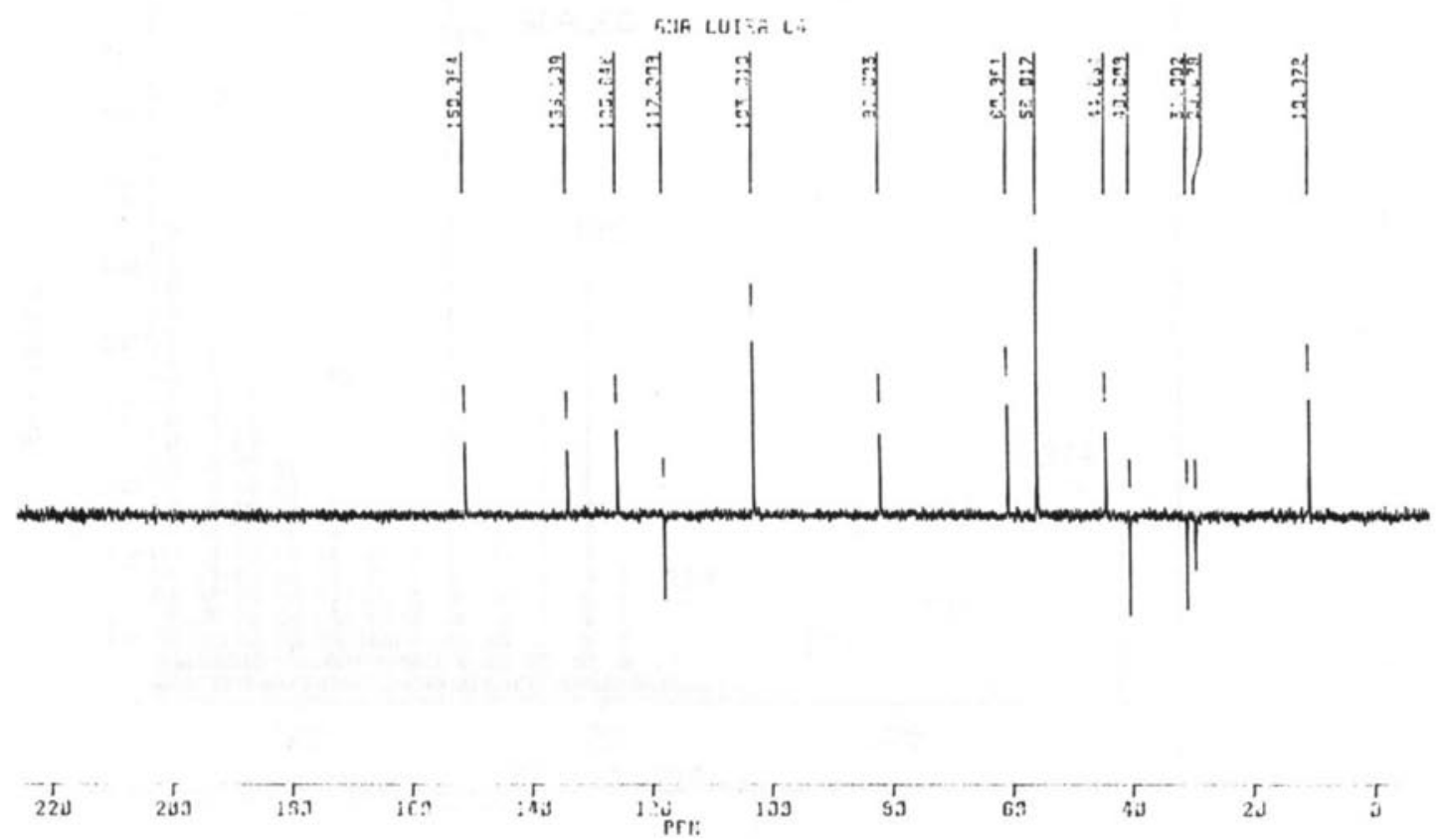

Figure S32. DEPT $135{ }^{13} \mathrm{C}$ NMR spectrum of $\mathbf{2 h}\left(50 \mathrm{MHz}, \mathrm{CDCl}_{3}\right)$.

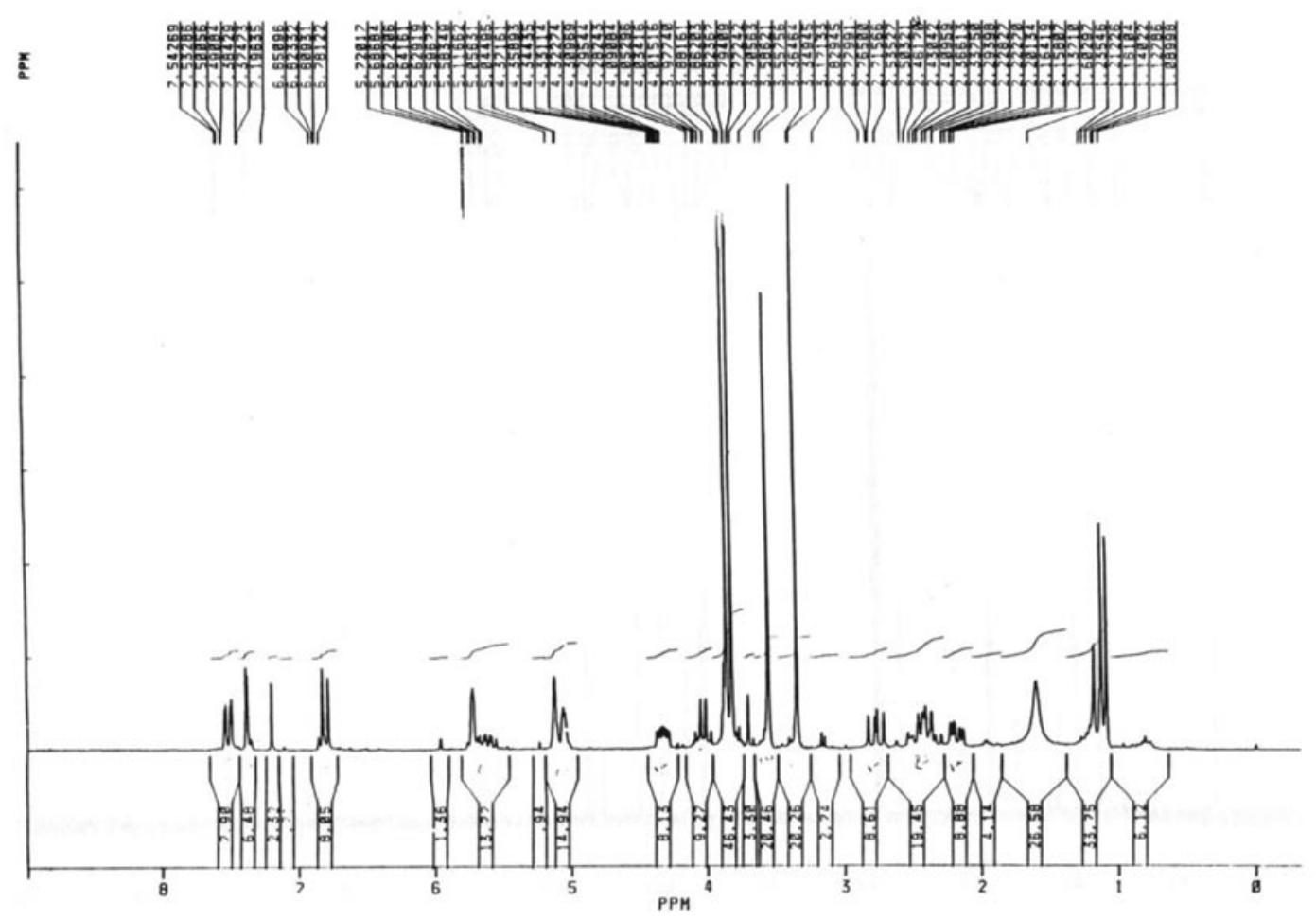

Figure S33. ${ }^{1} \mathrm{H}$ NMR spectrum of $\mathbf{3 b}\left(200 \mathrm{MHz}, \mathrm{CDCl}_{3}\right)$. 


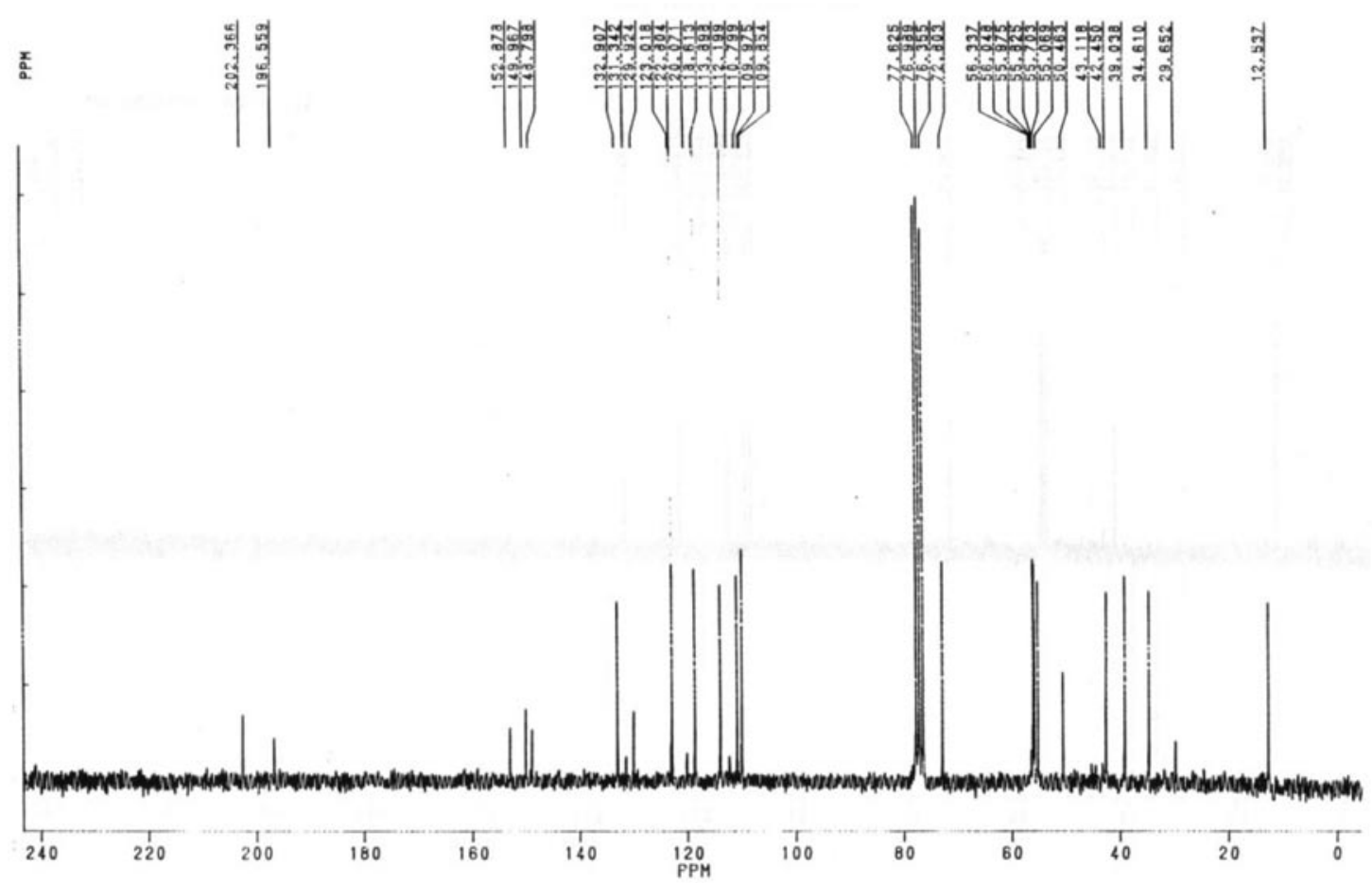

Figure S34. ${ }^{13} \mathrm{C}$ NMR spectrum of $\mathbf{3 b}\left(50 \mathrm{MHz}, \mathrm{CDCl}_{3}\right)$.
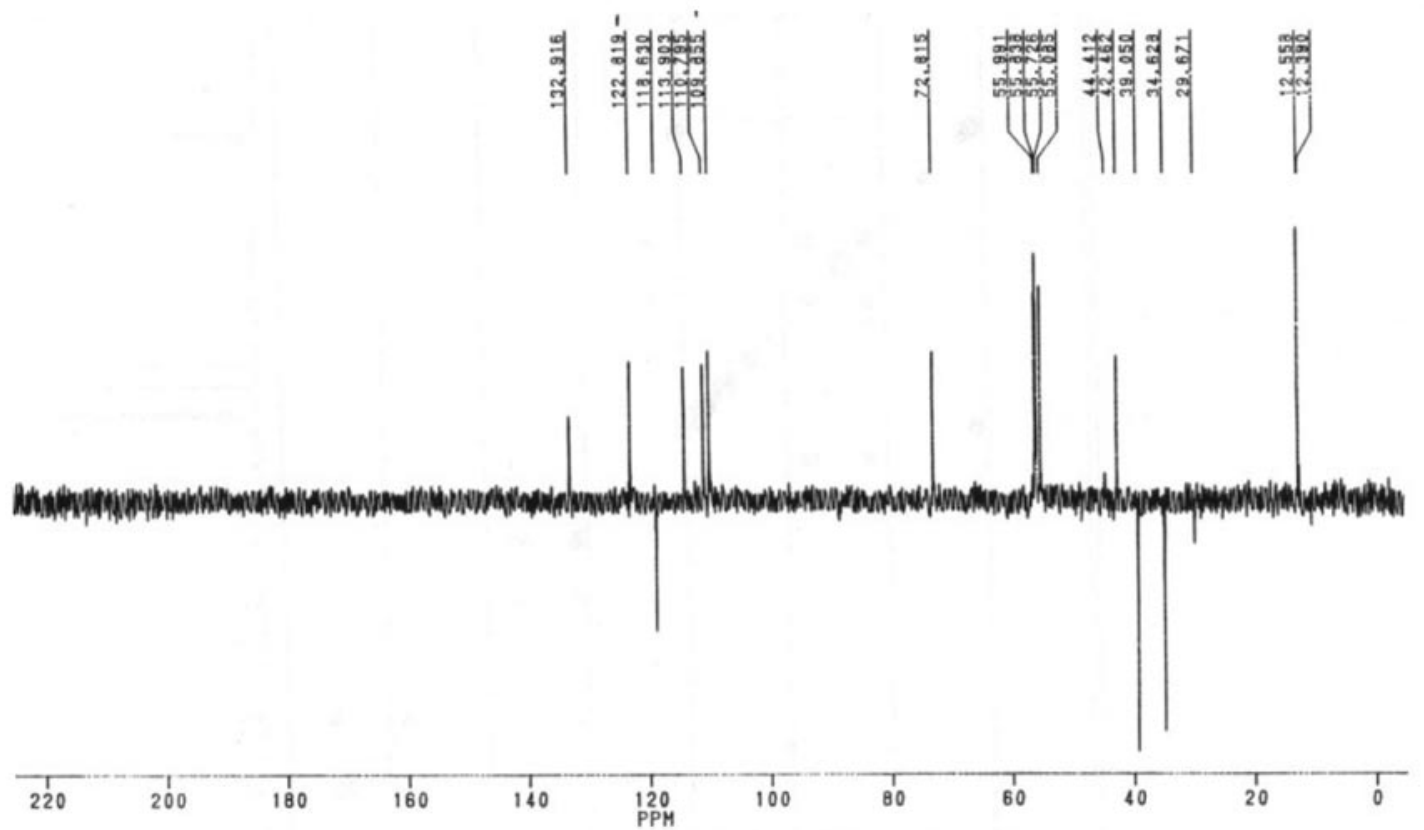

Figure S35. DEPT $135{ }^{13} \mathrm{C}$ NMR spectrum of $\mathbf{3 b}\left(50 \mathrm{MHz}, \mathrm{CDCl}_{3}\right)$. 


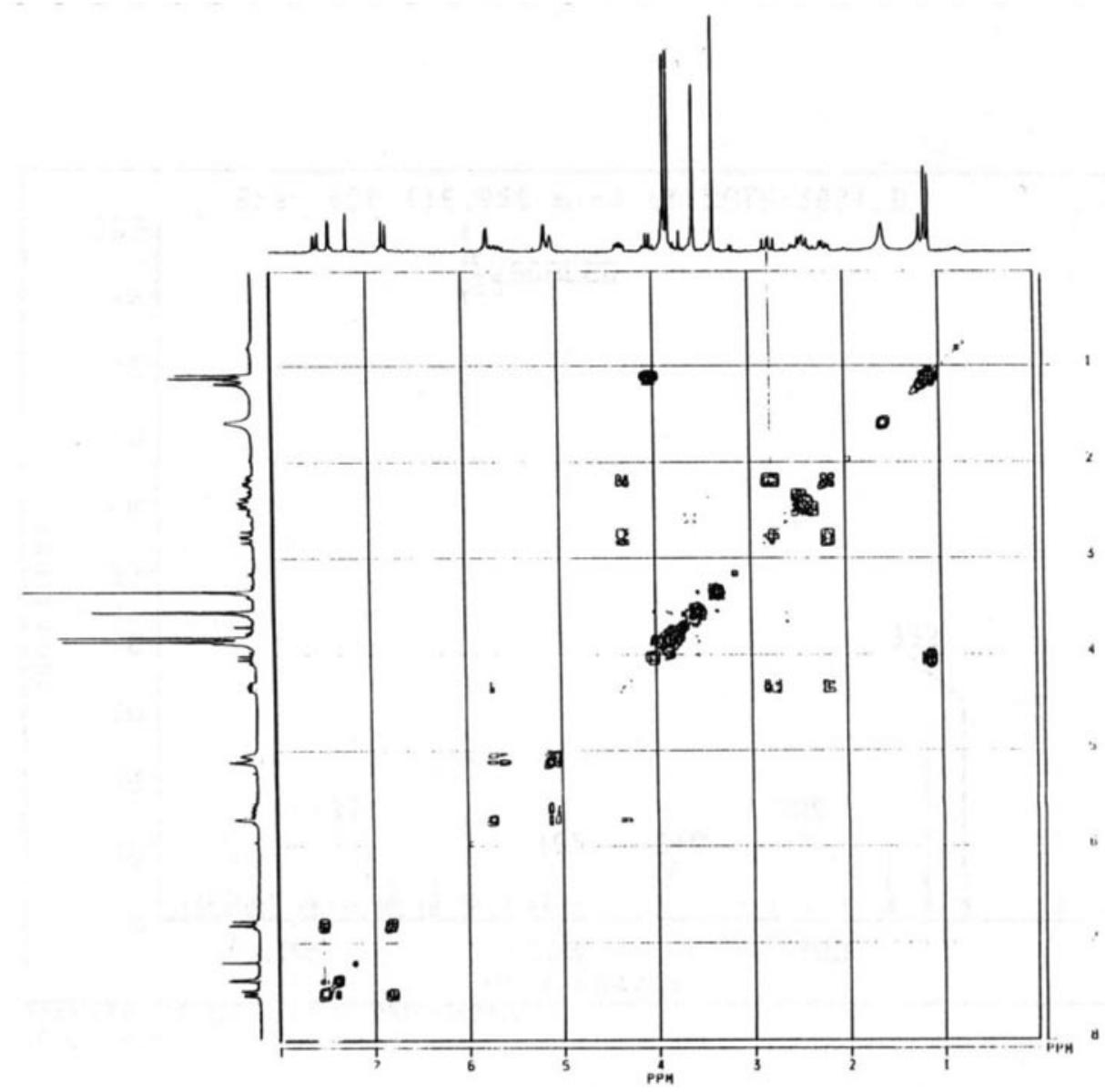

Figure S36. COSY ${ }^{13} \mathrm{C}$ NMR spectrum of $\mathbf{3 b}\left(50 \mathrm{MHz}, \mathrm{CDCl}_{3}\right)$.

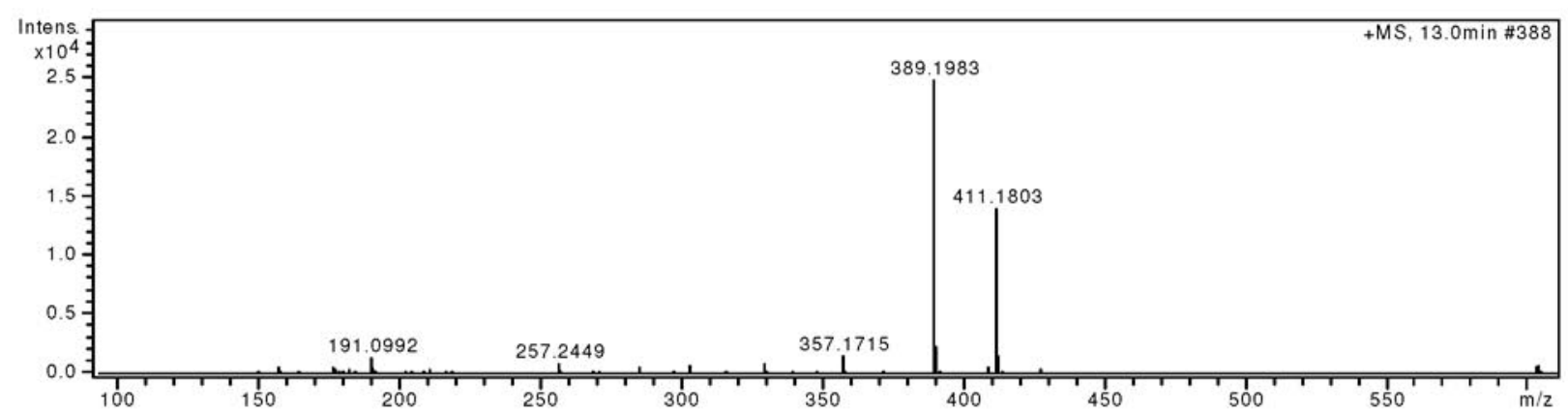

Figure S37. HRESIMS spectrum of $\mathbf{3 b}$. 


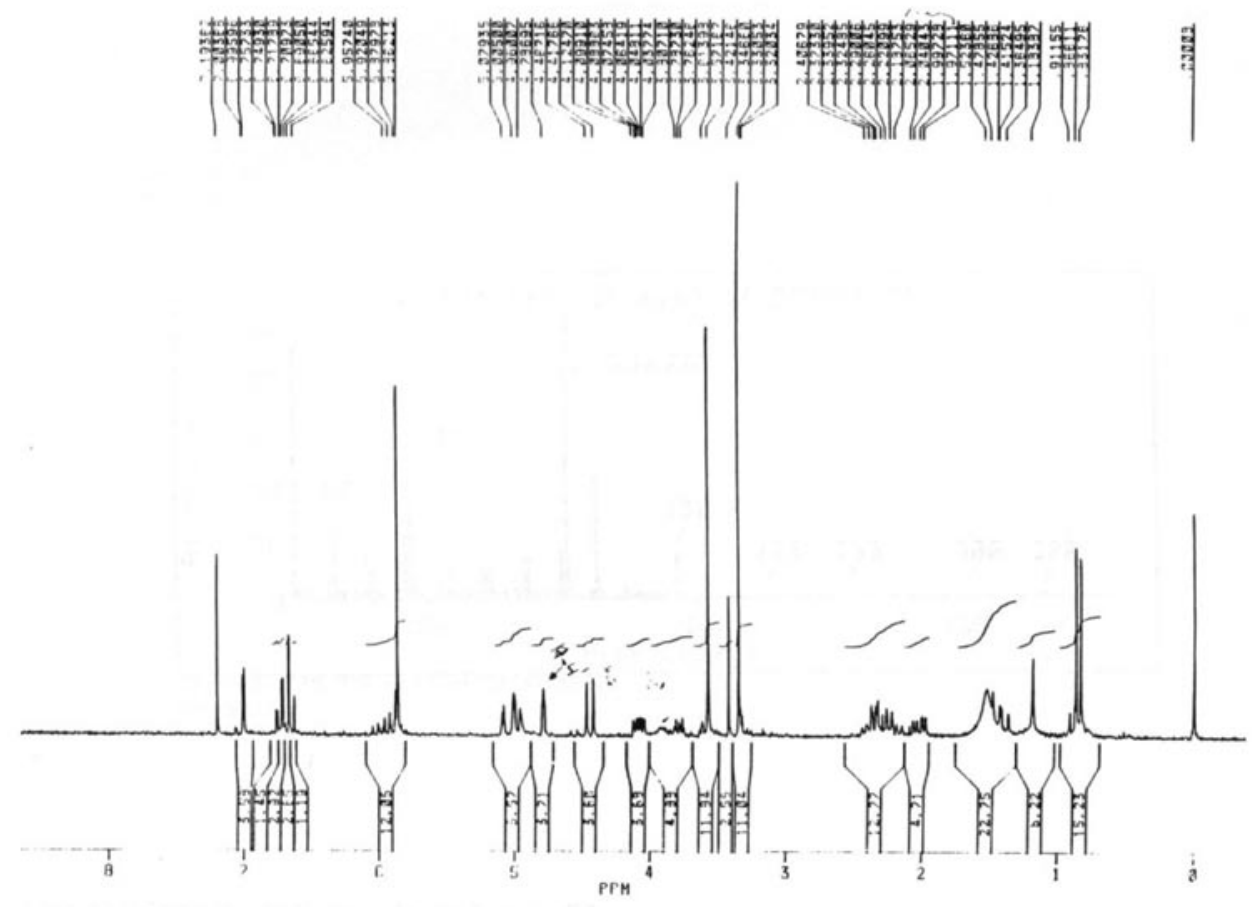

Figure S38. ${ }^{1} \mathrm{H}$ NMR spectrum of $4 \mathbf{a}\left(200 \mathrm{MHz}, \mathrm{CDCl}_{3}\right)$.

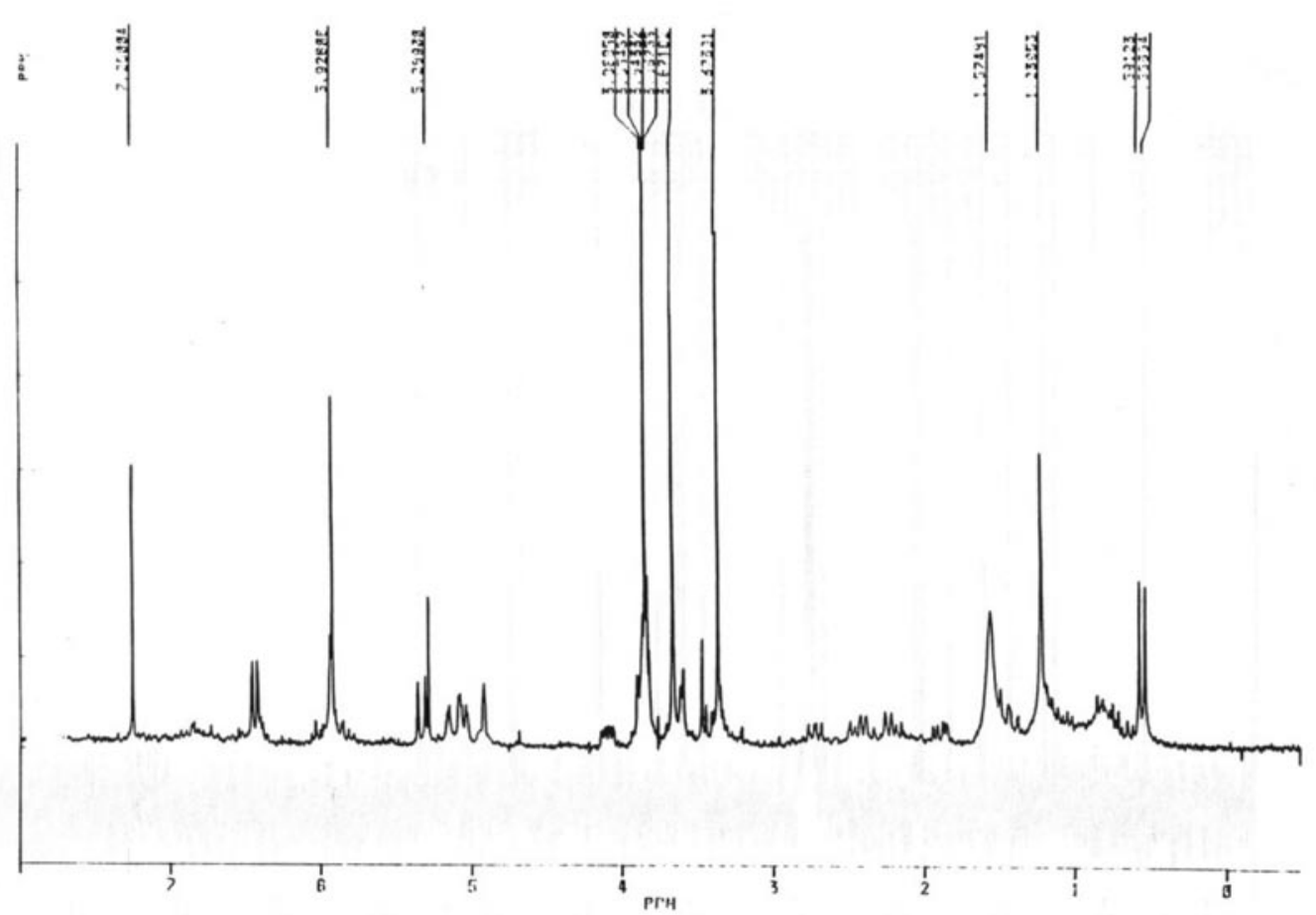

Figure S39. ${ }^{1} \mathrm{H}$ NMR spectrum of $\mathbf{4 b}\left(200 \mathrm{MHz}, \mathrm{CDCl}_{3}\right)$. 


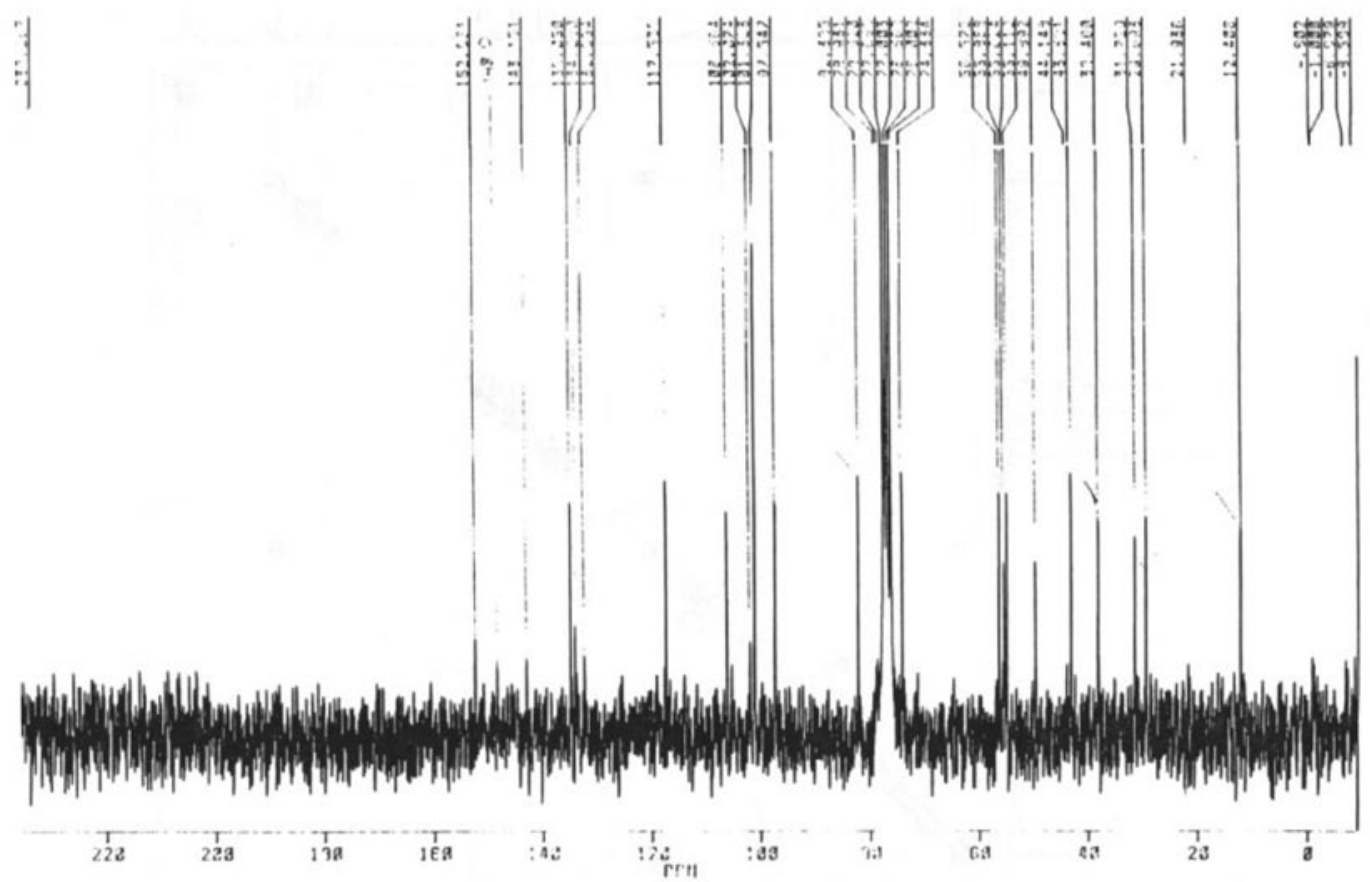

Figure S40. ${ }^{13} \mathrm{C}$ NMR spectrum of $\mathbf{4 b}\left(50 \mathrm{MHz}, \mathrm{CDCl}_{3}\right)$.
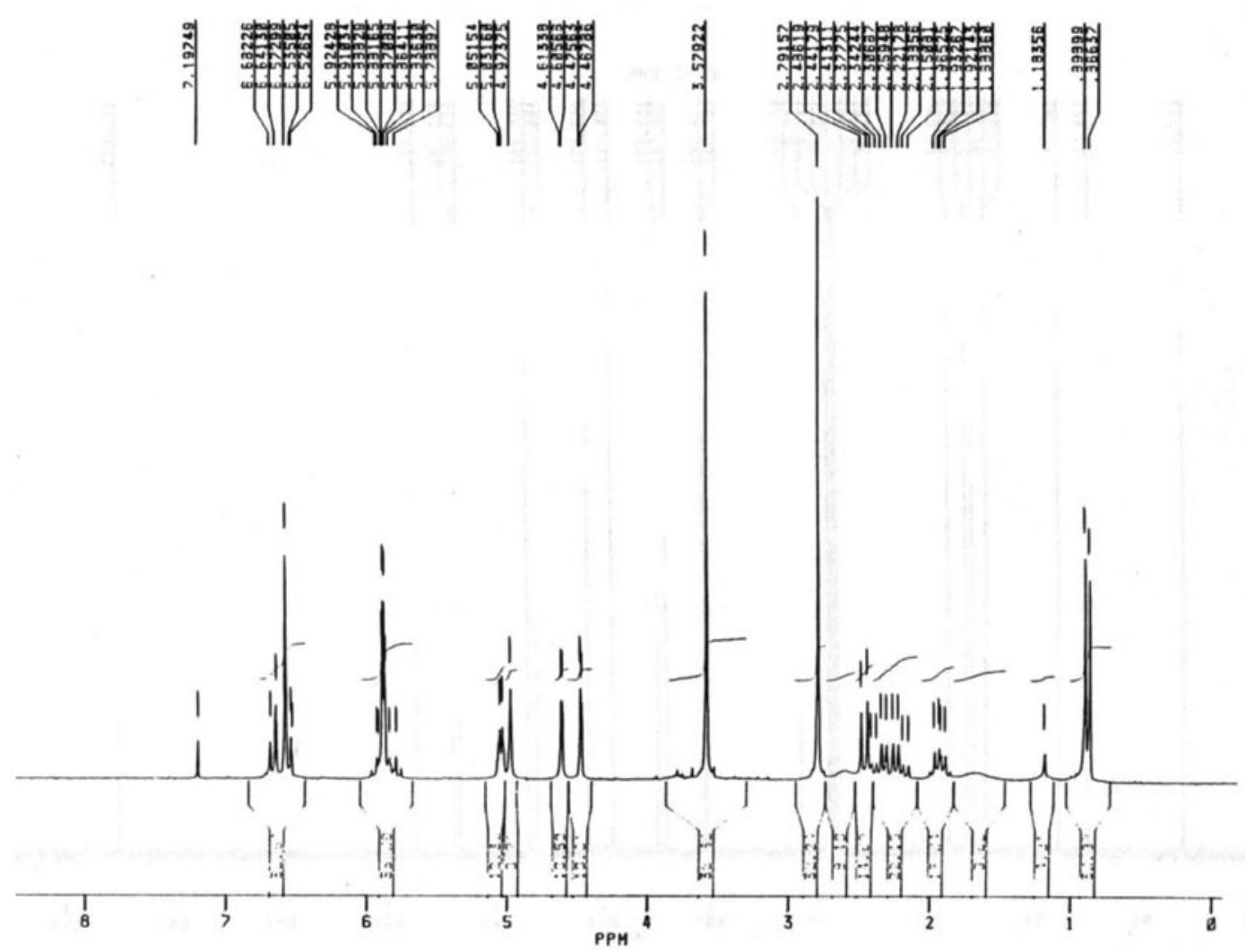

Figure S41. ${ }^{1} \mathrm{H} \mathrm{NMR}$ spectrum of $\mathbf{5 a}\left(200 \mathrm{MHz}, \mathrm{CDCl}_{3}\right)$. 


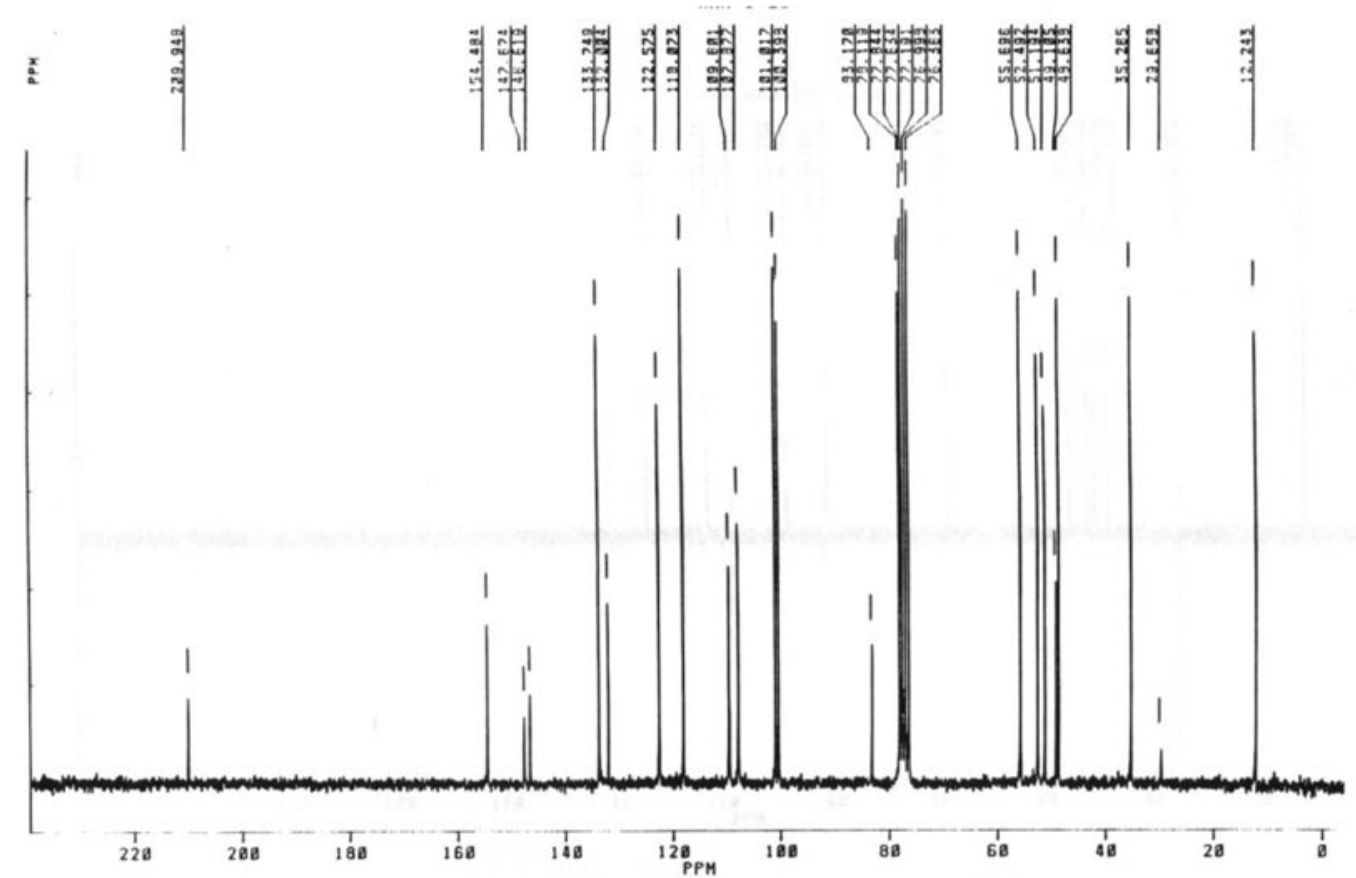

Figure S42. ${ }^{13} \mathrm{C}$ NMR spectrum of $\mathbf{5 a}\left(50 \mathrm{MHz}, \mathrm{CDCl}_{3}\right)$.

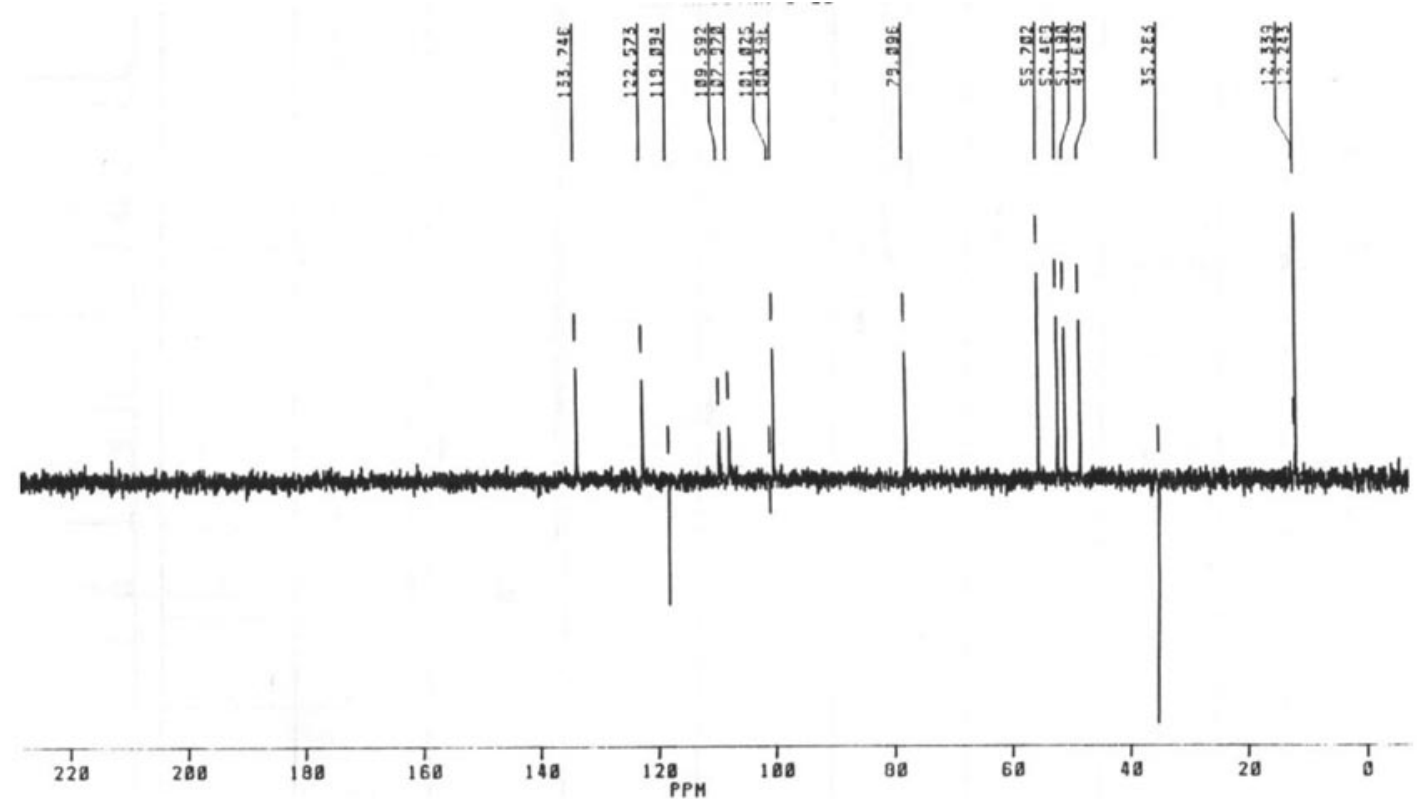

Figure S43. DEPT $135{ }^{13} \mathrm{C}$ NMR spectrum of $\mathbf{5 a}\left(50 \mathrm{MHz}, \mathrm{CDCl}_{3}\right)$. 


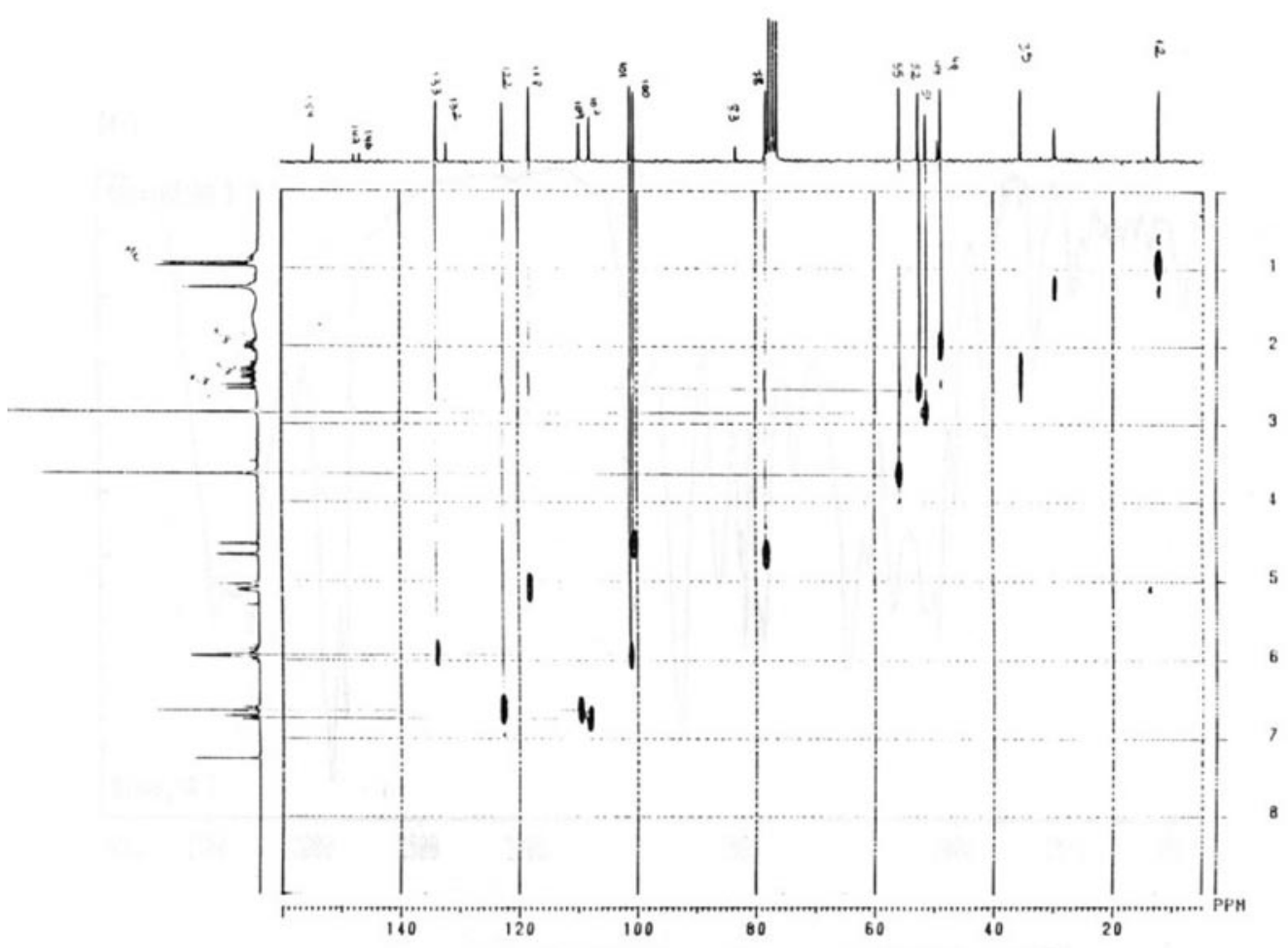

Figure S44. $\mathrm{HETCOR}{ }^{13} \mathrm{C}$ NMR spectrum of $5 \mathbf{a}\left(50 \mathrm{MHz}, \mathrm{CDCl}_{3}\right)$.

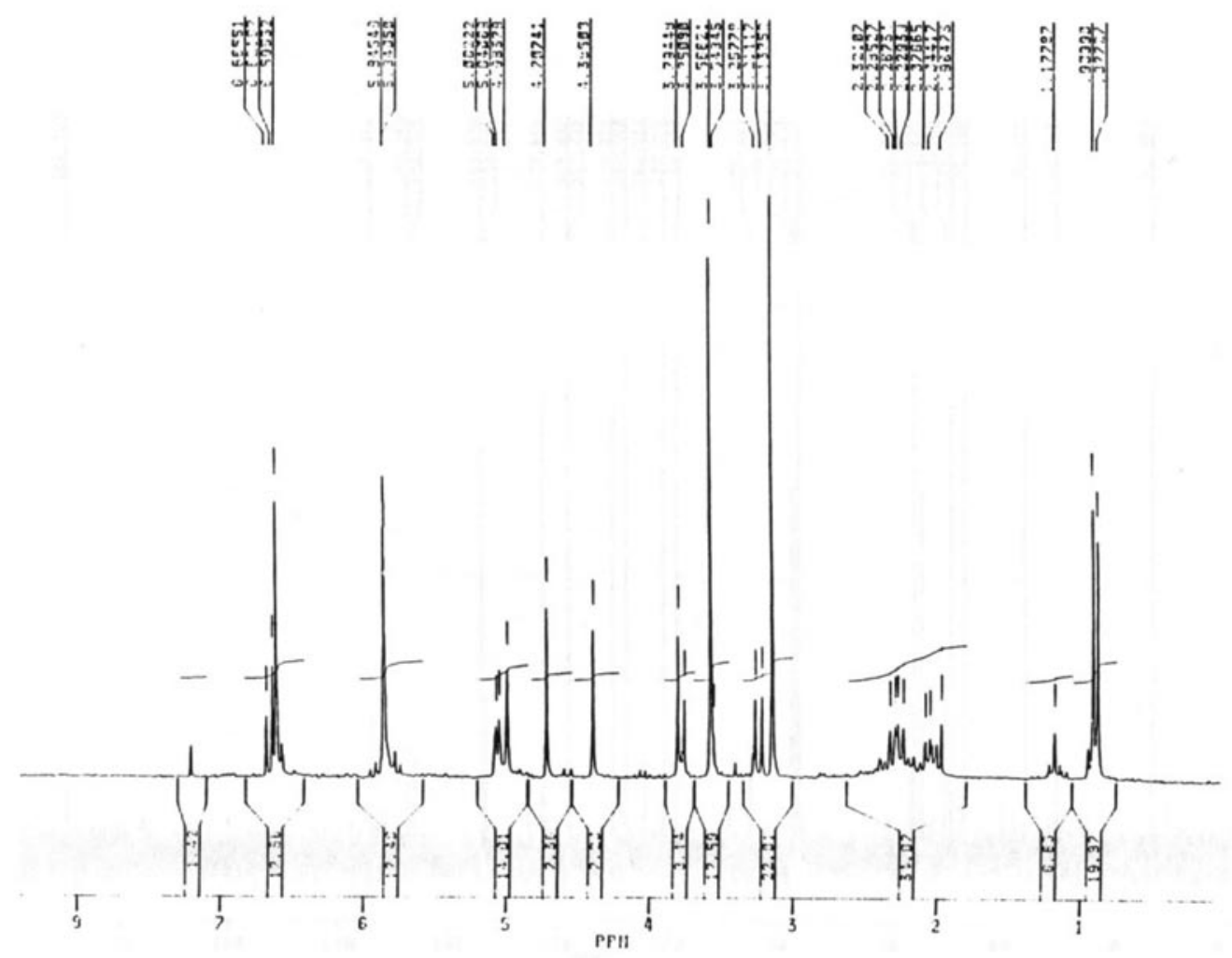

Figure S45. ${ }^{1} \mathrm{H}$ NMR spectrum of $\mathbf{5 b}\left(200 \mathrm{MHz}, \mathrm{CDCl}_{3}\right)$. 

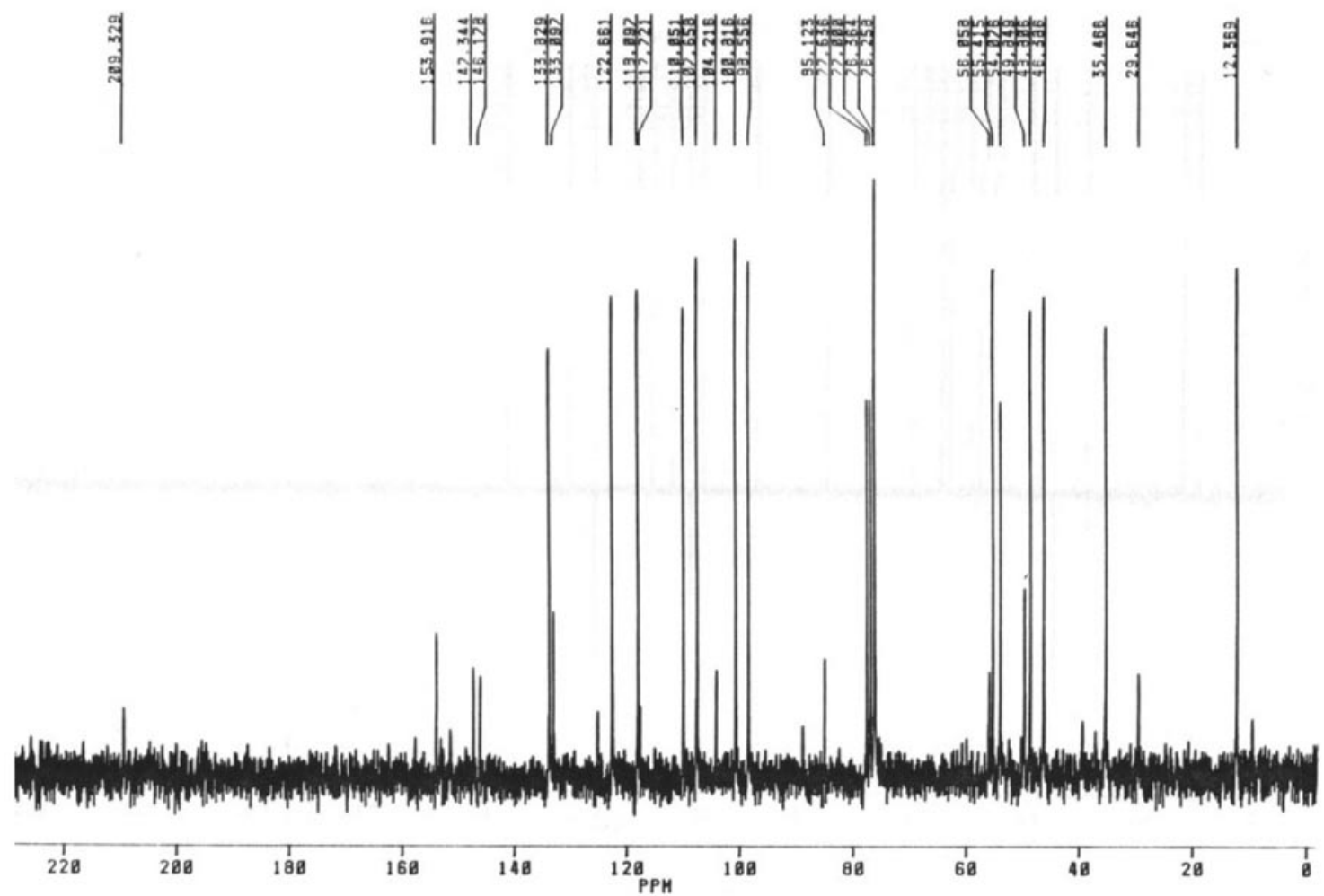

Figure S46. ${ }^{13} \mathrm{C}$ NMR spectrum of $\mathbf{5 b}\left(50 \mathrm{MHz}, \mathrm{CDCl}_{3}\right)$.
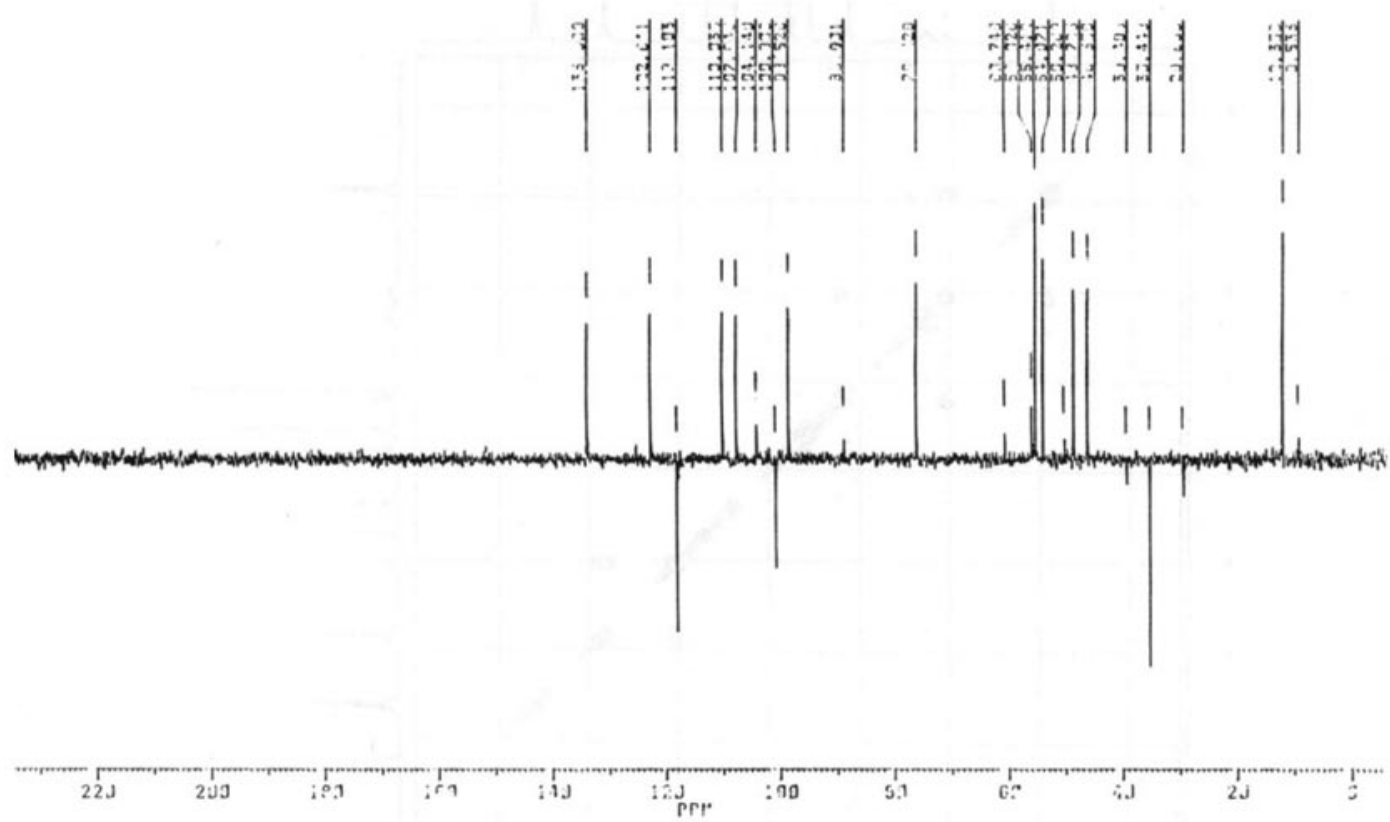

Figure S47. DEPT $135{ }^{13} \mathrm{C}$ NMR spectrum of $\mathbf{5 b}\left(50 \mathrm{MHz}, \mathrm{CDCl}_{3}\right)$. 


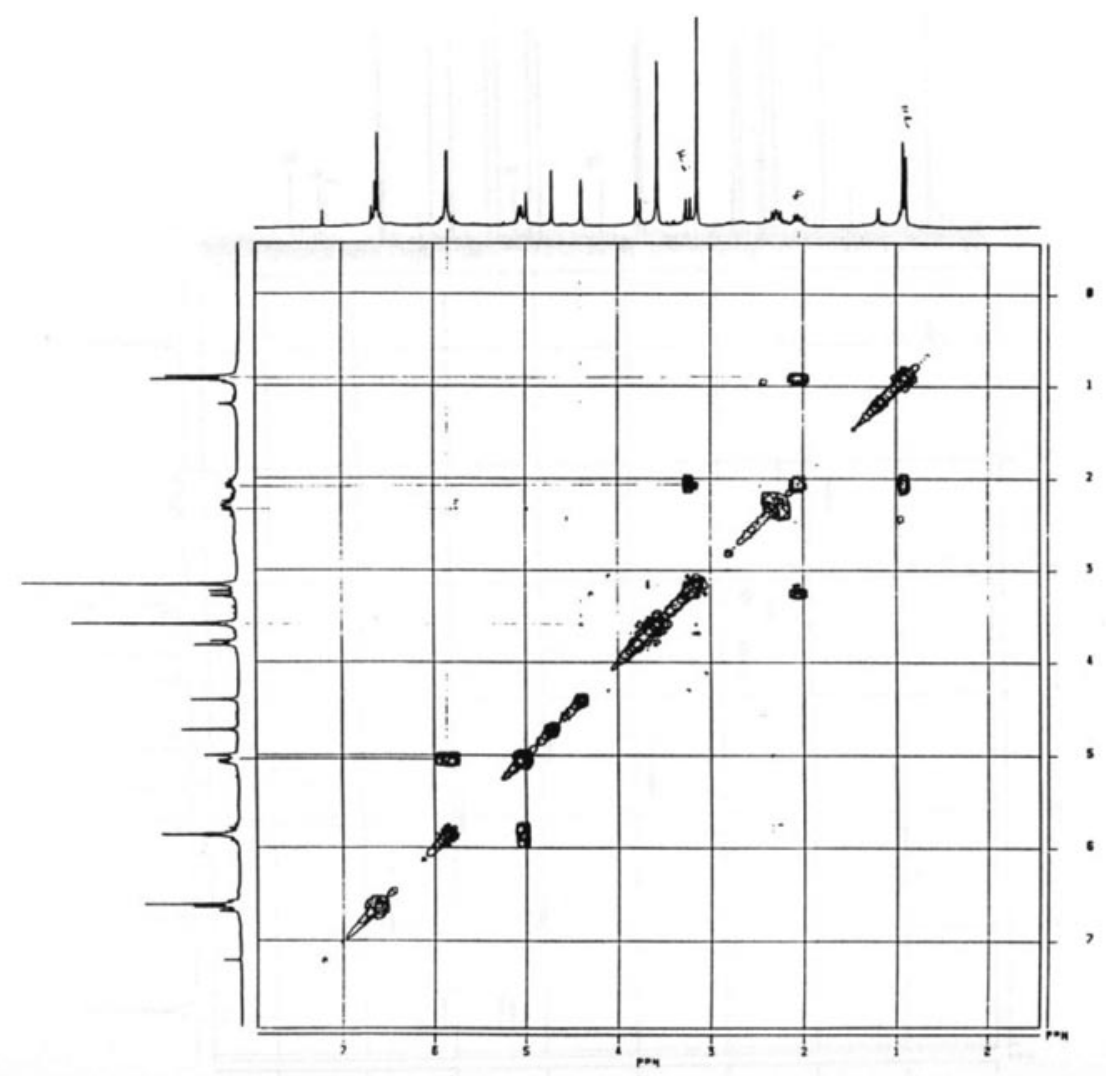

Figure S48. COSY ${ }^{13} \mathrm{C}$ NMR spectrum of $\mathbf{5 b}\left(50 \mathrm{MHz}, \mathrm{CDCl}_{3}\right)$.

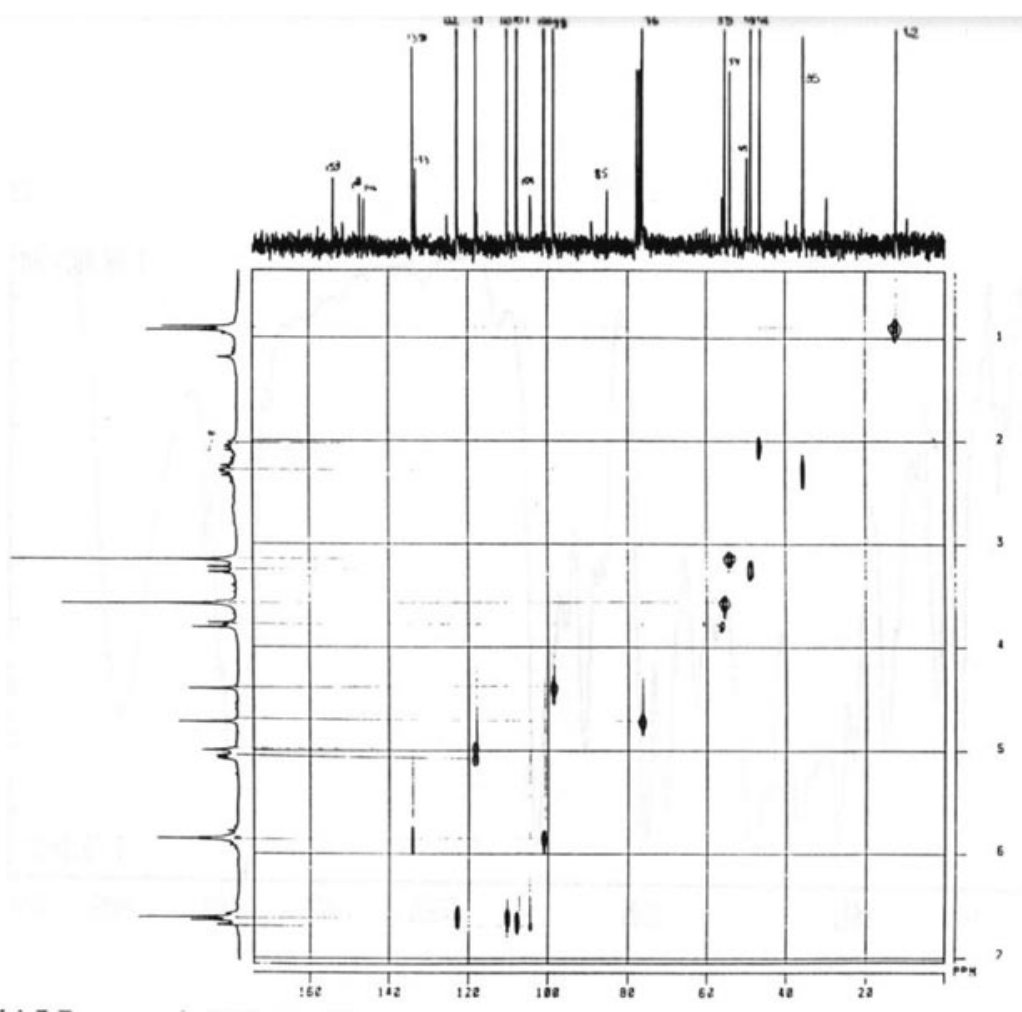

Figure S49. HETCOR ${ }^{13} \mathrm{C}$ NMR spectrum of $\mathbf{5 b}\left(50 \mathrm{MHz}, \mathrm{CDCl}_{3}\right)$. 


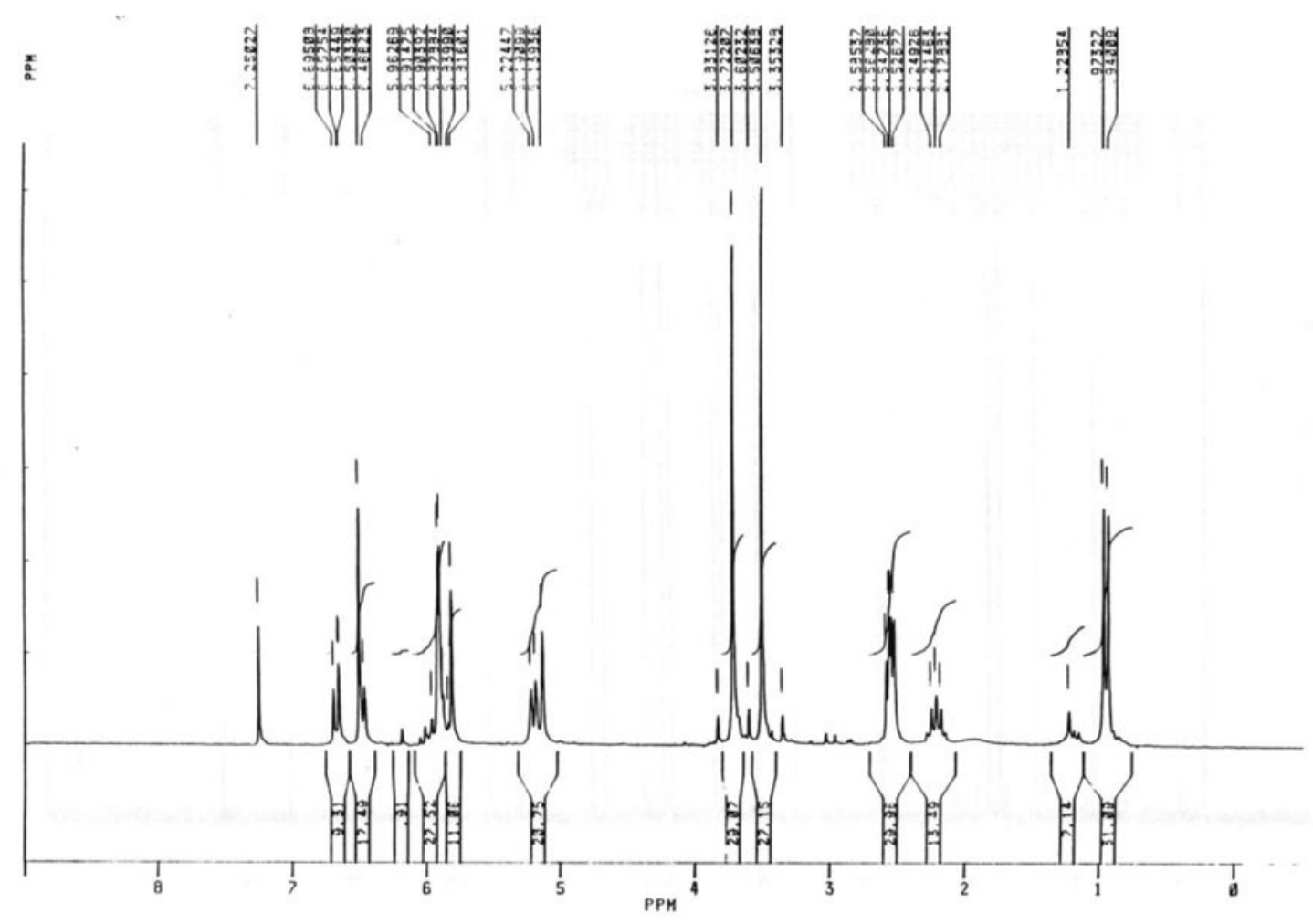

Figure S50. ${ }^{1} \mathrm{H}$ NMR spectrum of $\mathbf{5 c}\left(200 \mathrm{MHz}, \mathrm{CDCl}_{3}\right)$.

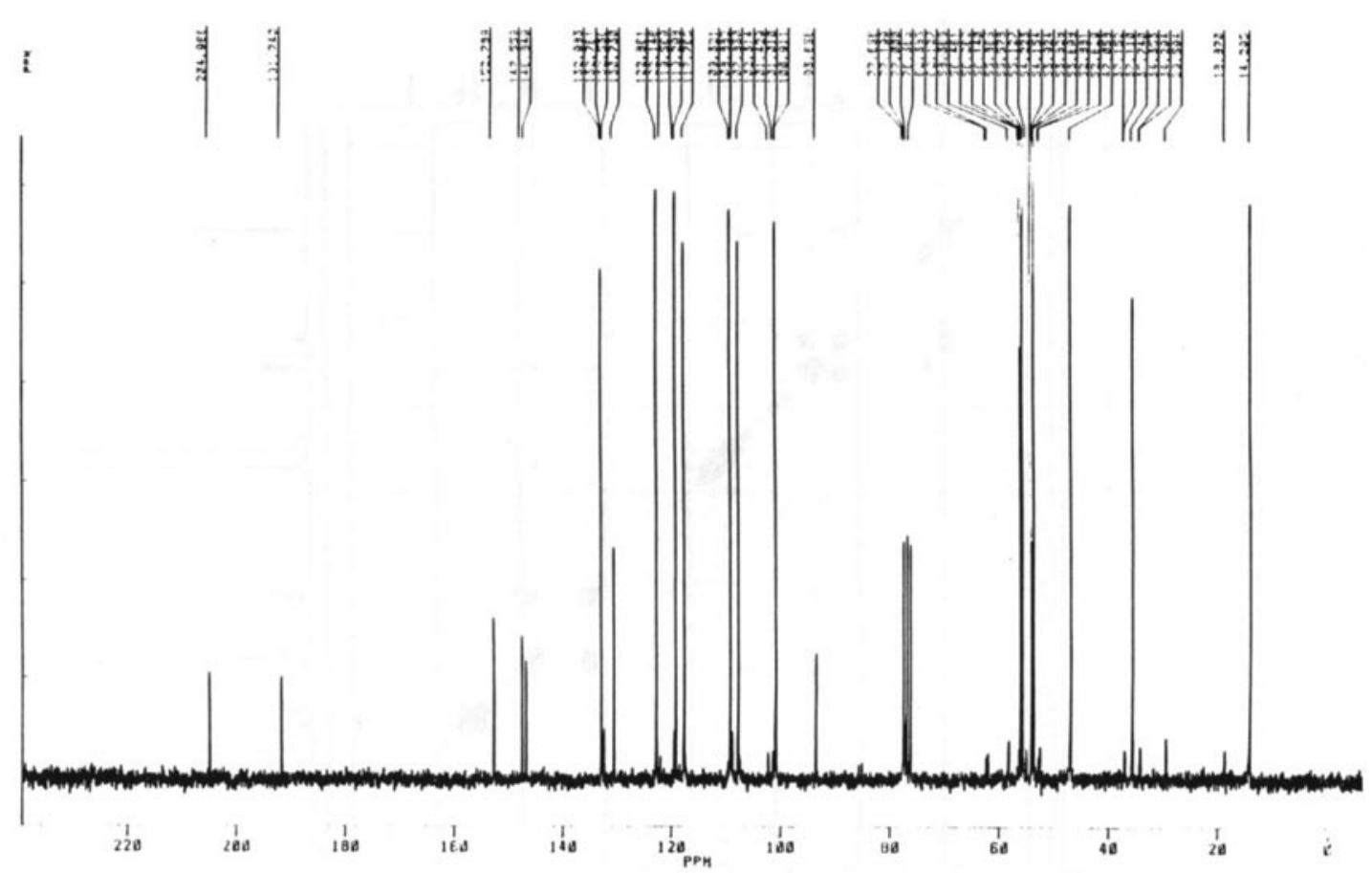

Figure S51. ${ }^{13} \mathrm{C}$ NMR spectrum of $\mathbf{5 c}\left(50 \mathrm{MHz}, \mathrm{CDCl}_{3}\right)$. 


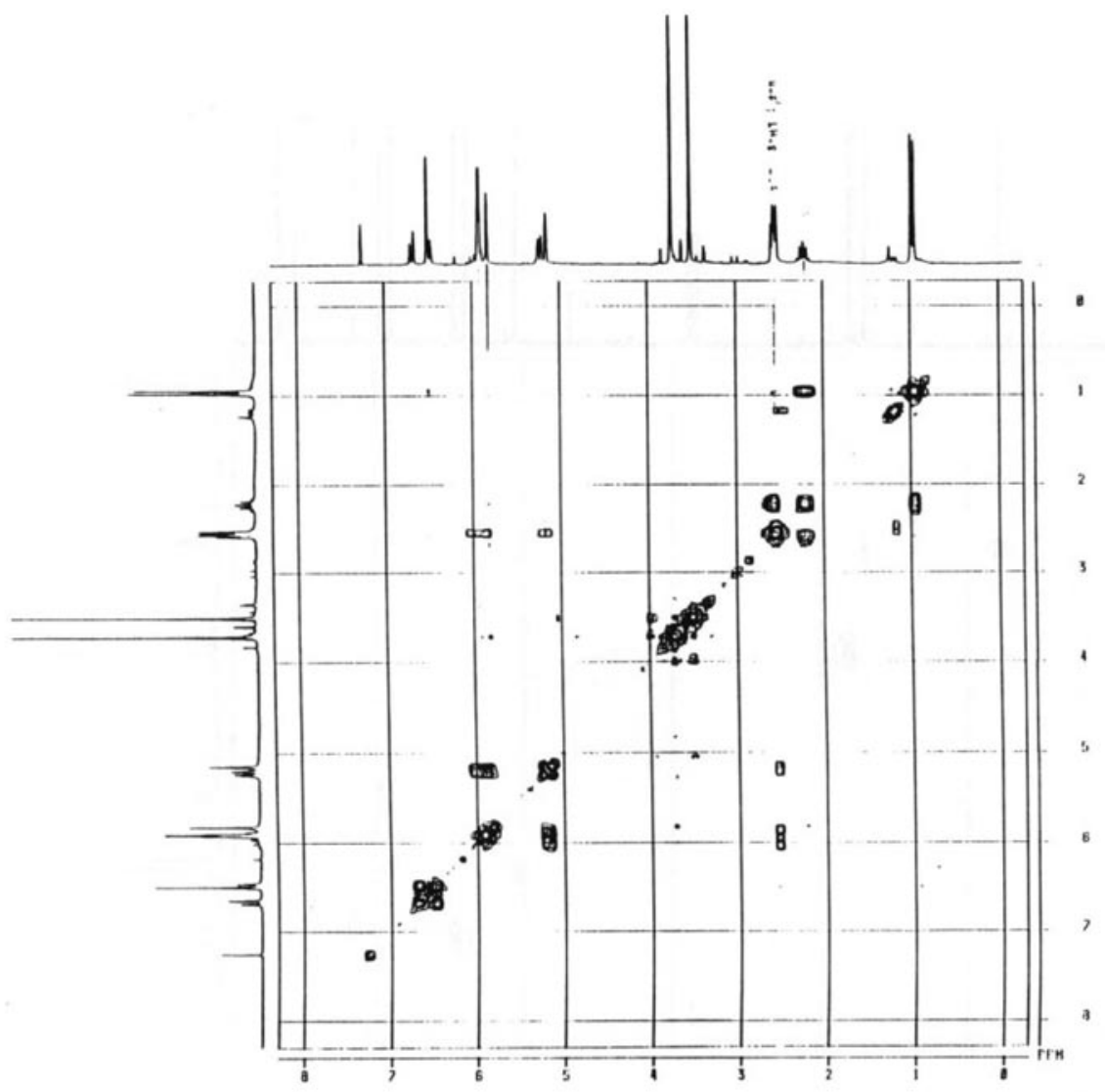

Figure S52. COSY ${ }^{13} \mathrm{C}$ NMR spectrum of $\mathbf{5 c}\left(50 \mathrm{MHz}, \mathrm{CDCl}_{3}\right)$.

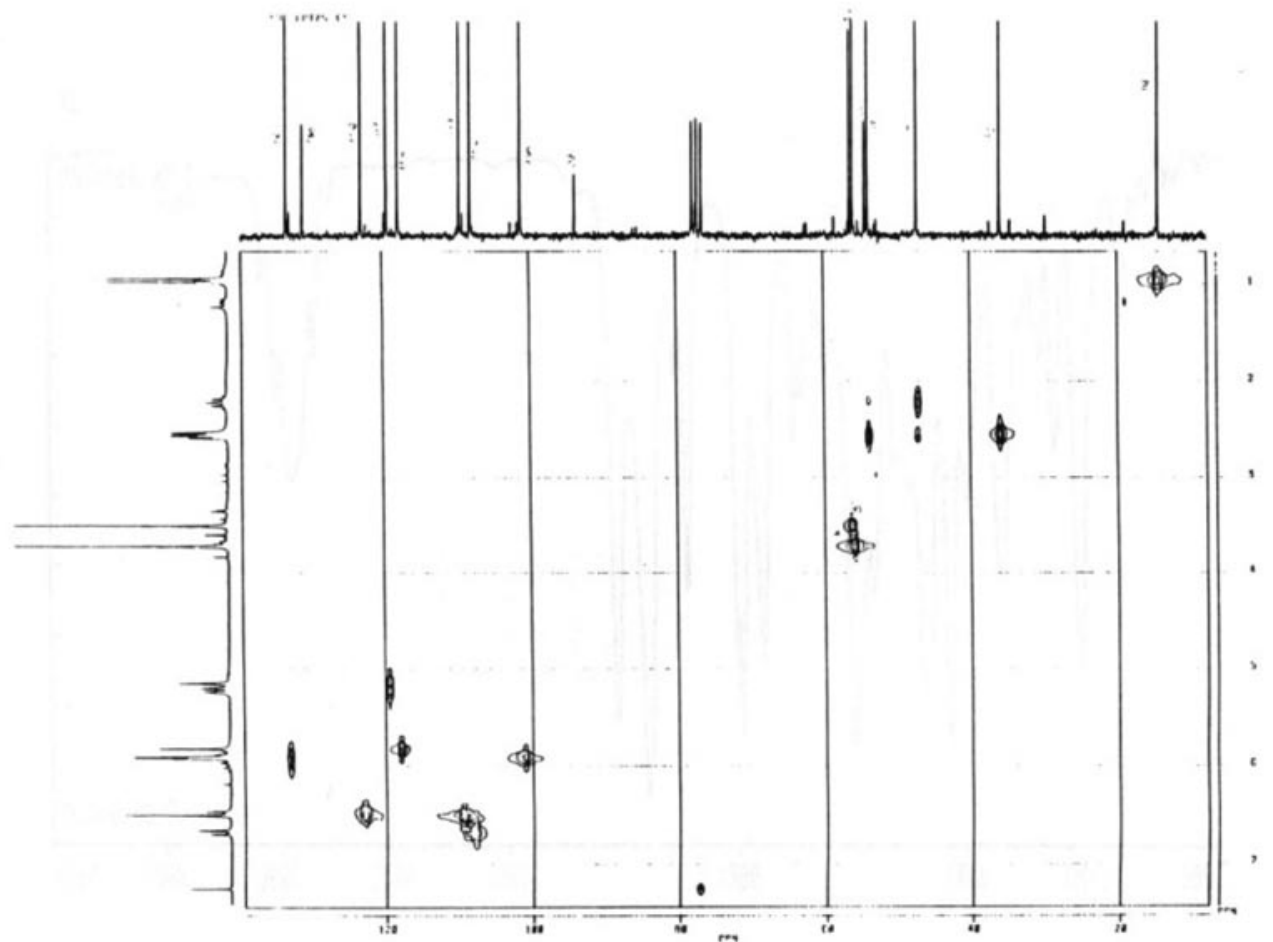

Figure S53. $\mathrm{HETCOR}{ }^{13} \mathrm{C}$ NMR spectrum of $\mathbf{5 c}\left(50 \mathrm{MHz}, \mathrm{CDCl}_{3}\right)$. 


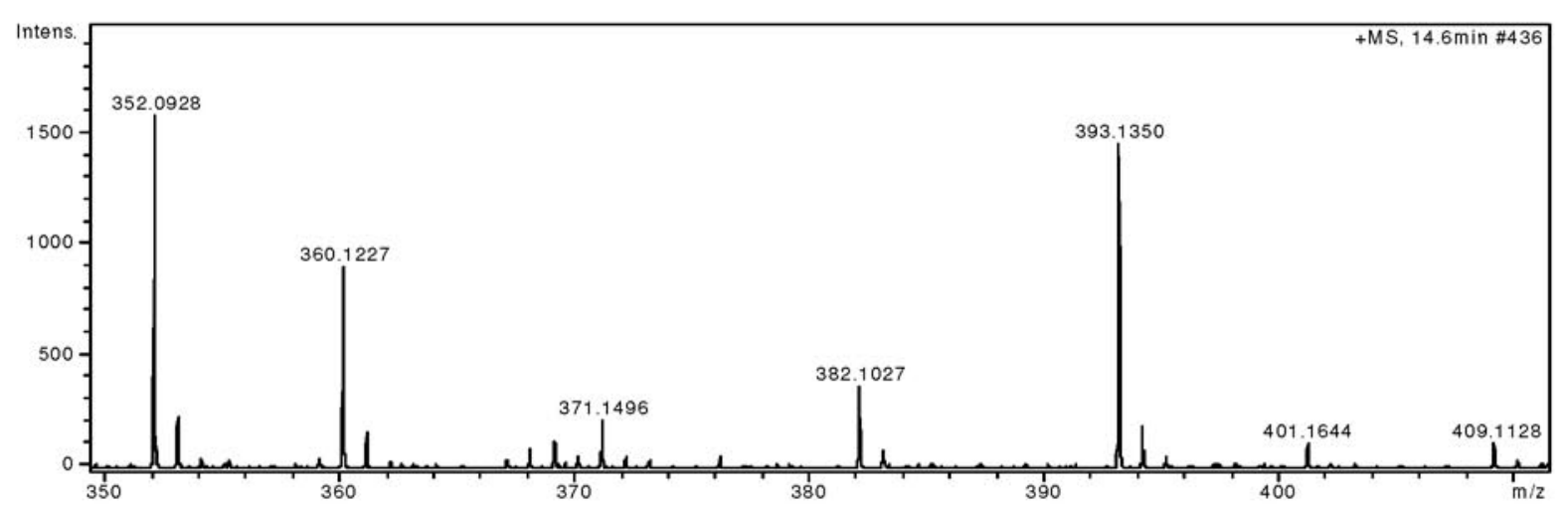

Figure S54. HRESIMS spectrum of 5c.

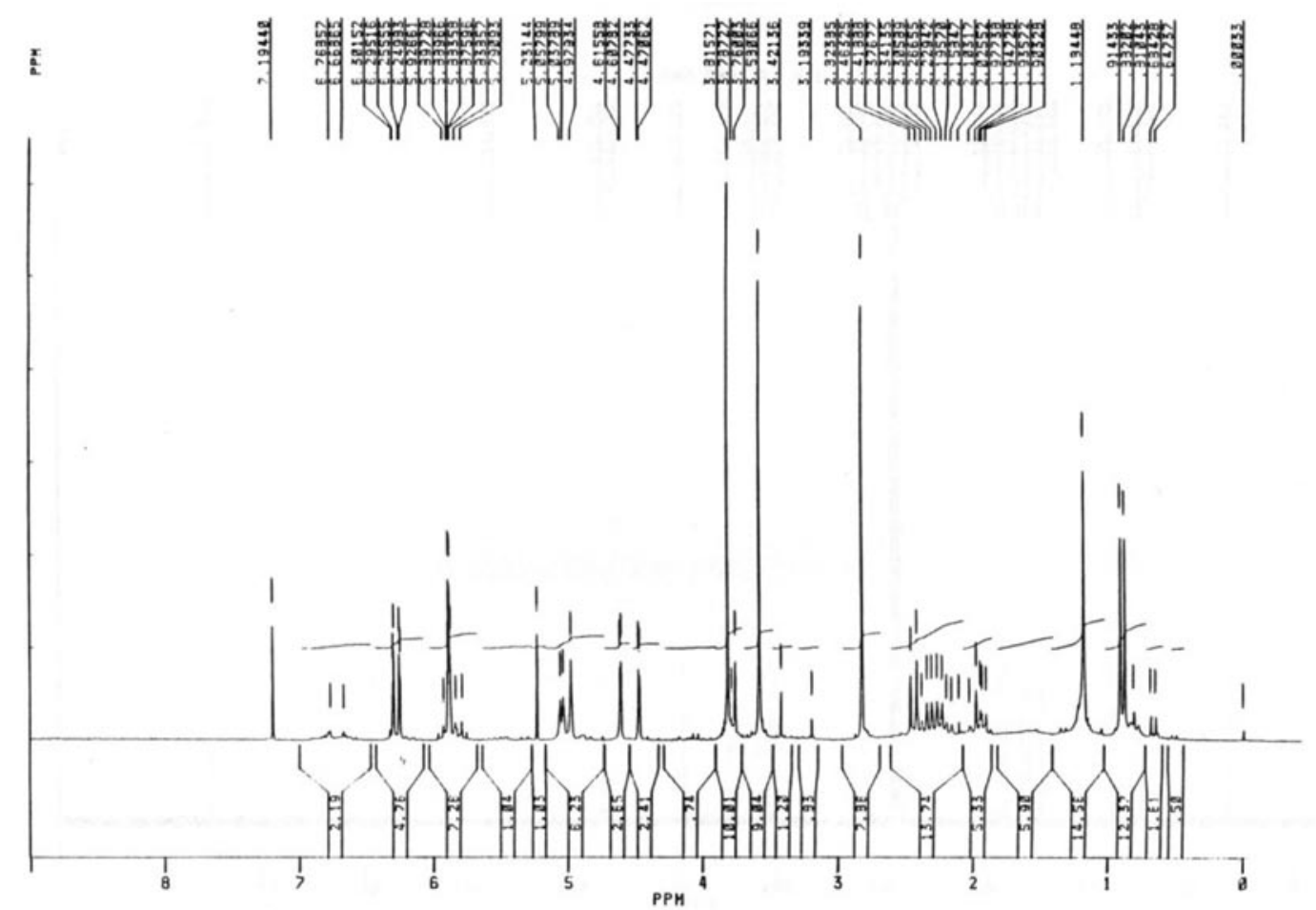

Figure S55. ${ }^{1} \mathrm{H}$ NMR spectrum of $\mathbf{5 d}\left(200 \mathrm{MHz}, \mathrm{CDCl}_{3}\right)$. 


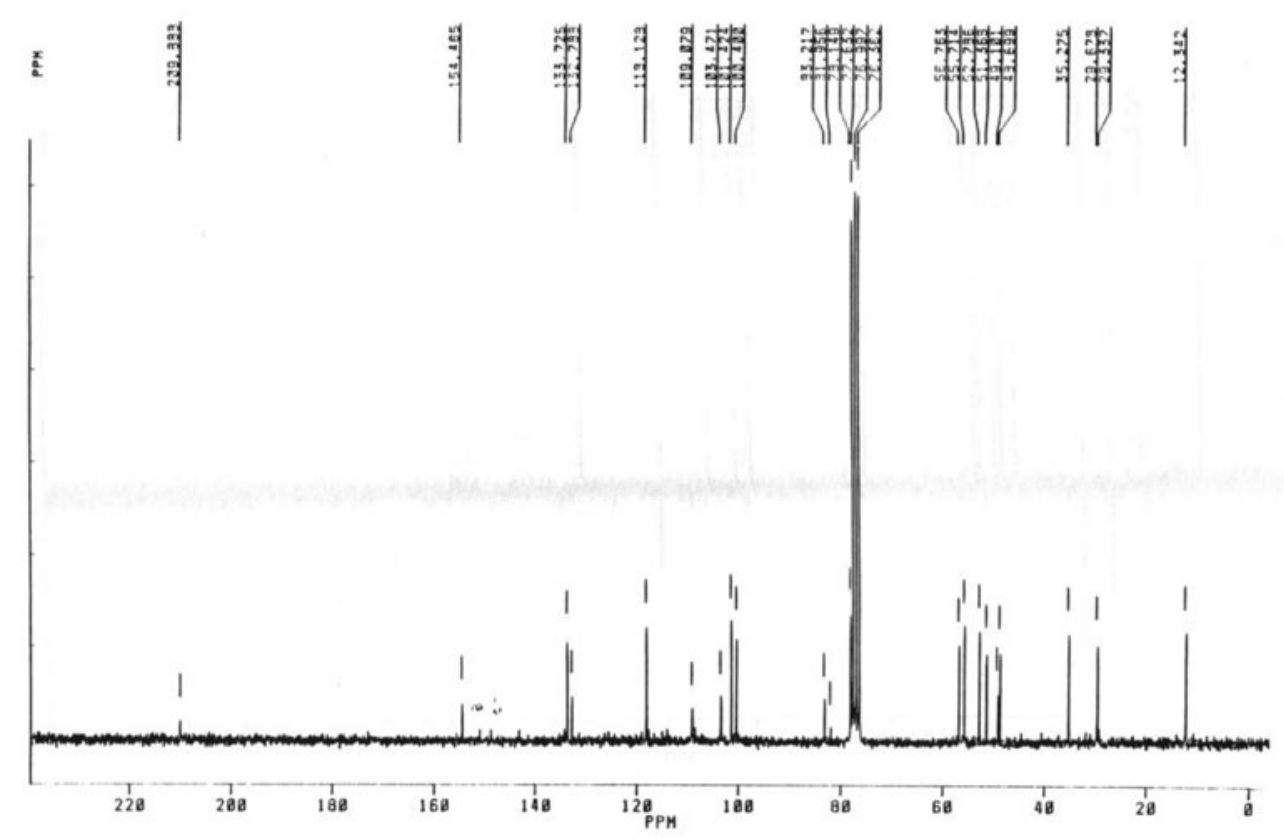

Figure S56. ${ }^{13} \mathrm{C}$ NMR spectrum of $\mathbf{5 d}\left(50 \mathrm{MHz}, \mathrm{CDCl}_{3}\right)$.

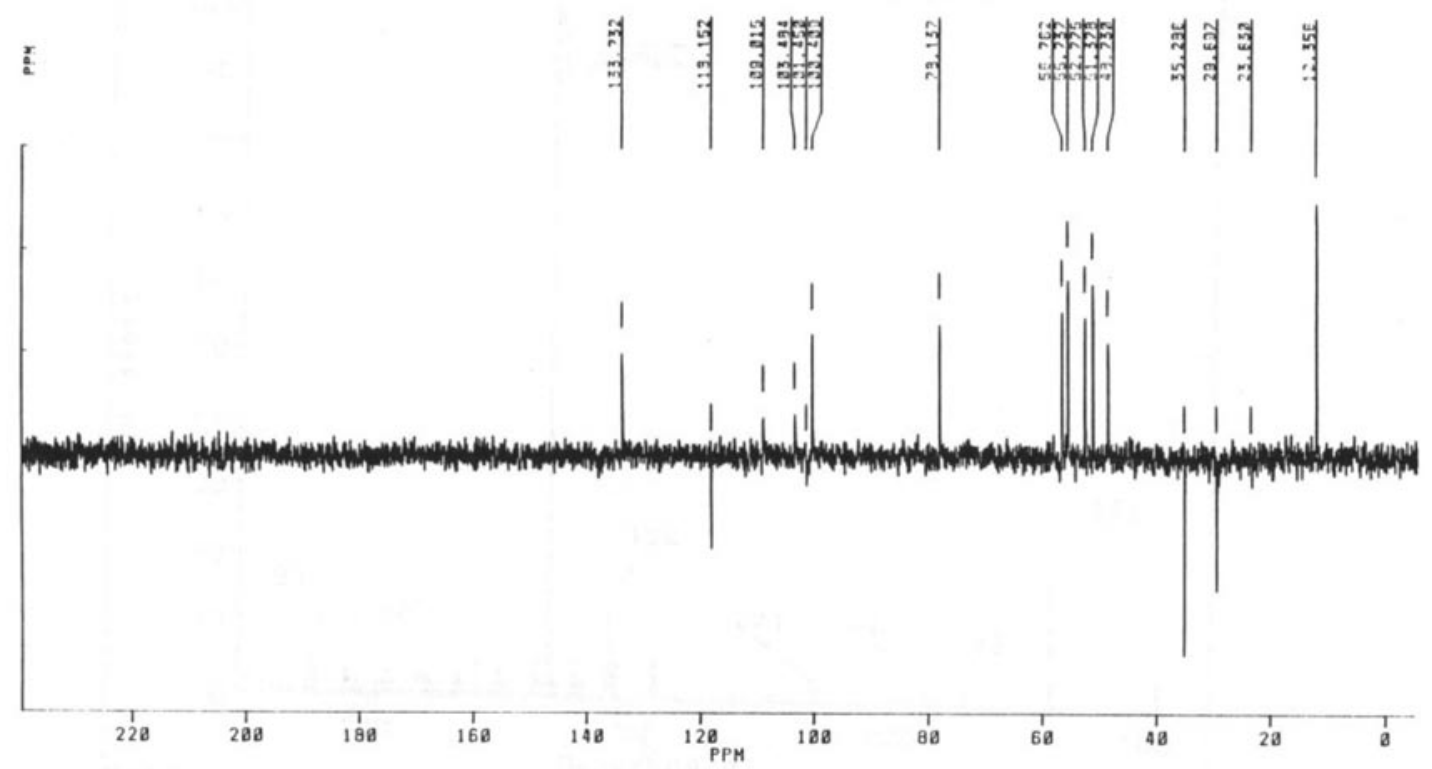

Figure S57. DEPT $135{ }^{13} \mathrm{C}$ NMR spectrum of $5 \mathbf{d}\left(50 \mathrm{MHz}, \mathrm{CDCl}_{3}\right)$. 


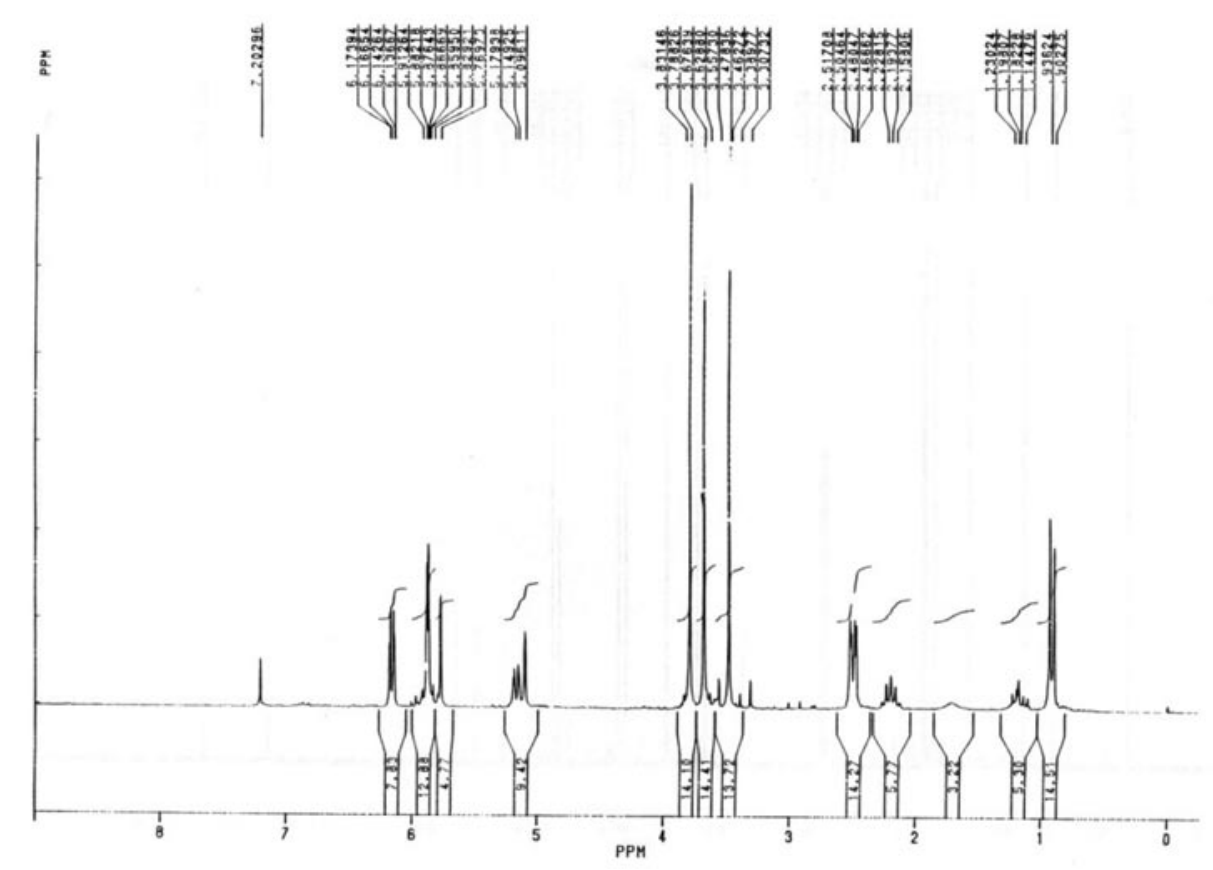

Figure S58. ${ }^{1} \mathrm{H}$ NMR spectrum of $\mathbf{5 e}\left(200 \mathrm{MHz}, \mathrm{CDCl}_{3}\right)$.

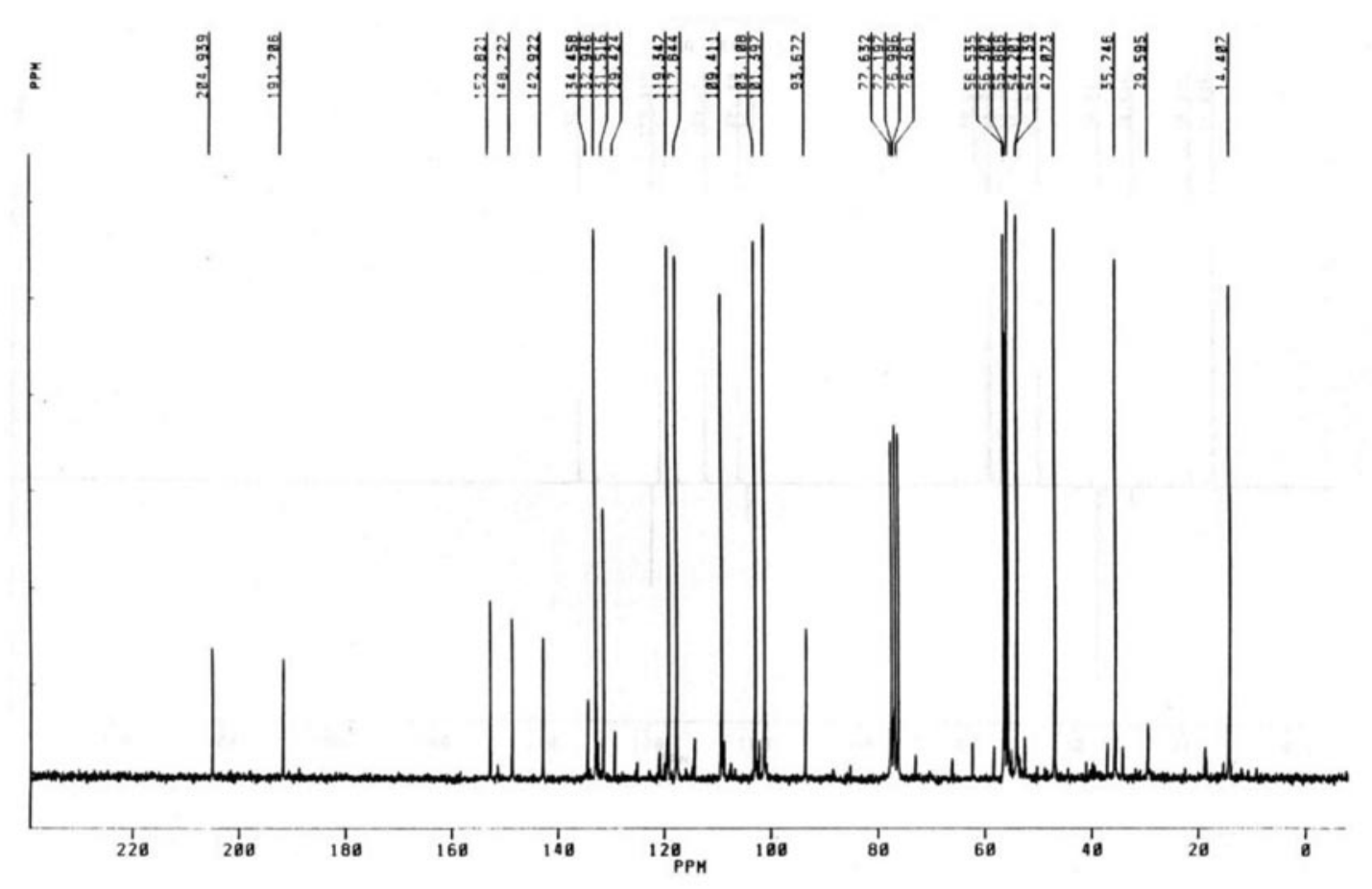

Figure S59. ${ }^{13} \mathrm{C}$ NMR spectrum of $\mathbf{5 e}\left(50 \mathrm{MHz}, \mathrm{CDCl}_{3}\right)$. 


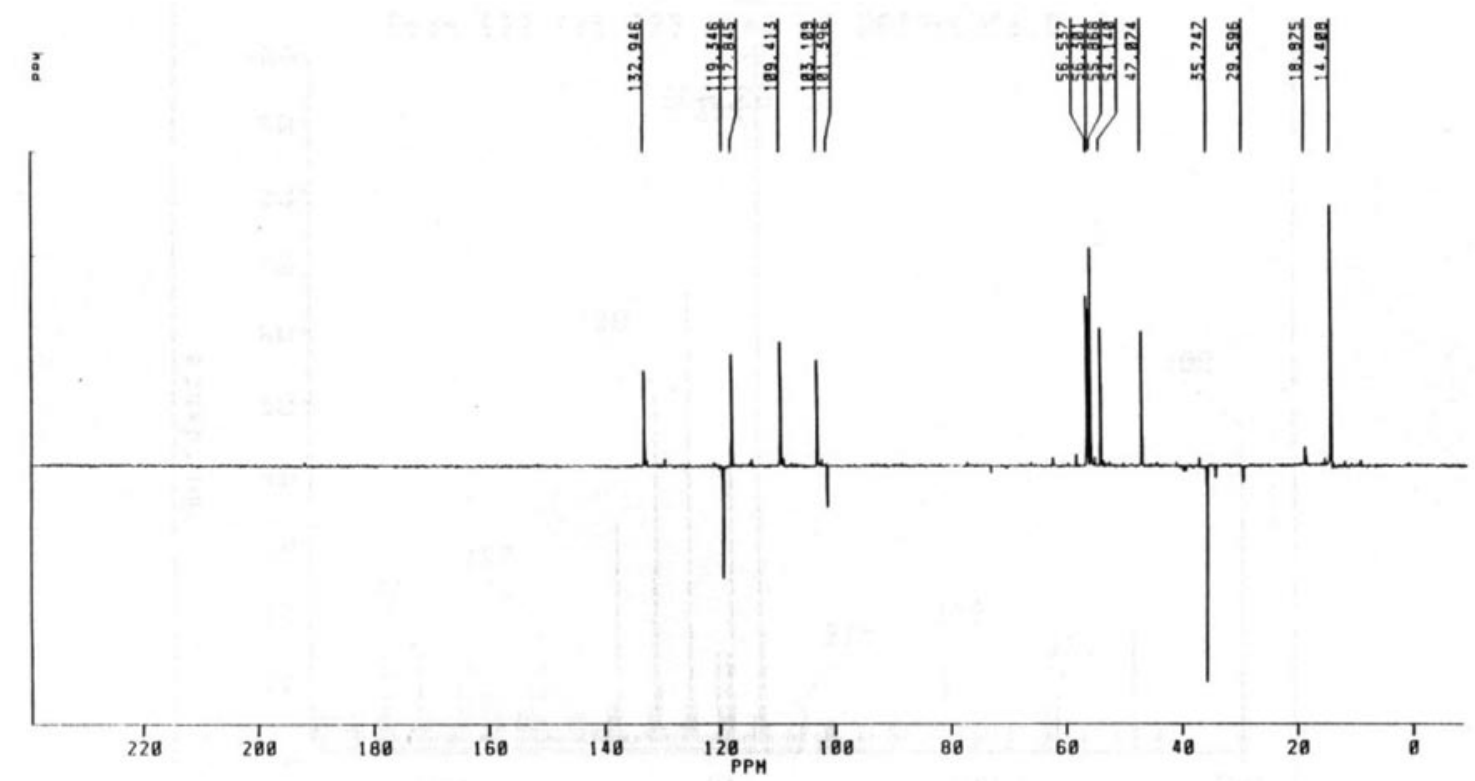

Figure S60. DEPT $135{ }^{13} \mathrm{C}$ NMR spectrum of $5 \mathbf{e}\left(50 \mathrm{MHz}, \mathrm{CDCl}_{3}\right)$.

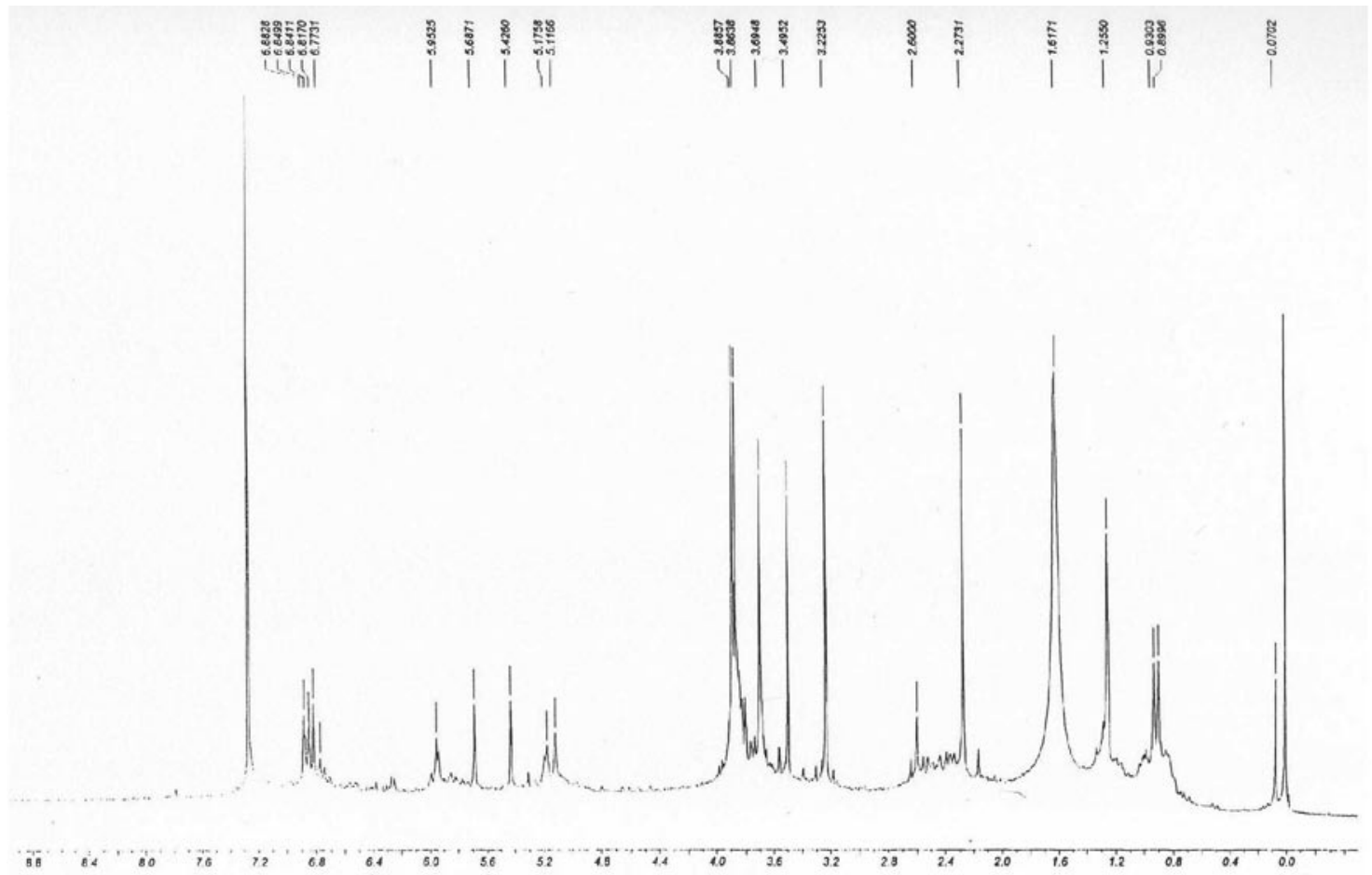

Figure S61. ${ }^{1} \mathrm{H}$ NMR spectrum of $6\left(200 \mathrm{MHz}, \mathrm{CDCl}_{3}\right)$. 


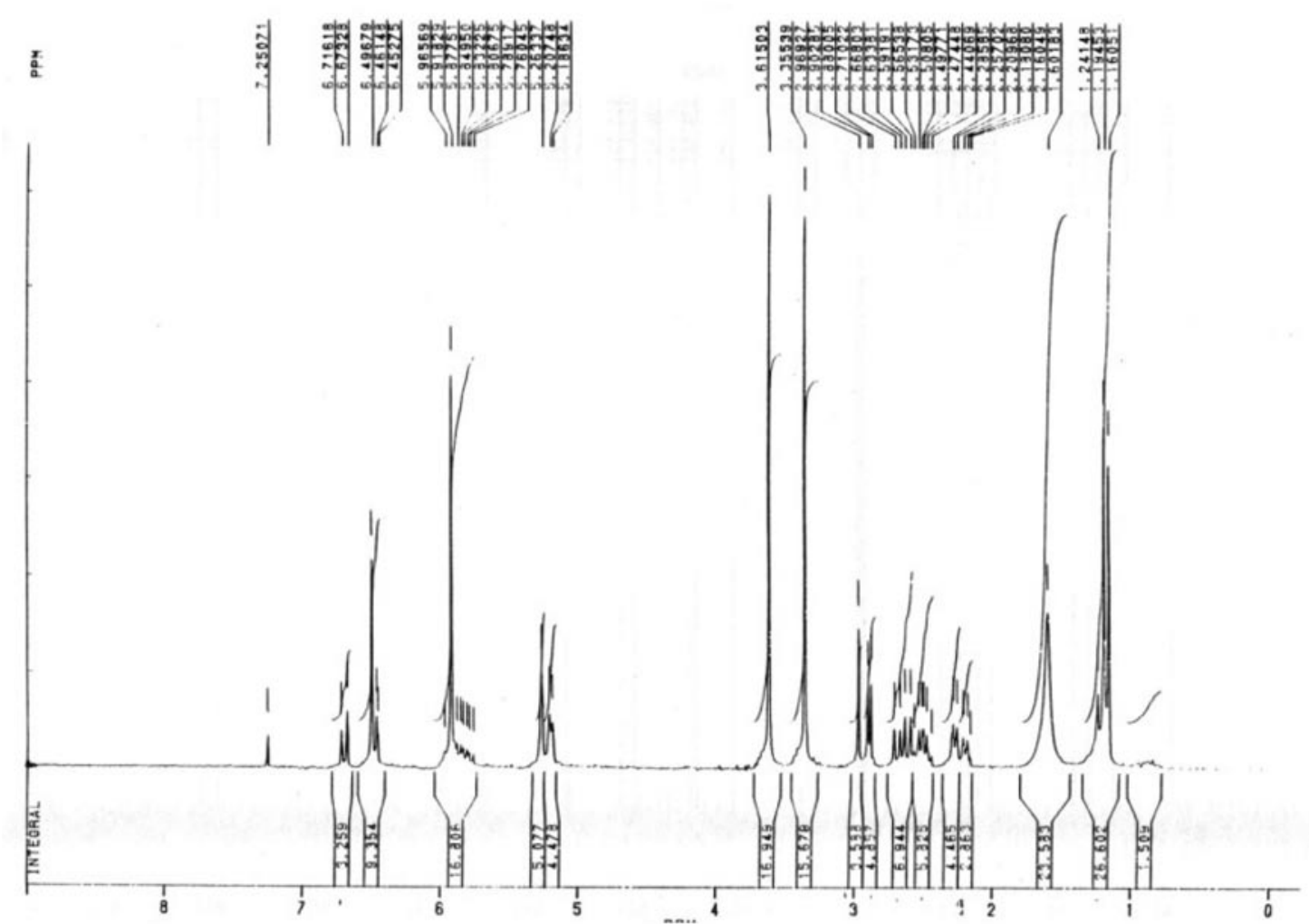

Figure S62. ${ }^{1} \mathrm{H}$ NMR spectrum of $7 \mathbf{a}\left(200 \mathrm{MHz}, \mathrm{CDCl}_{3}\right)$.

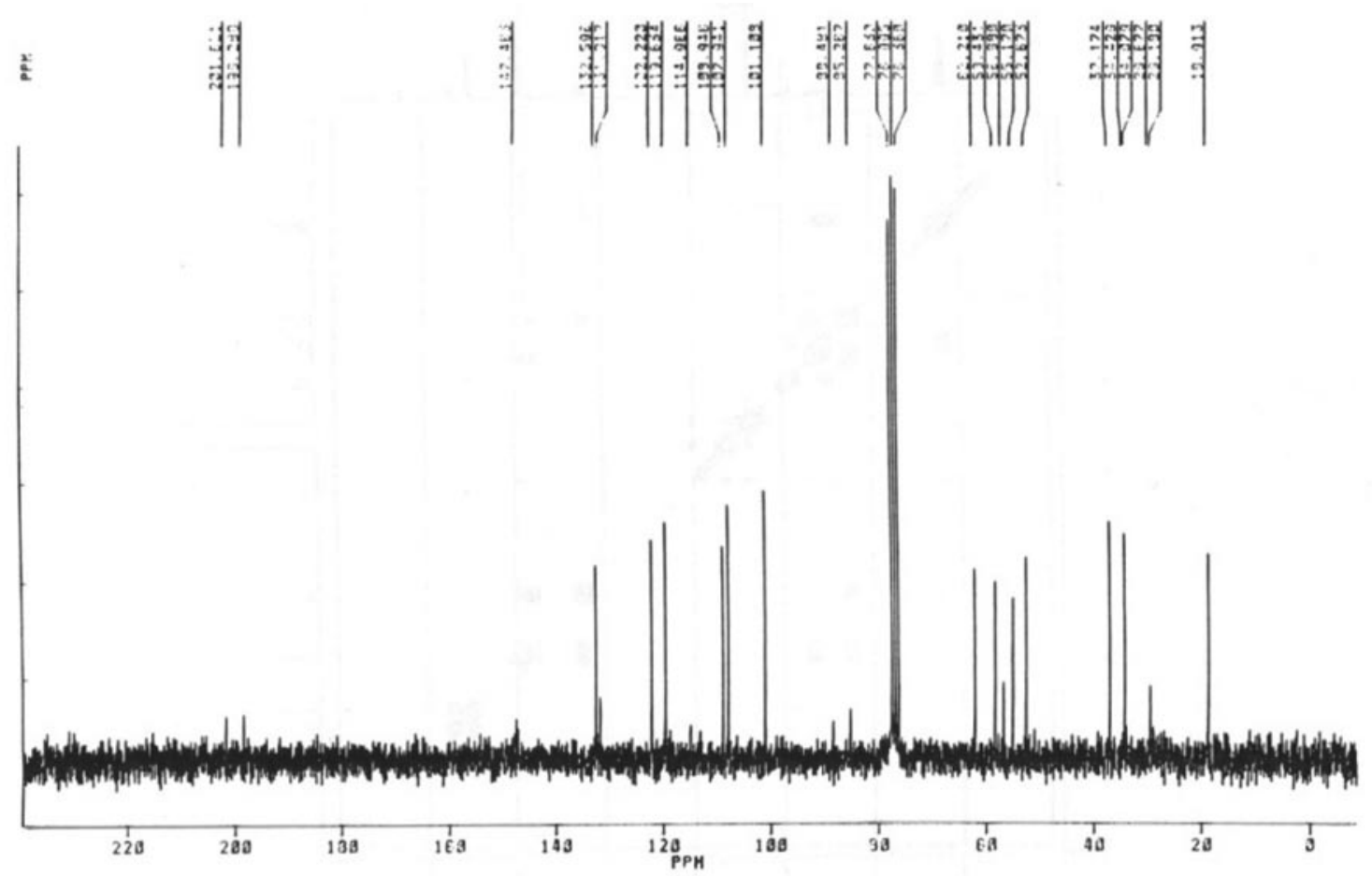

Figure S63. ${ }^{13} \mathrm{C}$ NMR spectrum of $\mathbf{7 a}\left(50 \mathrm{MHz}, \mathrm{CDCl}_{3}\right)$. 


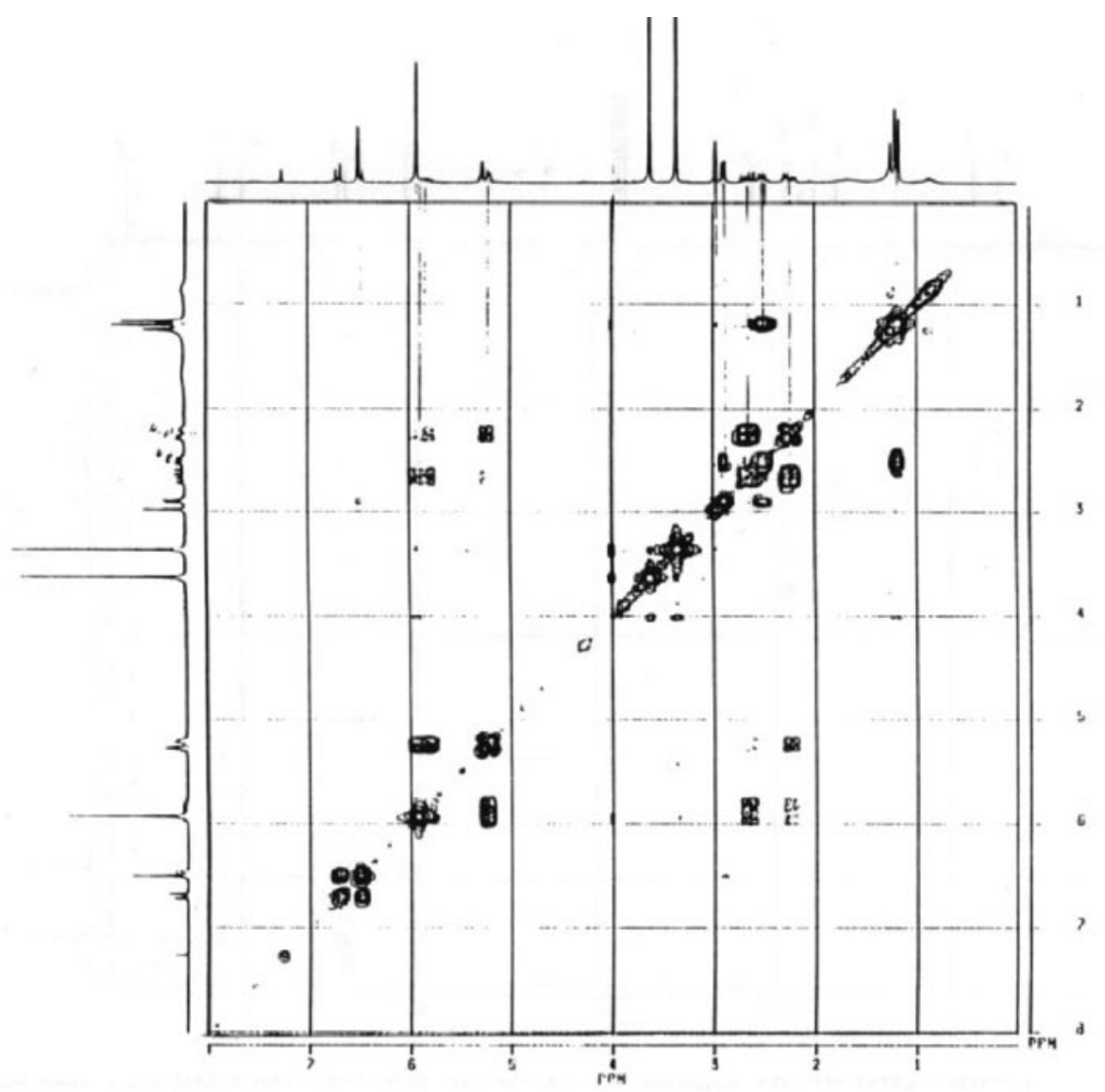

Figure S64. COSY ${ }^{13} \mathrm{C}$ NMR spectrum of $7 \mathbf{a}\left(50 \mathrm{MHz}, \mathrm{CDCl}_{3}\right)$.

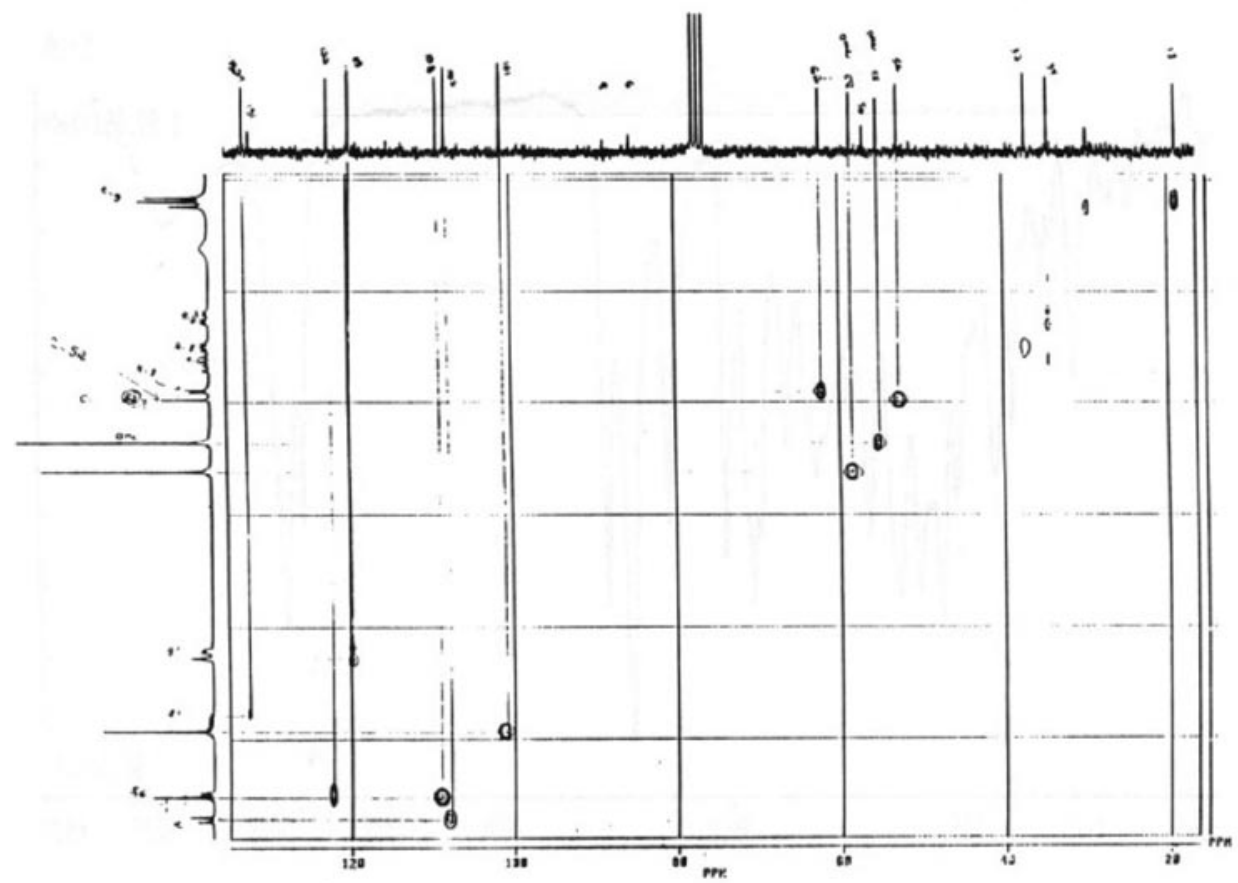

Figure S65. HETCOR ${ }^{13} \mathrm{C}$ NMR spectrum of $7 \mathbf{a}\left(50 \mathrm{MHz}, \mathrm{CDCl}_{3}\right)$. 


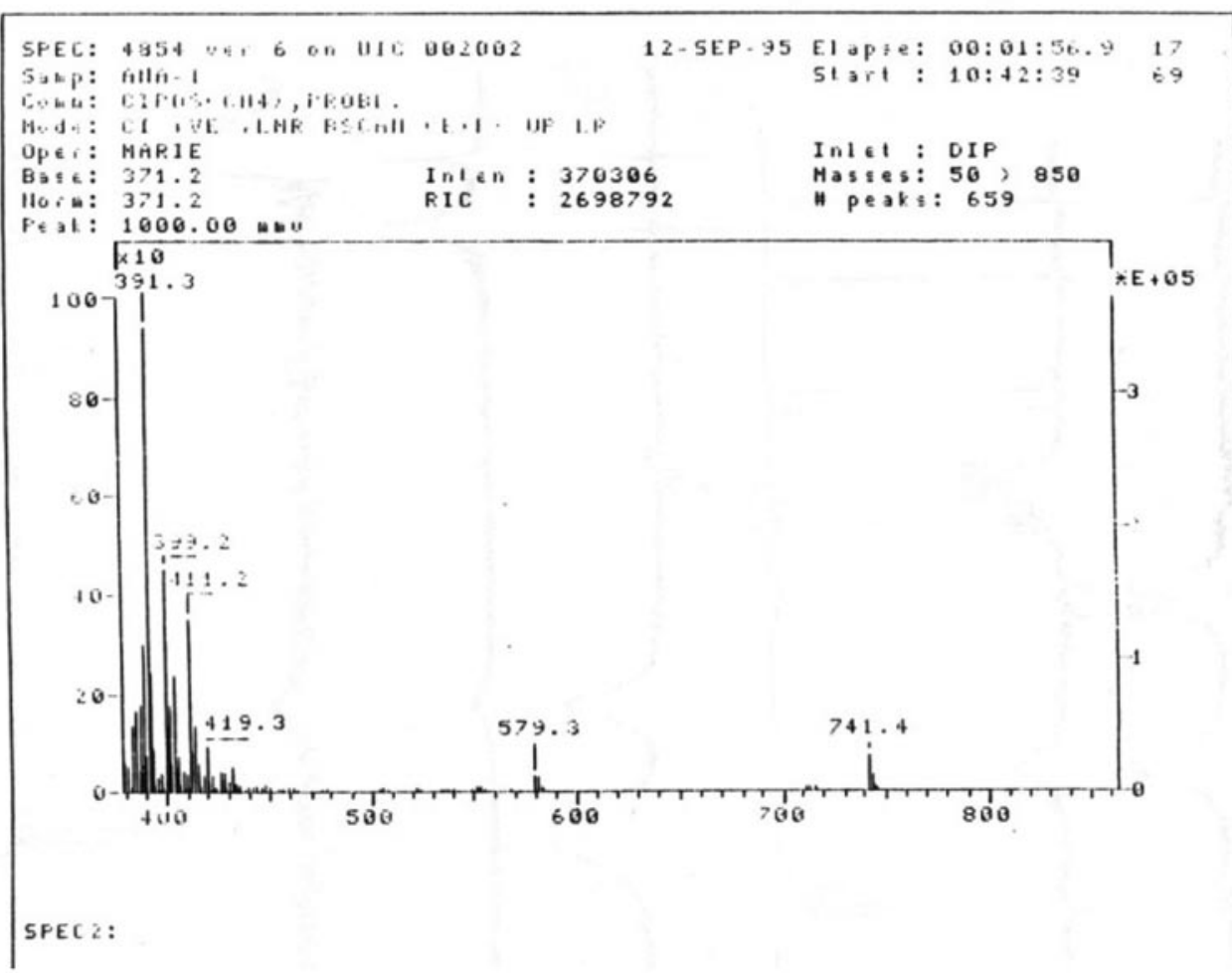

Figure S66. EIMS spectrum of $7 \mathbf{a}(70 \mathrm{eV})$.

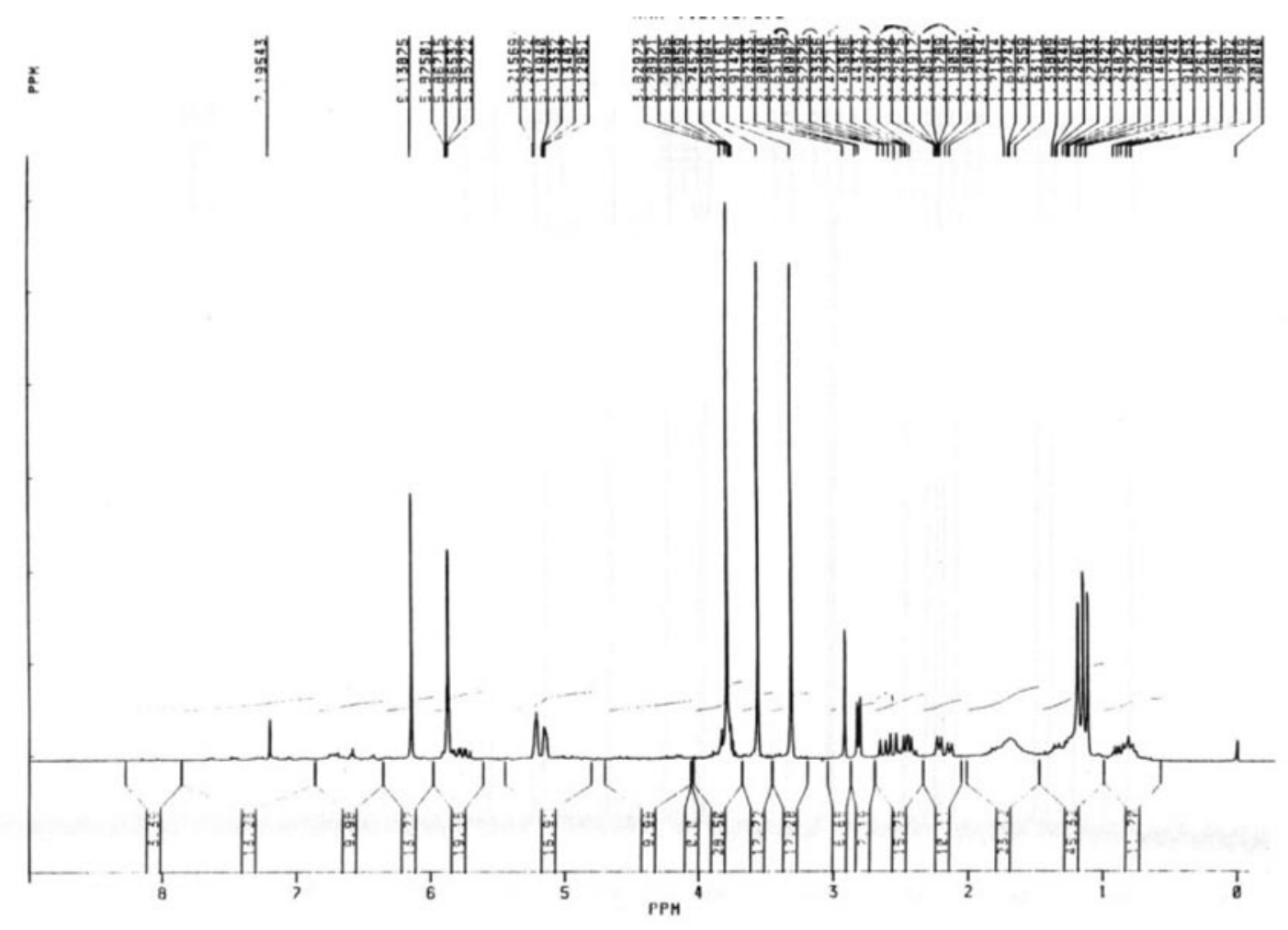

Figure S67. ${ }^{1} \mathrm{H}$ NMR spectrum of $7 \mathbf{a}\left(200 \mathrm{MHz}, \mathrm{CDCl}_{3}\right)$. 

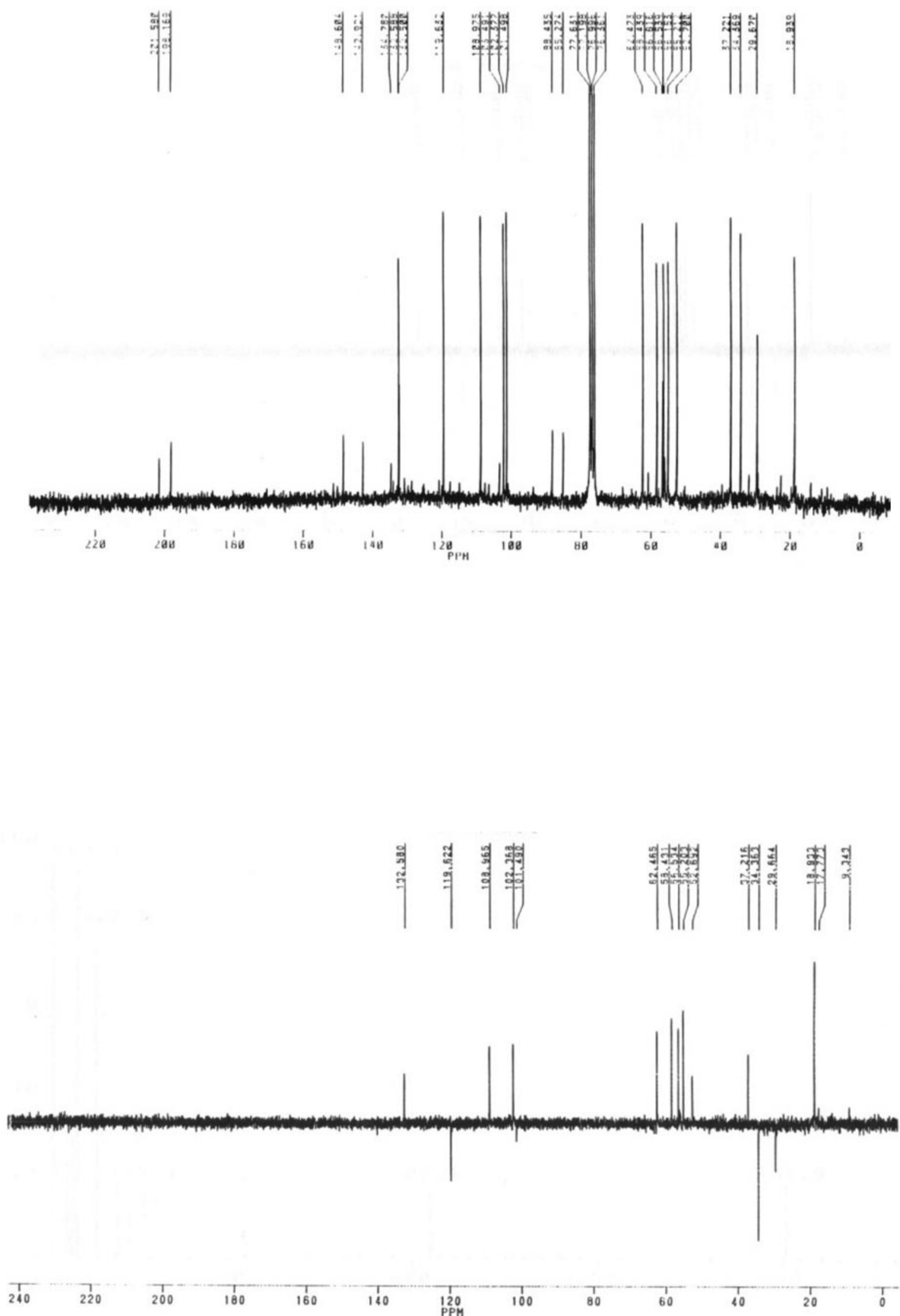

Figure S69. DEPT $135{ }^{13} \mathrm{C}$ NMR spectrum of $\mathbf{5 e}\left(50 \mathrm{MHz}, \mathrm{CDCl}_{3}\right)$. 


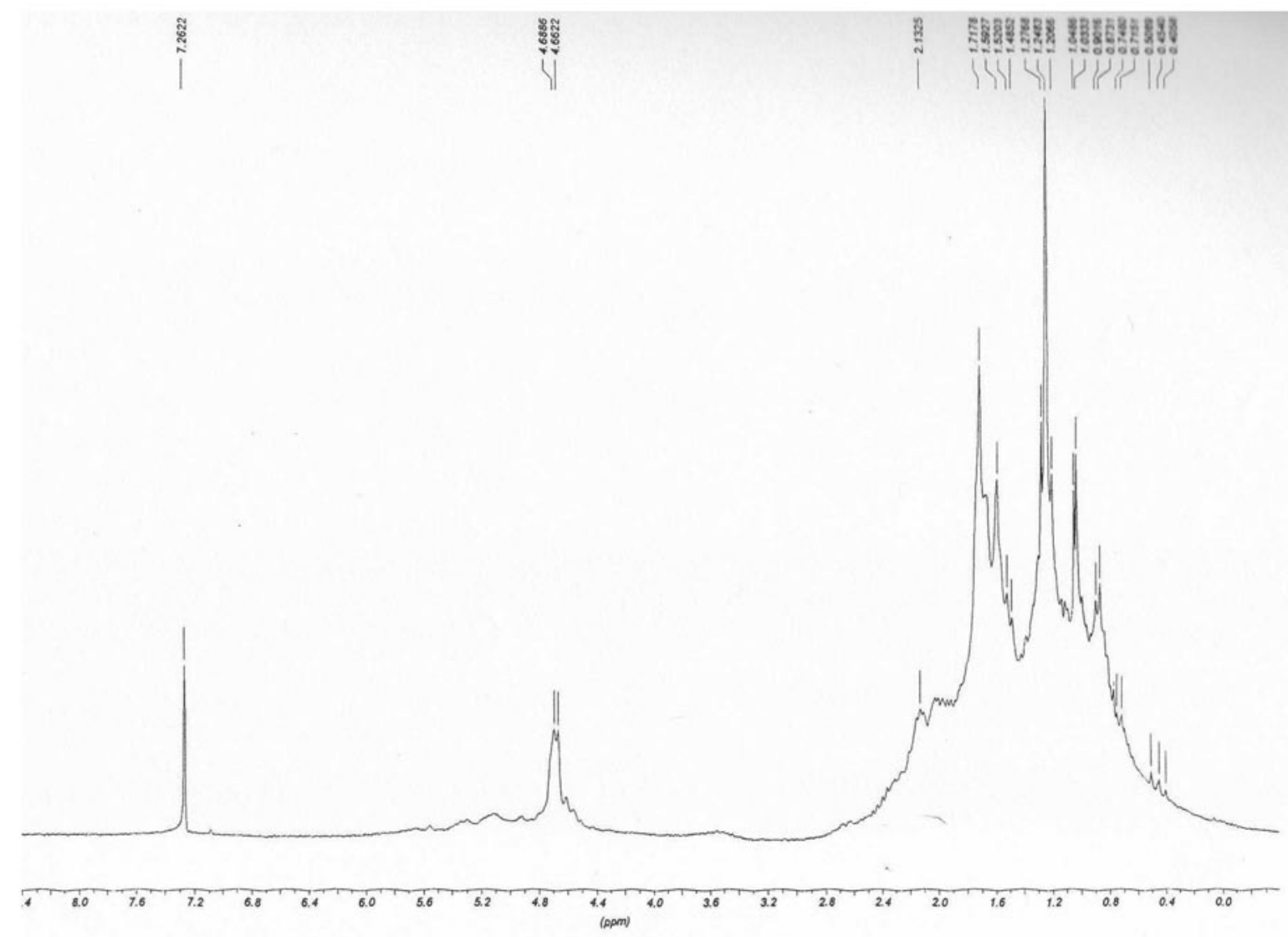

Figure S70. ${ }^{1} \mathrm{H}$ NMR spectrum of $8\left(200 \mathrm{MHz}, \mathrm{CDCl}_{3}\right)$.

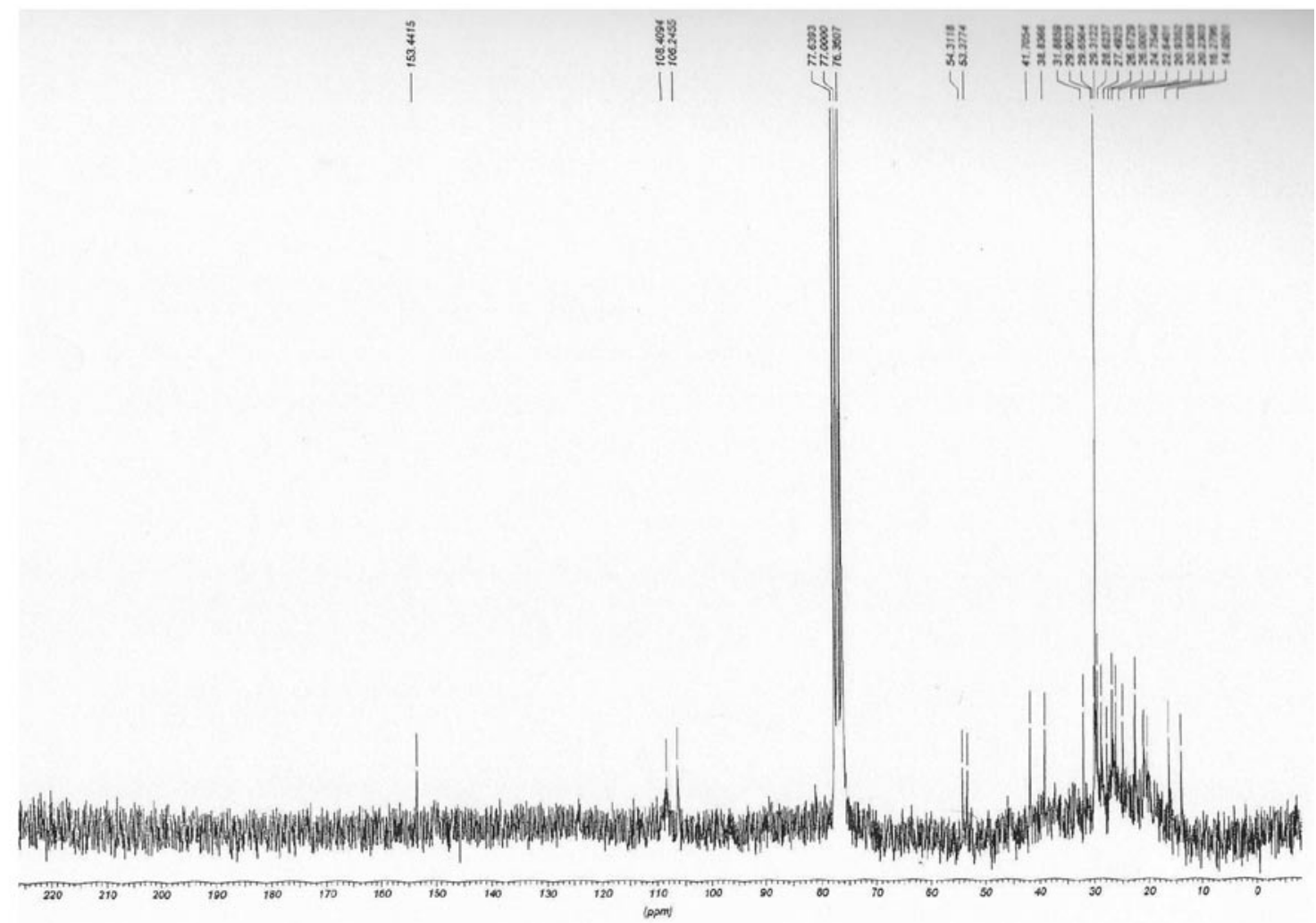

Figure S71. ${ }^{13} \mathrm{C}$ NMR spectrum of $8\left(50 \mathrm{MHz}, \mathrm{CDCl}_{3}\right)$. 

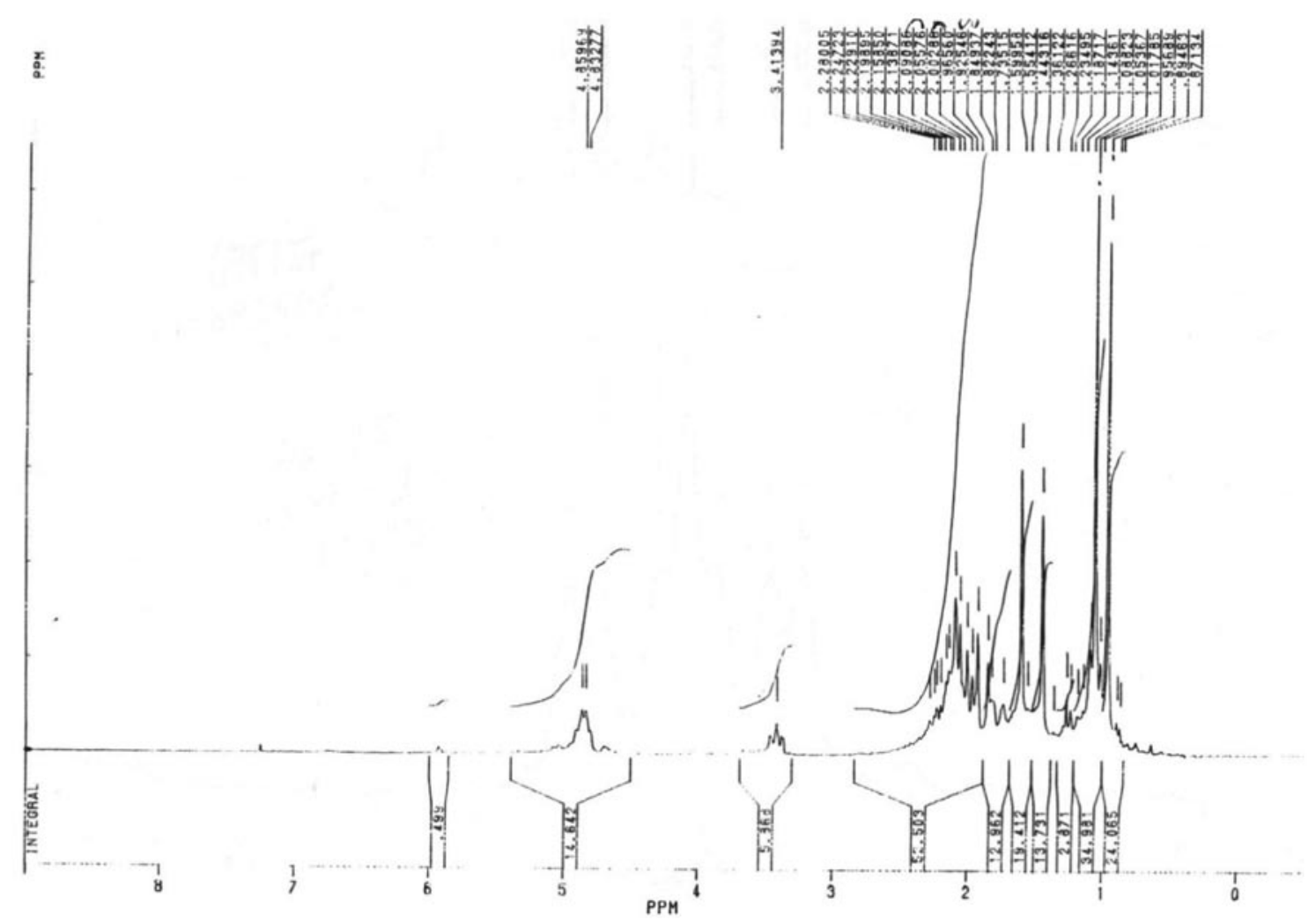

Figure S72. ' $\mathrm{H}$ NMR spectrum of $9\left(200 \mathrm{MHz}, \mathrm{CDCl}_{3}\right)$.

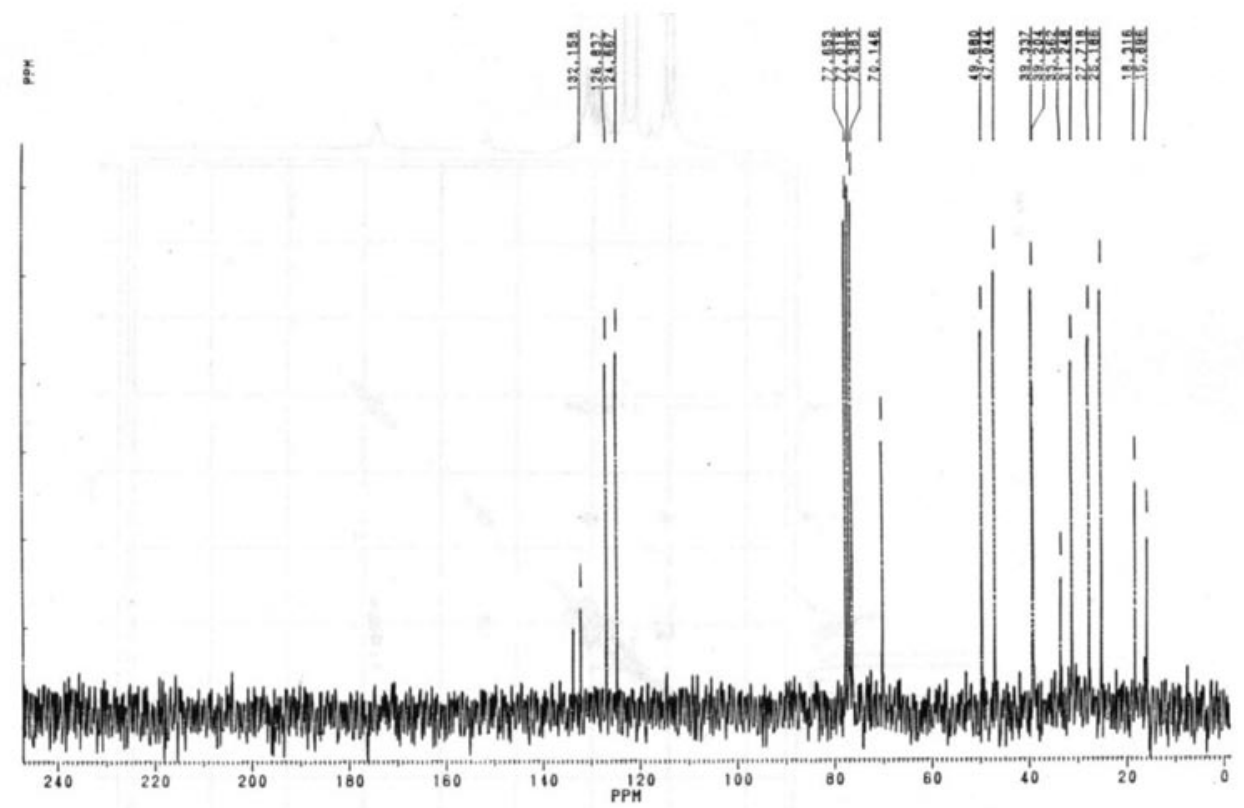

Figure S73. ${ }^{13} \mathrm{C}$ NMR spectrum of $9\left(50 \mathrm{MHz}, \mathrm{CDCl}_{3}\right)$. 


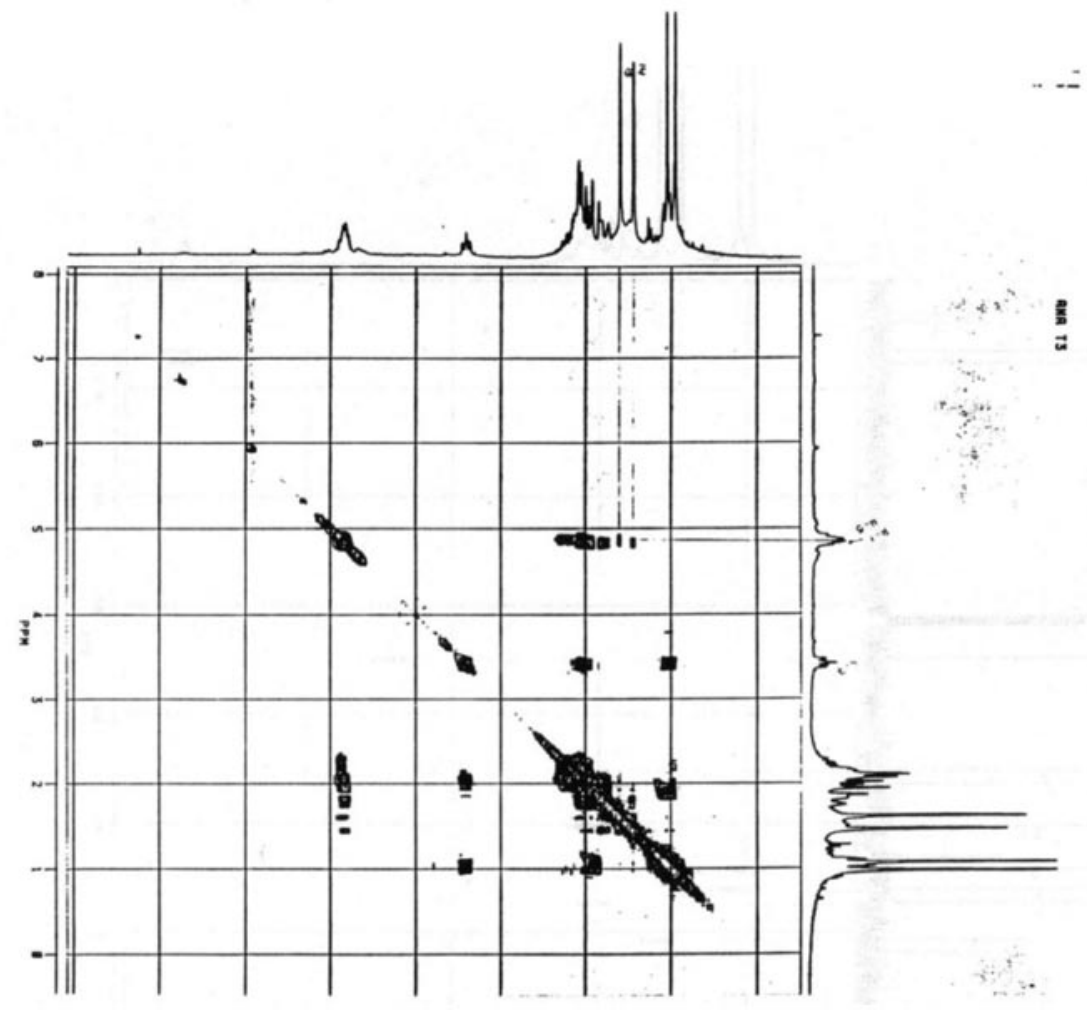

Figure S74. COSY ${ }^{13} \mathrm{C}$ NMR spectrum of $9\left(50 \mathrm{MHz}, \mathrm{CDCl}_{3}\right)$.

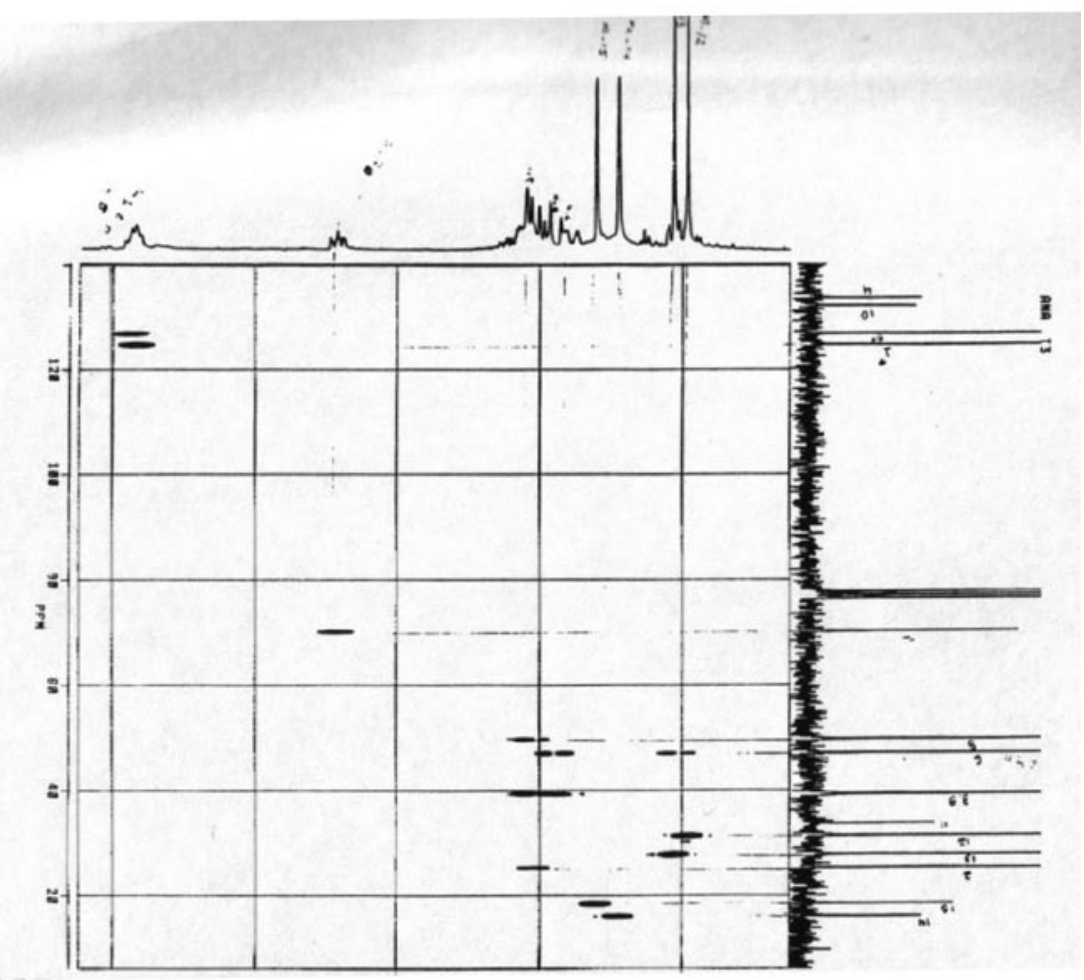

Figure S75. HETCOR ${ }^{13} \mathrm{C}$ NMR spectrum of $9\left(50 \mathrm{MHz}, \mathrm{CDCl}_{3}\right)$. 